\title{
Development of contacting material for cathode chamber in the solid oxide fuel cell
}

\author{
Tyler-Blair A. Sheppard \\ West Virginia University
}

Follow this and additional works at: https://researchrepository.wvu.edu/etd

\section{Recommended Citation}

Sheppard, Tyler-Blair A., "Development of contacting material for cathode chamber in the solid oxide fuel cell" (2007). Graduate Theses, Dissertations, and Problem Reports. 1839.

https://researchrepository.wvu.edu/etd/1839

This Thesis is protected by copyright and/or related rights. It has been brought to you by the The Research Repository @ WVU with permission from the rights-holder(s). You are free to use this Thesis in any way that is permitted by the copyright and related rights legislation that applies to your use. For other uses you must obtain permission from the rights-holder(s) directly, unless additional rights are indicated by a Creative Commons license in the record and/ or on the work itself. This Thesis has been accepted for inclusion in WVU Graduate Theses, Dissertations, and Problem Reports collection by an authorized administrator of The Research Repository @ WVU. For more information, please contact researchrepository@mail.wvu.edu. 


\title{
DEVELOPMENT OF CONTACTING MATERIAL FOR CATHODE CHAMBER IN THE SOLID OXIDE FUEL CELL
}

\author{
Tyler-Blair A. Sheppard \\ Thesis submitted to the College of Engineering and Mineral Resources \\ at West Virginia University in partial fulfillment of the \\ requirements for the degree of \\ Master of Science \\ in \\ Mechanical Engineering \\ Bruce S. Kang, Ph.D., Chair \\ Christopher Johnson, Ph.D. \\ Darran Cairns, Ph.D.
}

Department of Mechanical and Aerospace Engineering

Morgantown, West Virginia

2007

Keywords: Solid-oxide fuel cell (SOFC), Silver, LSM, Microstructure, silver diffusion, Microstructure analysis, Scanning electron microscopy 


\title{
ABSTRACT \\ DEVELOPMENT OF CONTACTING MATERIAL FOR CATHODE CHAMBER IN THE SOLID OXIDE FUEL CELL
}

\author{
Tyler-Blair A. Sheppard
}

The objective of this research was the development and long-term testing of cathode-interconnect contact materials for the solid-oxide fuel cell (SOFC). The research effort was focused on the utilization of silver and silver cerments. Silver is an attractive candidate contact material due to its high ductility, high conductivity, and low cost relative to other candidate SOFC contact materials. Furthermore, silver does not form non-conductive oxide scales in the SOFC cathode environment. One major concern about its usage is the volatility of silver at the SOFC operating temperature of around $800{ }^{\circ} \mathrm{C}$. Samples were manufactured by depositing submicron size silver particles, bimetallic composites of silver and ceria, and strontium doped lanthanum manginite (LSM) using a screen printing process and placed in a simulated cathode environment (SCE). During the course of the long term exposure, mass loss measurements and microstructure changes of the surface of the sample were made using scanning electron microscopy (SEM).

Test results revealed significant bulk silver diffusion to the LSM cathode, which may defeat the purpose of using silver or silver cerments as contact material in the SOFC cathode chamber. Non-linear mass loss rates of the both the screen printed and foil silver samples were discovered, indicating a degree of evaporative instability in the silver. The LSM coating layer proved effective in reducing the evaporation of silver, while ceria actually increased the evaporation rate of the silver. Scanning electron microscopy showed that the LSM surface was highly stable at high temperature. Excessive bulk diffusion of the silver led to the entire silver layer diffusing into the LSM coating, leaving a void in the center of the sample. 


\section{ACKNOWLEDGEMENTS}

I would like to thank my research advisor and committee chair Dr. Bruce Kang for his guidance throughout my masters program, as well as Dr. Lane Wilson. Also, I would like to thank Dr. Christopher Johnson for all his help and advice throughout my research. I would like to express thanks to both Dr. Johnson and to Dr. Darran Cairns for serving on my committee and for their help in the completion of this thesis.

To John Sakacsi, a most sincere thank you for all his guidance and help during the year we worked together.

I would also like to thank Ms. Diane Berry for all her help with my scanning electron microscopy work at NIOSH.

Finally, I would like to express my gratitude to my family and friends for always being there for me and for all there love and support over the years. 


\section{TABLE OF CONTENTS}

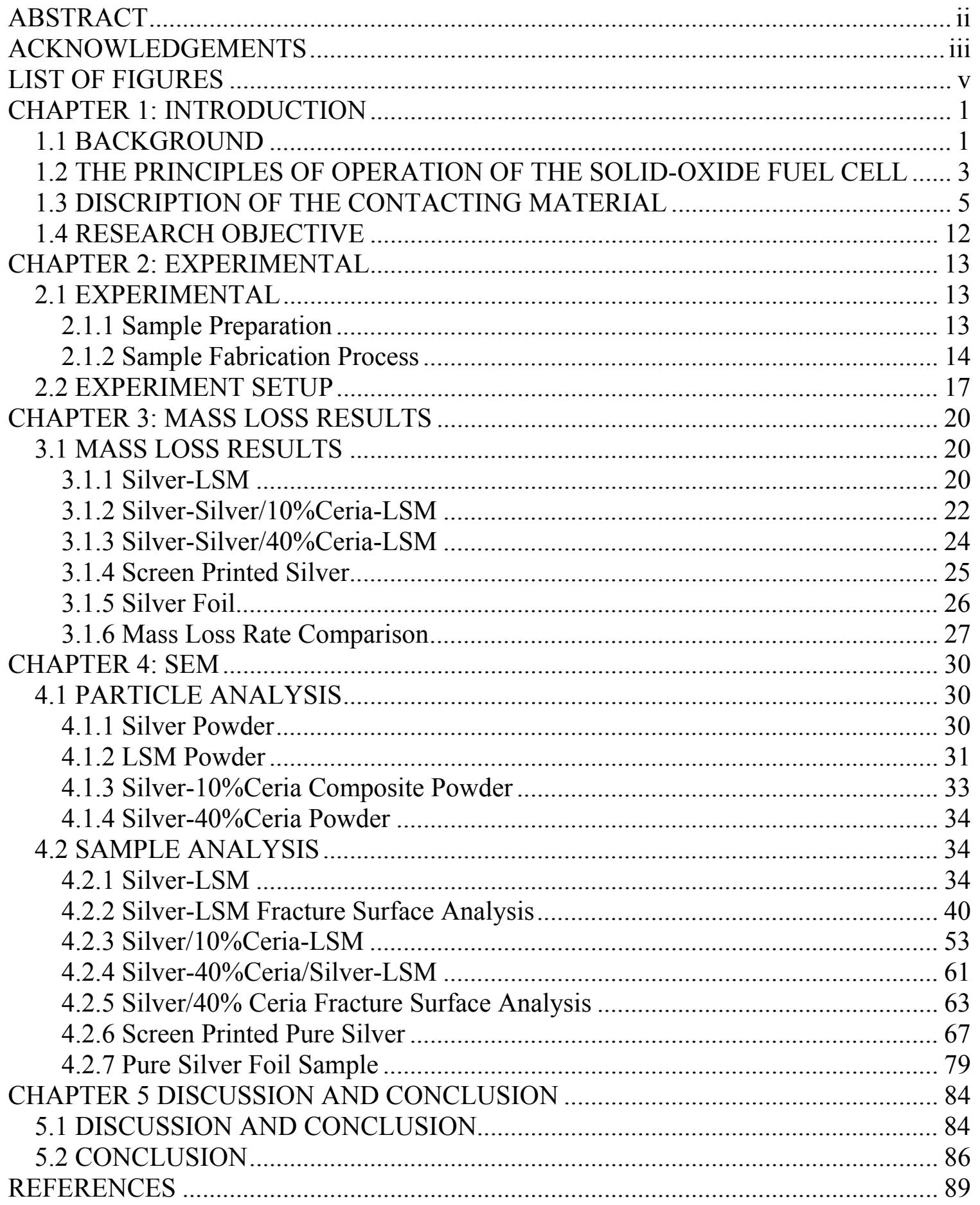




\section{LIST OF FIGURES}

Figure 1 Illustration of Chemical Reaction in a Solid-Oxide Fuel Cell ............................. 4

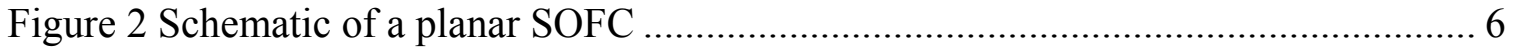

Figure 3 Contact area between the LSM cathode and interconnect.................................... 6

Figure 4 Manufacturing process for silver-LSM samples .............................................. 14

Figure 5 Manufacturing process for silver/ceria-LSM samples ...................................... 16

Figure 6 Binder burnout process.......................................................................... 17

Figure 7 Experiment apparatus and condenser system .................................................. 19

Figure 8 Mass loss of silver-LSM sample for first eleven weeks..................................... 20

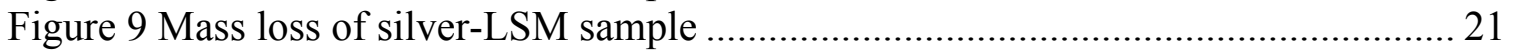

Figure 10 Mass loss of silver $/ 10 \%$ ceria-LSM sample .................................................... 23

Figure 11 Mass loss of silver/40\%ceria-LSM sample ................................................... 24

Figure 12 Mass loss of screen printed silver sample .................................................... 25

Figure 13 Mass loss of silver foil (50 microns) ....................................................... 27

Figure 14 Cumulative mass loss of all samples ............................................................ 28

Figure 15 Surface of silver powder showing small silver particles .................................. 31

Figure 16 Surface of LSM powder showing smaller LSM particles ................................ 32

Figure 17 Angular nature of LSM particles ..................................................................... 32

Figure 18 Surface of spherical silver particles showing ceria adhesion .......................... 33

Figure 19 Silver-40Ceria particles showing ceria adhesion ............................................. 34

Figure 20 SEM micrograph and backscatter of surface of pre-exposure silver-LSM

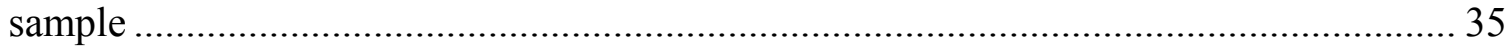

Figure 21 X-ray spectrum of pre-exposure silver-LSM surface ..................................... 36

Figure 22 SEM micrograph and backscatter of silver-LSM surface (1-week exposure) .37

Figure 23 SEM micrograph and backscatter of silver-LSM surface (2-weeks exposure) 37

Figure 24 SEM micrograph and backscatter of silver-LSM surface (3-week exposure) . 38

Figure $25 \mathrm{X}$-ray spectrum of silver-LSM surface after 3 weeks exposure showing

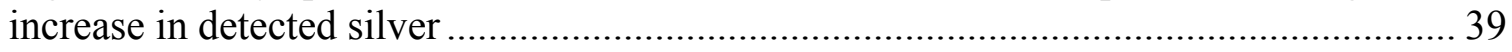

Figure 26 SEM micrograph of silver-LSM surface (12-weeks exposure)........................ 39

Figure 27 SEM micrograph of pre-exposed silver-LSM cross-section ............................. 41

Figure $28 \mathrm{X}$-ray map of the pre-exposure silver-LSM surface showing, left to right, silver

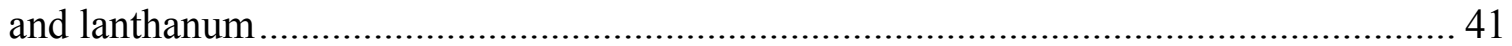

Figure 29 X-ray mapping overlay on the pre-exposure silver-LSM cross section........... 42 Figure 30 SEM micrograph and x-ray spectrum of pre-exposure silver-LSM cross section

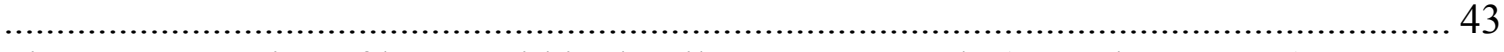

Figure 31 Location of layers within the silver-LSM sample (2-week exposure) ............. 44 Figure 32 SEM micrograph of silver-LSM cross section with spot analysis of silver layer

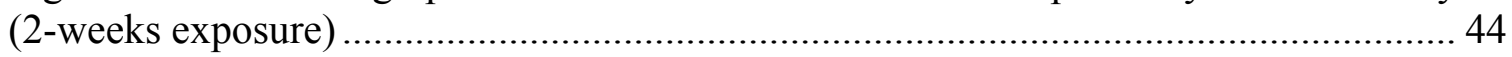

Figure 33 Comparison of $\mathrm{x}$-ray spectrum of entire surface vs. that of silver region (2-

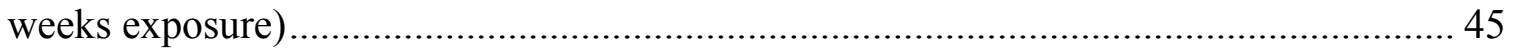
Figure 34 SEM micrograph and x-ray map of silver in silver-LSM cross-section (2-weeks

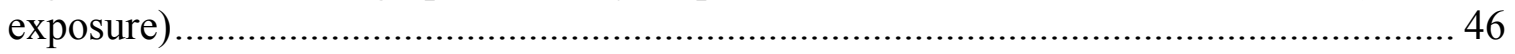
Figure 35 X-ray map of silver overlay of SEM micrograph of silver-LSM cross-section (2-weeks exposure) ……....................................................................................... 46 Figure 36 SEM micrograph of silver-LSM cross section at 1k and 3k magnification (12weeks exposure) 
Figure 37 SEM micrograph of silver-LSM cross section (14 weeks exposure) .............. 48

Figure $38 \mathrm{X}$-ray mapping of silver-LSM cross section documenting silver diffusion after

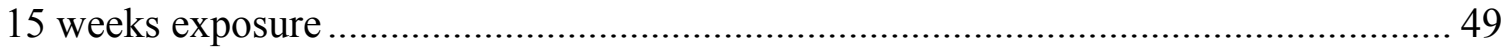

Figure 39 SEM micrograph at the center of the cross section of the silver-LSM sample

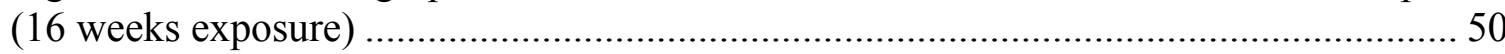

Figure 40 SEM micrograph of the LSM cross section of the silver-LSM sample (16

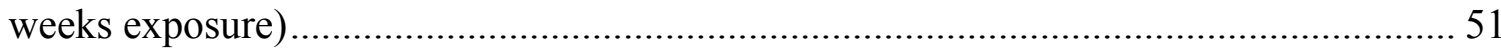

Figure 41 X-ray map for silver of LSM surface of the silver-LSM cross section (16 weeks

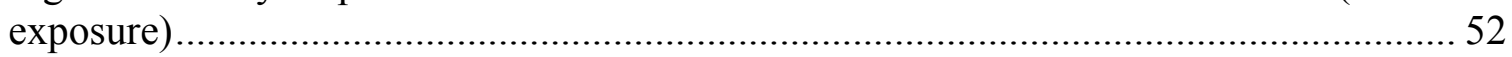

Figure 42 SEM micrograph with backscatter of pre exposure silver/10\%ceria with LSM

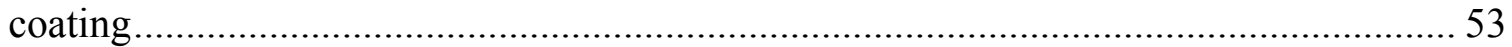

Figure 43 Crack analysis SEM micrograph with backscatter of silver/10\%ceria-LSM

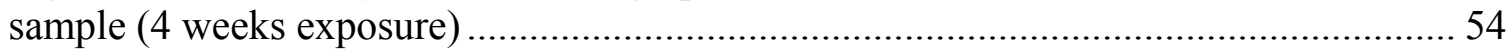

Figure 44 Crack analysis SEM micrograph with backscatter of silver/10\%ceria-LSM

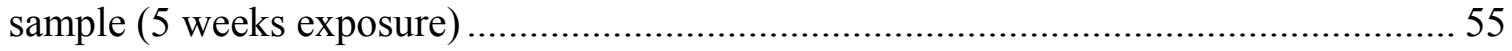

Figure 45 Crack analysis SEM micrograph of silver/10\%ceria-LSM sample (7 weeks

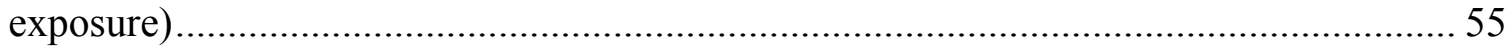

Figure 46 Crack analysis SEM micrograph of silver/10\%ceria-LSM sample (14 weeks

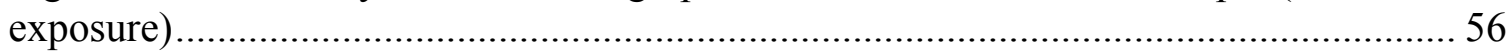

Figure 47 SEM micrograph and backscatter of silver/10\%ceria-LSM sample (5 week

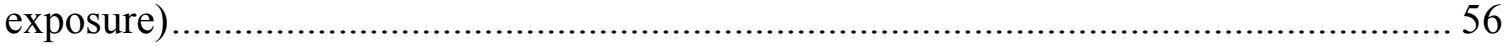

Figure 48 SEM micrograph and backscatter of silver/10\%ceria-LSM sample (7 week

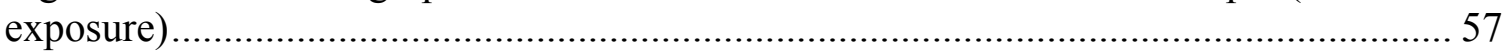

Figure 49 SEM micrograph and backscatter of silver/10\%ceria-LSM sample (10 week

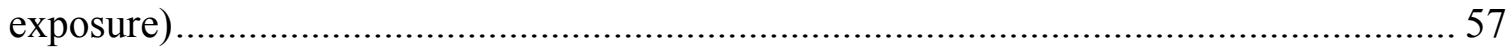

Figure 50 X-ray spectrum of entire silver/10\%ceria-LSM surface .............................. 58

Figure 51 SEM micrograph of silver/10\%ceria-LSM surface showing cracking............. 58

Figure 52 SEM micrograph and backscatter of silver/10\%ceria-LSM sample surface (4

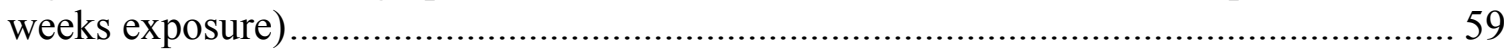

Figure 53 SEM micrograph and backscatter of silver/10\%ceria-LSM sample surface (5

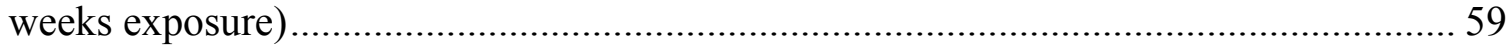

Figure 54 SEM micrograph and backscatter of silver/10\%ceria-LSM sample surface (7

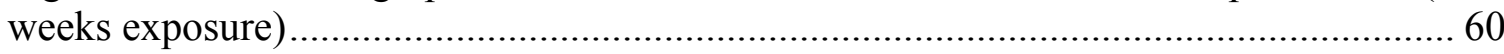

Figure 55 SEM micrograph and backscatter of silver/10\%ceria-LSM sample surface (14

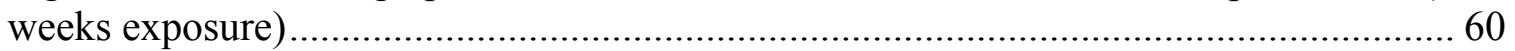

Figure 56 SEM micrograph and backscatter of pre exposure silver/40\%ceria-LSM sample surface

Figure 57 SEM micrograph and backscatter of silver/40\%ceria-LSM sample surface (3

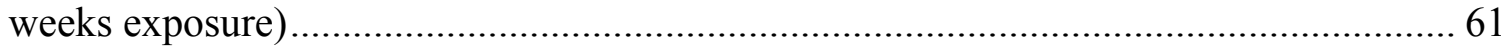

Figure 58 SEM micrograph and backscatter of silver/40\%ceria-LSM sample surface (4

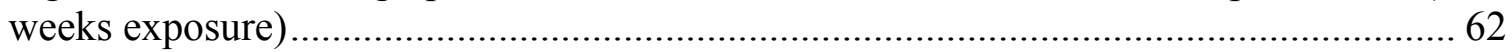

Figure 59 X-ray spectrum of silver/40\%ceria-LSM surface after 14 weeks exposure..... 62 Figure 60 Cross section SEM micrograph of the silver/40\%ceria-LSM sample (16 weeks

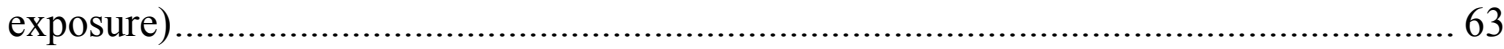

Figure $61 \mathrm{X}$-ray map of the cross section of the silver/40\%ceria-LSM sample (16 weeks exposure) 
Figure 62 SEM micrograph of the bottom of the LSM layer (16 weeks exposure) 65 Figure 63 X-ray map of the bottom of the LSM layer of the silver/40\%ceria-LSM sample (16 weeks exposure) 66

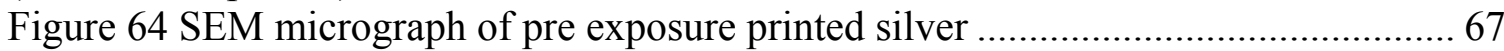

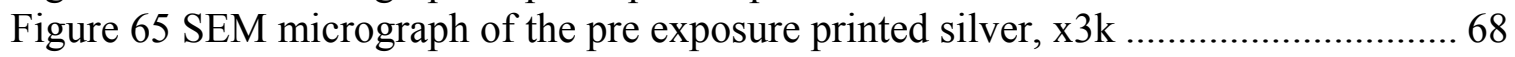

Figure 66 SEM micrograph of the printed silver after 1 day exposure ........................... 69

Figure 67 SEM micrograph of the printed silver after 1 day exposure showing faceting 69 Figure 68 SEM micrograph of the printed silver after 3 day exposure showing continuing

faceting..... 70

Figure 69 Comparison of faceting of silver between samples of 1 day and 3 day exposure

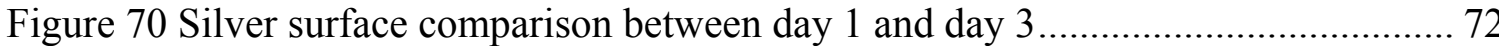

Figure 71 SEM micrograph of the silver surface (1 week exposure) ............................ 73

Figure 72 Printed silver after 1 week exposure showing more extensive faceting........... 74

Figure 73 SEM micrograph of printed silver surface showing extensive particle loss ( 8

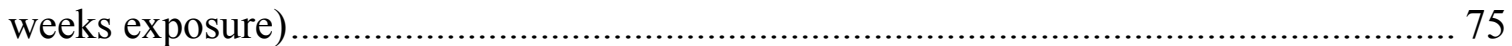

Figure 74 SEM micrograph of printed silver surface showing more extensive faceting (8

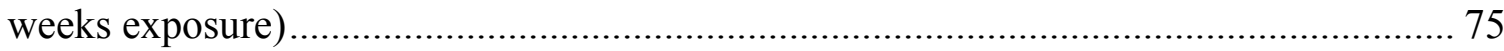

Figure 75 Description of evaporation of printed silver.............................................. 76

Figure 76 SEM micrograph of printed silver (16 week exposure) ................................ 77

Figure 77 SEM micrograph of printed silver showing more extensive faceting (16 week

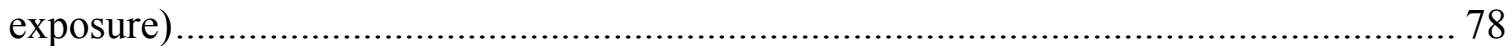

Figure 78 Evidence of small facets on surface of silver particles (16 week exposure).... 78

Figure 79 SEM micrograph of pre exposure silver foil surface.................................. 79

Figure 80 X-ray spot analysis of pre exposure silver foil surface ................................ 80

Figure 81 SEM micrograph of silver foil surface after 1 week exposure at $1 \mathrm{k}$ and $4 \mathrm{k}$

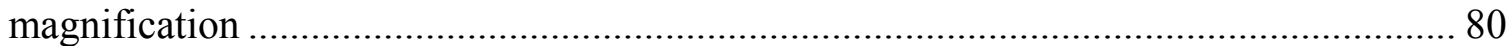

Figure 82 Pure silver surfaces after high temperature exposure................................. 82 


\section{CHAPTER 1: INTRODUCTION}

\subsection{BACKGROUND}

The solid-oxide fuel cell has great potential for service as a method of power generation. Able to operate at temperatures ranging from between $800{ }^{\circ} \mathrm{C}$ and $1000{ }^{\circ} \mathrm{C}$, the SOFC has advantages over other fuel cell types in the fact that it is able to operate on gasified hydrocarbon fuels or natural gas without preconditioning of the fuel, and represents the most efficient electric power generation method able to use multiple fuels ${ }^{1}$. The operating temperature allows for high reaction rates at relatively low cost and for the excess heat generated to be used for electrical power cogeneration. Furthermore, the use of a dense oxygen-ion conduction compound as the electrolyte simplifies the design of the SOFC over some other fuel cell types ${ }^{2}$.

There are two main configurations of SOFC stacking: planar and tubular. For the purposes of this study, the planar configuration was investigated. In planar fuel cell systems the current path of the electrons is drastically shorter, reducing total ohmic losses below that of the tubular arrangement and increasing overall power density. The cell itself is based upon stacks of thin flat plats, usually made of either ceramic or a metal or steel alloy. One particular advantage of the planar fuel cell is its low cost and ease of manufacturing, with the electrolyte and interconnect fabricated by tape casting, chemical vapor deposition or powder sintering. The electrodes are applied using slurry methods, screen printing or plasma spraying ${ }^{4}$.

There are four main components of a SOFC; anode, cathode, electrolyte and interconnect. In a planar SOFC, these components are stacked in flat layers in the 
following recurring order; interconnect, cathode, electrolyte, anode, and then the pattern begins again with the interconnect.

The state of the art material for the anode is a composite of $\mathrm{Ni}$ and yttriastabilized zirconia (YSZ), with the zirconia typically doped with 8 to $10 \mathrm{~mol} \%$ yttria. Ni is usually incorporated into the YSZ as a cerment ${ }^{2}$. The inclusion of Ni in the anode material serves to increase the electrochemical activity for $\mathrm{H}_{2}$ oxidation. YSZ reduces sintering of the Ni particles, thereby increasing the number of reaction sites by providing a three dimensional porous electrochemical reaction zone for the anode ${ }^{4,5}$.

The interconnect separates the gases used on the anode and cathode sides of the fuel cell. Formed of either ceramic or metal, the interconnect is ribbed to create gas channels in which the reactant gases travel. These ribs form a bipolar gas separator and also attach the anode to the cathode of adjoining cells ${ }^{4}$. As it is physically adhered to both the anode and the cathode, it is important that the interconnect be designed with a thermal expansion coefficient as close as possible to that of the other cell stack materials. For metal interconnect designs, it is important that oxidation resistance and chromium leaching be avoided ${ }^{3}$.

The cathode is designed to enhance the electrochemical breakdown of oxygen. Usually made of electrically conductive oxides or ion-conducting ceramics, the cathode must be non-reactive with the fuel and be able to operate at the low temperatures currently being achieved in SOFC's ${ }^{6}$. The cathode must also be highly conductive and both dimensionally and chemically stable at the high temperatures encountered during SOFC operation. Furthermore, the cathode must be compatible both reactively and in terms of thermal expansion coefficient with the materials it comes into contact with and 
must be sufficiently porous to serve as an oxygen-ion transport medium ${ }^{4}$. The typical material currently being investigated is strontium-doped lanthanum manganite (LSM) ${ }^{2}$.

The electrolyte is the core of the SOFC stack. Made of an oxygen-ion conducting material, the electrolyte allows the transfer of oxygen ions from the air on the cathode side to the anode side, generating an electrical voltage ${ }^{4}$. The electrolyte of the SOFC is most commonly made of YSZ, with other materials such as ceria ceramics being investigated as well ${ }^{13}$.

\subsection{THE PRINCIPLES OF OPERATION OF THE SOLID-OXIDE FUEL CELL}

The power generation of the SOFC occurs through electrochemical reactions utilizing $\mathrm{H}_{2}$ and $\mathrm{O}_{2}$. These reactions are described through the following equations ${ }^{14}$.

$$
\begin{aligned}
& \mathrm{H}_{2}+1 / 2 \mathrm{O}_{2} \rightarrow \mathrm{H}_{2} \mathrm{O}+2 e^{-}, \text {at the anode } \\
& 1 / 2 \mathrm{O}_{2}+2 e^{-} \rightarrow \mathrm{O}^{2-}, \text { at the cathode } \\
& \mathrm{H}_{2}+1 / 2 \mathrm{O}_{2} \rightarrow \mathrm{H}_{2} \mathrm{O} \text {, the overall cell reaction }
\end{aligned}
$$

Equation 1 Electrochemical Reactions

During normal operation, the oxygen supplied to the cathode side reacts with incoming electrons from the external circuit, forming oxygen ions. This reaction is surface area dependent, so a porous cathode is used to increase the reaction area. Modern electrolytes are constructed of microstructures of such high porosity that the effective surface area can be as high as thousands of times greater than their length $\mathrm{x}$ width ${ }^{2}$. The oxygen ions migrate through the electrolyte reacting with $\mathrm{H}^{+}$at the anode/electrolyte to form $\mathrm{H}_{2} \mathrm{O}$, 
releasing two electrons for each $\mathrm{H}_{2} \mathrm{O}$ molecule formed. These liberated electrons then flow around the external circuit attached to the cell, forming an electrical current which eventually returns to the cathode. At this point, the electrons react with a single $\mathrm{O}_{2}$ molecule to form two oxygen ions. It is important that the centralized electrolyte of the SOFC be both impervious to electrons and $\mathrm{H}^{+}$ions, allowing only $\mathrm{O}^{2-}$ to pass through it. This ensures that electrons are forced to pass around the entire circuit and not simply return through the electrolyte, which would result in no electric power generation, as well as preventing the reaction of the $\mathrm{H}^{+}$and $\mathrm{O}^{2-}$ ions from occurring before the anode. This process is presented in Figure $1{ }^{14}$.

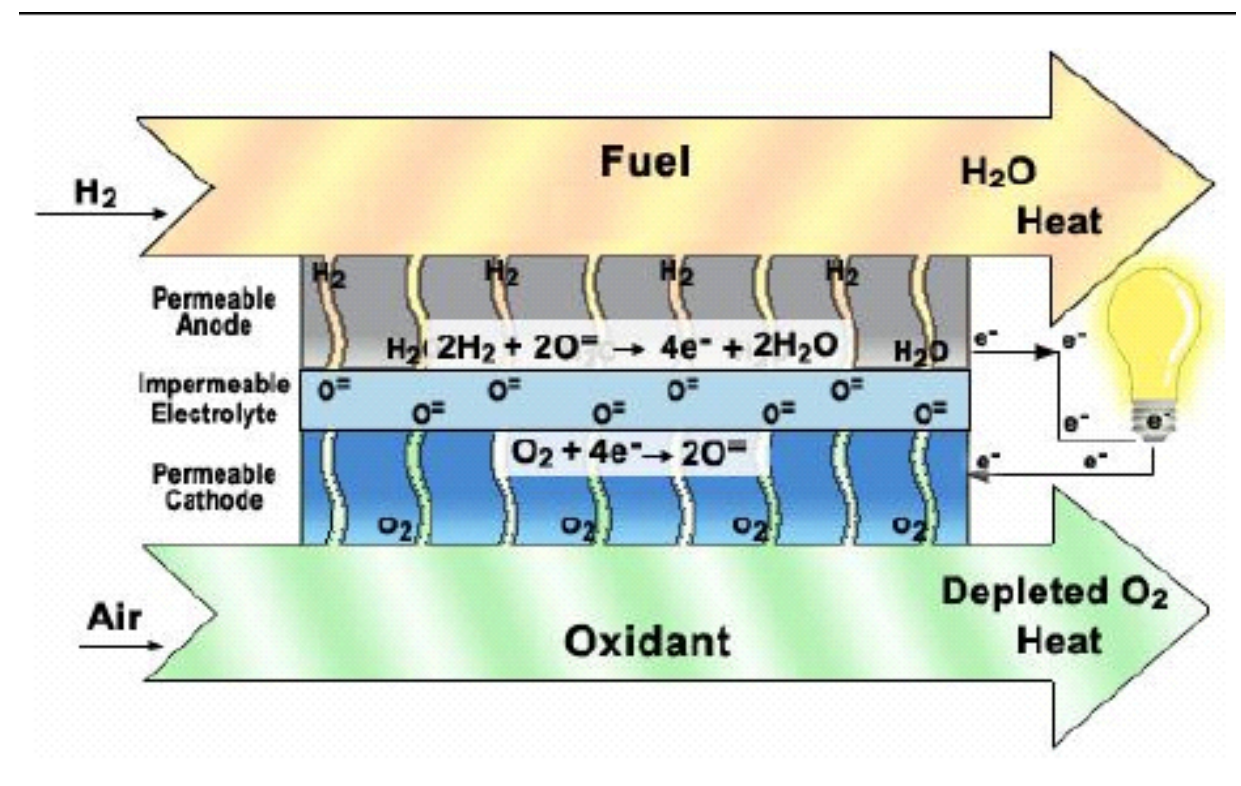

Figure 1 Illustration of Chemical Reaction in a Solid-Oxide Fuel Cell

The transfer of oxygen ions through the electrolyte is responsible for electric power generation of the SOFC. The operational voltage of the SOFC is total open circuit voltage minus all losses that occur through the circuit, with the main losses being ohmic, activation, mass transport and fuel cross over ${ }^{2}$. Ohmic losses are caused by resistance to 
the electron flow through the cell components. Activation losses are the voltage required to fuel the chemical reaction and the electrode surfaces, while crossover losses are the energy potential lost by fuel passing through the electrolyte. As the two reactants travel through the cell, the concentration of the reactants decreases as fuel is used. This reduction on reactant concentration results in mass transport losses ${ }^{2}$. The cell operating voltage is calculated using the following equation;

$$
V_{\text {operating }}=V_{\text {OCV }}-\Delta V_{\text {ohm }}-\Delta V_{\text {act }}-\Delta V_{\text {trans }}-\Delta V_{f c}
$$

Equation 2 Calculation of the cell operating voltage

The Ohmic losses of the cell are dependent upon the materials used in the fabrication of the cell stack, the geometry of the cell components ${ }^{14}$, as well as the length of the current collection path ${ }^{7}$. As any increase in the resistance of the materials will lead to a decreased operating voltage, it is very important that low resistance materials be used to manufacture the cell components and by minimizing the thickness of the cell components.

\subsection{DISCRIPTION OF THE CONTACTING MATERIAL}

In order to maintain effective electrical connections between the cathode and the interconnect during cell operation, contact materials are often used to ensure adhesion and connectivity. This is important as the interconnect often serves as a current collector for the entire cell stack ${ }^{7}$. The region of the contact material is illustrated as the circled area in the following diagram ${ }^{14}$. 


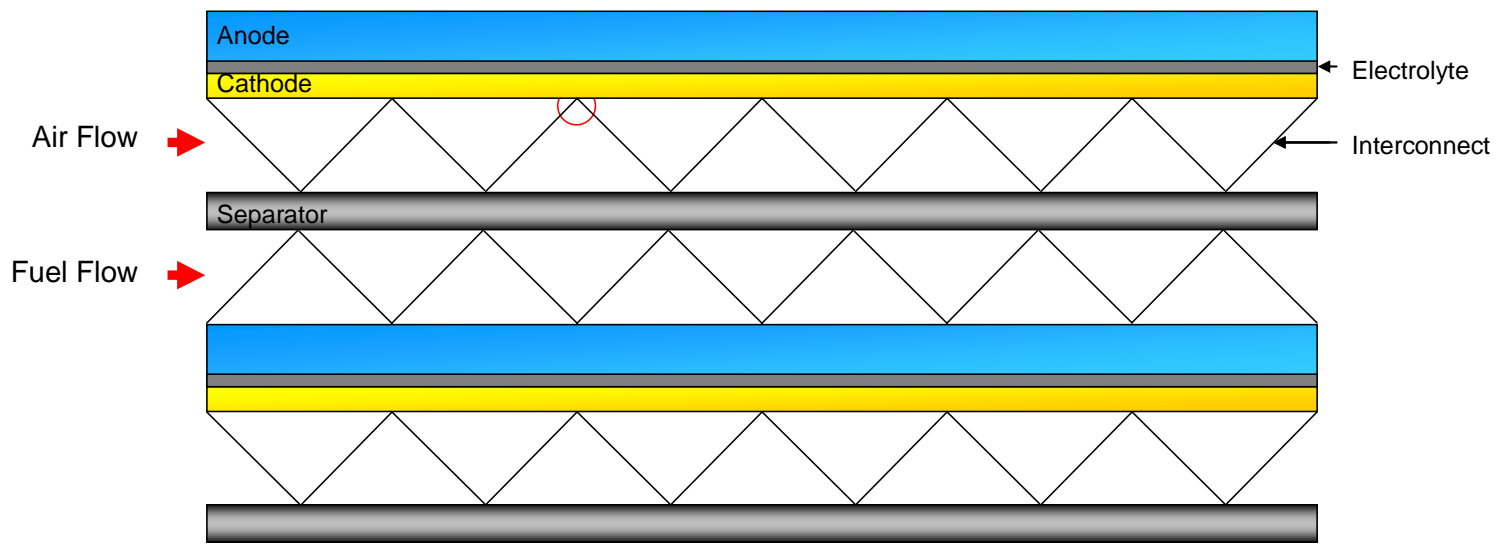

Figure 2 Schematic of a planar SOFC

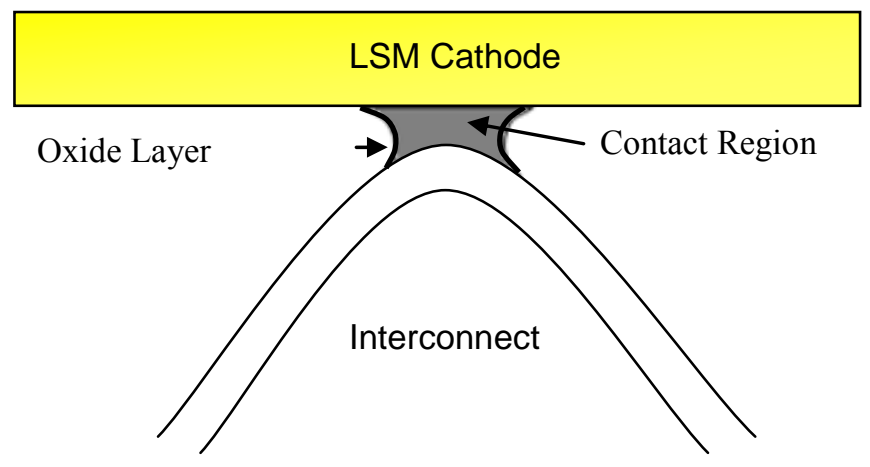

Figure 3 Contact area between the LSM cathode and interconnect

More specifically, the contact region of the cathode chamber is shown in the figure above. As it serves as a current collection point, oxidation of the material used as the contact paste is a concern if non-conductive oxide scales form, breaking the electrical path between the cathode and interconnect. The high operating temperature of the SOFC makes the cathode an oxidizing environment. This rules out many metals as potential contact paste materials as metals tend to form oxide scales. Also, the high temperature of the cathode region makes evaporation of the contact material a concern, especially if metals are to be used. 
Materials currently considered for use as a contact material are platinum, LSM, LSC (strontium-doped lanthanum cobalite) and LSF (strontium-doped lanthanum ferrite). However, there are other materials that are becoming candidates for use due to their considerably lower cost than the above materials.

In recent years, silver has become a viable contact material option due to the lowering of SOFC operating temperatures to below $800{ }^{\circ} \mathrm{C}$. Silver is of particular interest because it is highly ductile, highly conductive and is a low cost material relative to other SOFC materials. Furthermore, silver does not form oxide scales in an oxidizing environment, such as that present in the cathode chamber of a SOFC. Of concern is the fact that the current operating temperature of the SOFC is near the $961.78{ }^{\circ} \mathrm{C}$ melting temperature of silver, which brings the volatility of silver into question. Also, silver has been determined to have a degree of diffusivity at high temperature, making it important to both determine the rates of silver diffusivity and its effect upon cell operating parameters before it can effectively be used in a $\mathrm{SOFC}^{15,16}$. Silver migration has also been found to be electrochemically activated, a problematic phenomenon for any material being considered for use as a current collector ${ }^{16}$.

Silver is generally considered to be too soft for most uses in a pure form. As a result, most silver in use is sterling silver, which is most often a silver-copper alloy, but is sometimes a silver-zinc alloy as well. The composition of sterling silver is usually between $13-5 \mathrm{wt} \%$ copper-silver, with $7.5 \mathrm{wt} \%$ copper-silver generally the most frequently encountered alloy. Copper increases the strength and durability of the silver, facilitating the production of complex items that will be able to retain their shape with use, as well as greatly reducing the material cost. 
Strontium $10 \%$-doped lanthanum manganite (LSM) is currently one of the most widely used materials for cathode applications in a SOFC. Upon high temperature firing, LSM produces perovskite structures and will partially sinter to form a porous cathode ideal for increased cathode side reactions in fuel cell due to increased reaction area. It is both highly stable and electrically conductive.

Ceria, or cerium(IV) oxide, $\mathrm{CeO}_{2}$, is widely used to improve catalytic reactions at low temperature ${ }^{10}$. Major applications of ceria include use as a three way catalyst in automobile exhaust systems and the removal of organics from waste water ${ }^{11}$. An excellent oxygen transport vehicle, ceria has high electronic conductivity. Ceria is highly stable at SOFC operating temperatures, melting at approximately $2100^{\circ} \mathrm{C}$. The doping of ceria with other transition metals, such as silver, increases the oxygen transport capacity of the material ${ }^{10}$.

Contacting material research comparing the use of platinum, LSM, LSC and LSF with respect to area specific resistance (ASR) was conducted at the Pacific Northwest National Laboratory (PNNL) ${ }^{9}$. In the PNNL study, testing was conducted at $800{ }^{\circ} \mathrm{C}$ and with the materials applied between Crofer22 APU coupons and LSF cathodes. Operating without a contacting material provided the worst performance. Platinum was determined to have the best performance, but is cost prohibitive, while LSM was found to have the lowest rate of increase in ASR. In order for the SOFC to be established as an option for commercial power generation, it is important that lower cost contact material options be established. Silver was not examined in this study ${ }^{9}$.

In Liu et al, a $12 \mu \mathrm{m}$ DC sputter coated silver layer was studied during 8 hours of exposure to SOFC operating condition. It was determined that oxygen permeation 
through dense silver membranes increases with temperature. This permeation has also been shown to follow an Arrhenius relation with an activation energy of $0.94 \mathrm{eV}(21.7$ $\left.\mathrm{kcal} \mathrm{mol}^{-1}\right)^{23}$. Degradation of the silver surface occurred at the grain boundaries of the silver sample.

Silver evaporation studies of silver meshes exposed to air flow at $800{ }^{\circ} \mathrm{C}$, as well as to inert gases, for 500 hours indicated that silver evaporation is much greater in air than in inert gas. Also, due to silver not forming oxide scales at high temperature during long-term exposure, a low ASR was maintained ${ }^{7}$. No oxides or evaporative barriers were used to reduce the mass loss rate of silver in this study.

Research into the use of other metal oxides to inhibit silver evaporation was conducted using $92.5 \mathrm{wt} \% \mathrm{Ag}, 7.5 \mathrm{wt} \% \mathrm{Cu}$ sterling silver as a contact material. Samples of sterling silver were exposed at $3 \mathrm{l} / \mathrm{min}$ air flow at $800{ }^{\circ} \mathrm{C}$. Data indicated that when exposed to an oxidizing environment, the copper formed copper-oxide $\left(\mathrm{CuO}_{2}\right)$ particles which coarsened throughout the bulk of the sample through the duration of the long term experiment. Reactivity of copper-oxides with the exposed silver increased silver evaporation caused the sterling silver to evaporate at a rate twice that of pure silver ${ }^{14}$.

Time series analysis of LSM pressed into silver using SEM showed that LSM did not coarsen in high temperature conditions. This seems to indicate that LSM may serve as a superior evaporation inhibitor for SOFC applications ${ }^{14}$.

Previous research conducted by $\mathrm{McCool}$ and Lin determined that a sputter deposited bimetallic nanostructured membrane of palladium and silver does not have high gas permeability. However, these membranes have an increase in gas permeability if annealed at high temperature. The corresponding increase in grain size that occurs during 
annealing was determined to narrow the grain boundary gap of the Pd-A, which improved the perm-selectivity for gas diffusion ${ }^{20}$.

The oxygen permeation flux of a perovskite type ceramic membrane, such as an LSM cathode, was determined to increase considerably with decreasing grain $\operatorname{size}^{17}$. In this study, the effect of membrane microstructure on the oxygen permeability of $\mathrm{SrCo}_{0.8} \mathrm{Fe}_{0.2} \mathrm{O}_{3-\delta}$ (SCFO) was investigated with sintering temperatures of 950 to $1200{ }^{\circ} \mathrm{C}$. It was determined that for materials sintered at $950{ }^{\circ} \mathrm{C}$, the majority of the SCFO grains were around 2-4 $\mu \mathrm{m}$ in diameter, while it the grains were 8-10 $\mu \mathrm{m}$ in diameter for those sintered at $1200{ }^{\circ} \mathrm{C}$. The oxygen permeability of the SCFO was found to be highest for the $950{ }^{\circ} \mathrm{C}$ sintered membrane. Through microstructure analysis of the SCFO samples it was determined that the densities of the samples were similar, so that the primary differences between the samples were in terms of microstructure. Zhang et al concluded that it was possible that the surface exchange rate of oxygen was enhanced by the increase in interfaces on the sample surface that would exist with smaller grain sizes. Also, the increase in grain boundaries creates a higher concentration of defects and defect jump rates that could provide a faster diffusion path ${ }^{17}$.

In Simner et al, atomized silver spheres were coated with a $1 \mu \mathrm{m}$ thick layer of $\left(\mathrm{La}_{0.6} \mathrm{Sr}_{0.4}\right)_{0.98} \mathrm{Fe}_{0.8} \mathrm{Co}_{0.2} \mathrm{O}_{3}$ (LSCF -6428) cathode powder in an attempt to reduce silver diffusivity and/or volatility ${ }^{15}$. Silver spheres were coated with LSCF using mechanofusion and deposited on anode supported YSZ electrolytes and exposed in a cathode environment at $700{ }^{\circ} \mathrm{C}$. Some diffusion of silver occurred, with silver collecting on the outer portion of the LSCF coating. The silver diffusion appeared to be both temperature and current dependent, with diffusion occurring slower as temperature and 
current decrease. However, the silver-LSCF interface remained intact throughout testing, with no increase in the porosity of the interface, regardless of diffusion rates. Power densities were initially lower than $100 \mathrm{mWcm}^{-2}$, but after substantial conditioning of the samples, the performance of the silver greatly increased $\left(>500 \mathrm{mWcm}^{-2}\right)$. It was suggested that the diffusion of silver to the surface of the coating layer increased the cathode-electrolyte contact area and was responsible for the increase in power density after conditioning. It was noticed that after conditioning, significant power degradation occurred at the rate of $0.016 \%$ per hour, an unacceptably high rate. It seems that, while some silver diffusion to the electrolyte is beneficial in extending the active electrolytecathode interface, further diffusion of silver could serve to reduce the triple phase boundary length, resulting in power degradation ${ }^{15}$.

Other research has determined that a LSCF ceramic coating of nanoparticle silver for use as the cathode material of the SOFC resulted in a reasonable power density between $750{ }^{\circ} \mathrm{C}$ and $650{ }^{\circ} \mathrm{C}^{16}$. The cathode initially had a low power density $(<100 \mathrm{~mW}$ $\mathrm{cm}^{-2}$ ), but after a conditioning period, power density increased to $500-650 \mathrm{~mW} \mathrm{~cm}{ }^{-2}$. The length of the conditioning period was determined to be temperature dependent. The conditioning period was found to be $20 \mathrm{~h}$ at $800^{\circ} \mathrm{C}$, and greater than $100 \mathrm{~h}$ at $700{ }^{\circ} \mathrm{C}$. After the completion of conditioning, a power degradation rate of $7 \%$ per $1000 \mathrm{~h}$ at $750{ }^{\circ} \mathrm{C}$ and $3-4 \%$ at $700{ }^{\circ} \mathrm{C}$ transpired. No degradation was found at $650{ }^{\circ} \mathrm{C}$. The instability of the power density was determined to be related to the excessive migration of silver at higher operating temperatures. This resulted in a decrease in the triple phase boundary and a restriction of air access to the cathode electrolyte interface ${ }^{16}$. 


\subsection{RESEARCH OBJECTIVE}

The objective of this research is the development and long term testing of cathode-interconnect contact materials. Particular attention will be paid to the utilization of silver and silver/ceramic composite test specimens. Due to the volatility of silver at the operating temperature of the SOFC, the mass loss of silver will be monitored, as well as the migration of silver into to the ceramic layers of the composite specimen. 


\section{CHAPTER 2: EXPERIMENTAL}

\subsection{EXPERIMENTAL}

\subsubsection{Sample Preparation}

The samples used in this experiment consisted of screen printed layers of silver, ceria and LSM in the form of a functionally graded sample, onto an YSZ substrate. The substrate began as a 1.1 " diameter disk, which was cut down to an oval of 0.75 " in width. The YSZ disks were then cleaned in a bath of aqua regia and rinsed in acetone to remove any contaminants.

A number of material powders were used in the preparation process. Cerium(IV) oxide powder (99.95\% pure) $\left(\mathrm{CeO}_{2}\right)$ nominal particle size of 50-100 nm; Strontium10\%doped lanthanum manganite, nominal particle size of $50 \mathrm{~nm}$; silver $(99.998 \%$ pure), nominal size $50 \mathrm{~nm}$. Mixtures of ceria and $\mathrm{Ag}$ in a variety of percentages were also used, with the percent ceria of the two mixtures used being silver/10\%ceria and silver $/ 40 \%$ ceria. These mixtures were made at the Pacific Northwest National Laboratory using a glycene nitrate process to ensure consistent mixing of the materials. The inks used in the fabrication of the samples for this experiment were made at the National Energy Technology Laboratory in Morgantown, WV. Alpha-terpineol was used as the binder. 


\subsubsection{Sample Fabrication Process}

A layer of silver was screen printed onto each YSZ disk that was made. This layer was $13 \mathrm{~mm}$ in diameter and resulted in a layer thickness of between 7 and $10 \mu \mathrm{m}$. From this point, three pairs of samples were made, with the composition of each pair differing from this step. To the first pair, a second layer of silver was deposited, bringing the total thickness of silver to approximately $15 \mu \mathrm{m}$. On top of the layer of silver, three $15 \mathrm{~mm}$ diameter layers of LSM were printed, with the layers centered on the silver layers so that no silver was exposed around the edges of the sample. The total thickness of the LSM layers was approximately $20 \mu \mathrm{m}$.

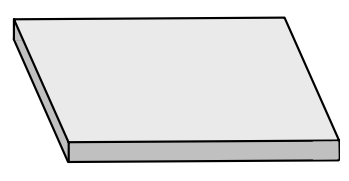
Step 1: YSZ
Substrate

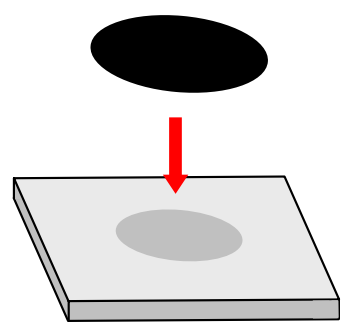

Step 3: Printing of LSM Layer

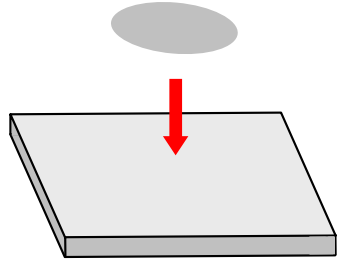

Step 2: Printing of Silver Cerment

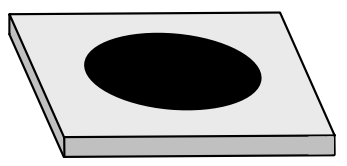

Step 4: Finished Sample, Binder Burn Off

Figure 4 Manufacturing process for silver-LSM samples

The second pair of samples had a $13 \mathrm{~mm}$ diameter layer of silver/10\%ceria ink deposited on the previously printed layer of silver. On top of this, three $15 \mathrm{~mm}$ diameter 
layers of LSM were printed on the sample in the same fashion as the first pair. The thickness of the LSM coating layer was roughly $20 \mu \mathrm{m}$

The third sample pair had a $13 \mathrm{~mm}$ diameter layer of silver $/ 40 \%$ ceria ink printed on top of the single silver layer. As in the second sample pair, three $15 \mathrm{~mm}$ diameter layers of LSM were printed on the sample. The thickness of the LSM coating layer was roughly $20 \mu \mathrm{m}$.

A description of the generic ceria sample manufacturing process is presented in the following figure. The same general process took place for each sample, with the only difference being the use of $10 \%$ or $40 \%$ ceria. 


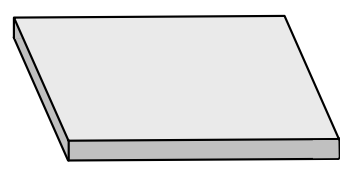

Step 1: YSZ

Substrate

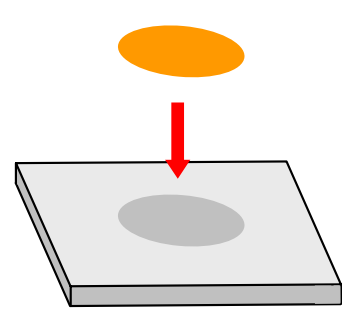

Step 3: Printing of $\mathrm{Ag}-\mathrm{CeO}_{2}$ Layer

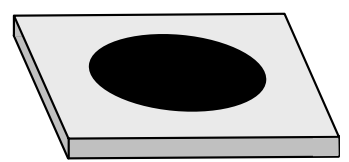

Step 5: Finished Sample. Binder Burn off.

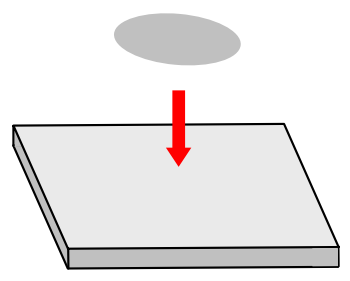

Step 2: Printing of Silver Cerment

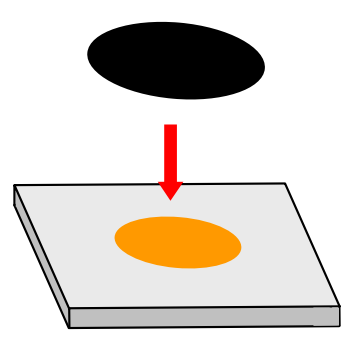

Step 4: Printing of LSM Layer

Figure 5 Manufacturing process for silver/ceria-LSM samples

Between the application of each screen printed layer, the sample was heated to approximately $200{ }^{\circ} \mathrm{C}$ using a heat gun at low setting to dry the binder for subsequent printing. After the samples were finished printing, they went through a two stage binder burnout process, the heating process of which is detailed Figure 6. 


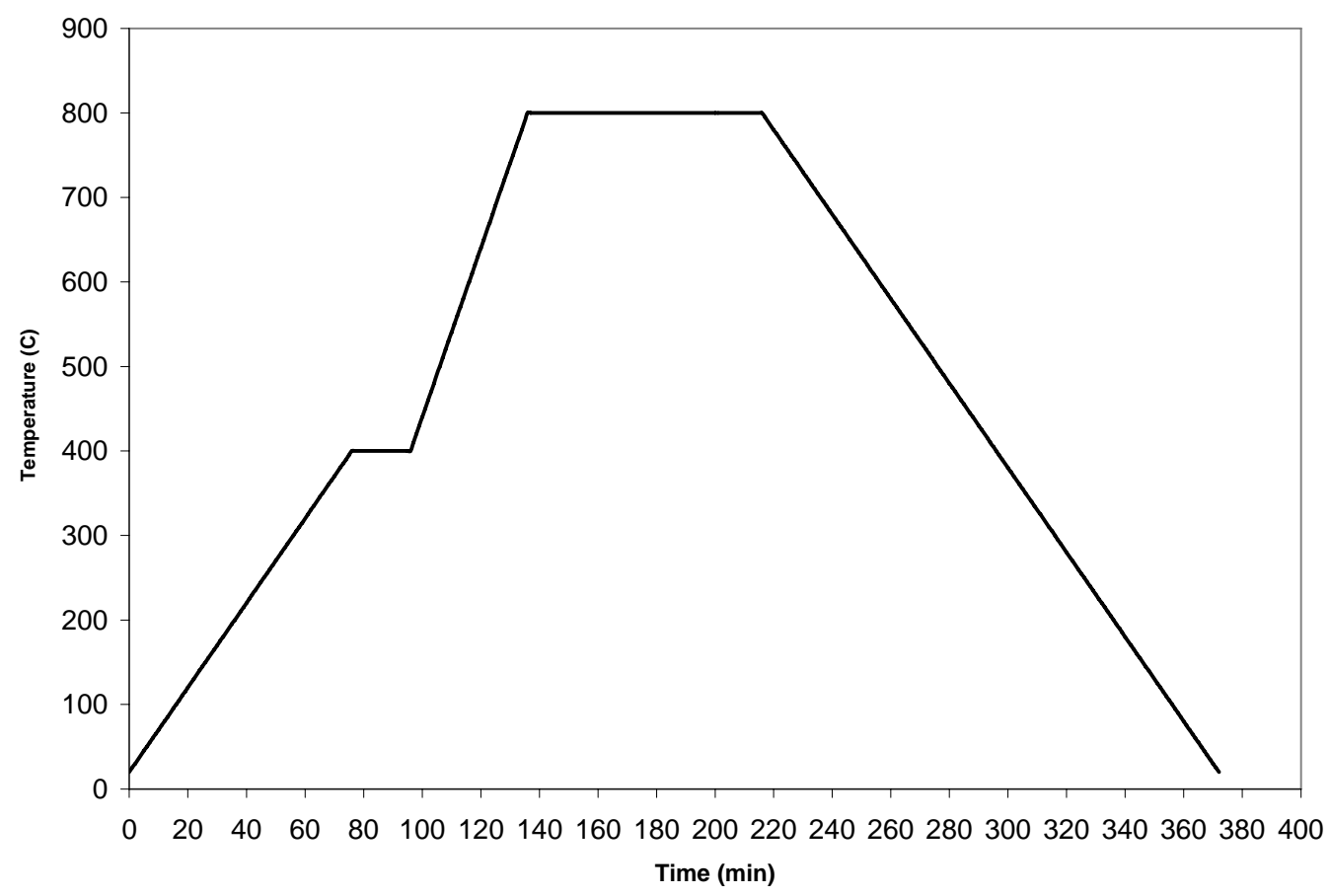

Figure 6 Binder burnout process

The burnout comprised of firing the samples to a temperature of $400{ }^{\circ} \mathrm{C}$ at a rate of $5{ }^{\circ} \mathrm{C} / \mathrm{min}$. A lag time of $20 \mathrm{~min}$ occurred at $400{ }^{\circ} \mathrm{C}$, with the temperature being increased at a rate of $10{ }^{\circ} \mathrm{C} / \mathrm{min}$ to a final burnout temperature of $800{ }^{\circ} \mathrm{C}$ to allow for enhanced organic decomposition.

\subsection{EXPERIMENT SETUP}

The apparatus used in the process of this research were tube furnaces used to simulate the cathode chamber environment of the SOFC. Air mass-flow rate and furnace temperature were maintained at $3 \mathrm{1} / \mathrm{min}$ and $800{ }^{\circ} \mathrm{C}$, respectively, throughout the long term exposure testing through the use of a Sierra Smart-Trak Series 100 flow meter and a temperature controller. Incoming air passes through a $5 \mu \mathrm{m}$ filter and oil and water 
desiccator to remove contaminants. The air supply was compressed atmospheric air. Two tube furnaces were used to expose the samples, with identical air flow and temperatures in each.

One of the primary data measurements in this experiment was the mass loss rate of Ag from the material of the sample. However, because the principal samples used in this study were composites of silver, $\mathrm{LSM}$, and $\mathrm{CeO}_{2}$, the mass loss each week would contain at least trace amounts of each material. In light of this, a mass collection system was designed to condense the evaporated material from the furnace exit gases. This system was applied to furnace one, which had a tube system arrangement passing through it divided into four separate channels. The four channels allowed for sample types to be isolated in the furnace and to have the evaporated material from individual samples collected. These tubes were $26 \mathrm{~mm}$ ID fused quartz tubes. On the end of each tube, a graded quartz to Pyrex seal was fused, to which a long U-shaped, Pyrex $26 \mathrm{~mm}$ ID condenser tube was attached. A filter of $3 \mathrm{~mm}$ quartz beads were placed in the bend of the condenser tube. This filter and condenser arrangement served to collect and condense the evaporated material from the samples. At the end of the experiment timeline, the evaporated material will be collected and analyzed to determine the mass of evaporated material and the composition of the condensate in order to determine the mass loss rates of each material used in the sample. 


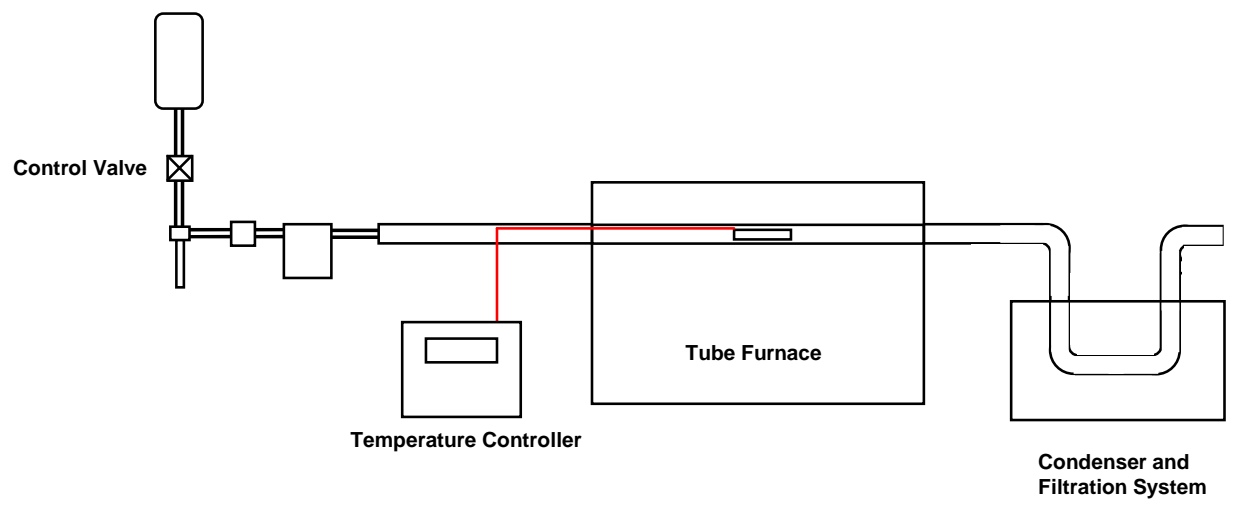

Figure 7 Experiment apparatus and condenser system

The second furnace that was used contained samples of singular material composition. All mass loss from these samples can safely be assumed to be known, making the addition of a mass collection system to the second tube furnace unnecessary. Exiting gas from the second furnace is expelled into the room.

Samples were placed in the furnace on machinable zirconia holders. Gaps of $1 / 4$ " were maintained between the samples to ensure that particles did not blow off of one sample and onto a down stream sample.

All samples were removed from the furnace weekly to monitor their condition, for SEM analysis, and to conduct mass loss measurements. All SEM analysis was conducted using coordinate measurements 
CHAPTER 3: MASS LOSS RESULTS

\subsection{MASS LOSS RESULTS}

\subsubsection{Silver-LSM}

After the silver-LSM sample experiences a mass increase due to oxidation of the LSM, the samples lose mass at a stable rate for the first eleven weeks. The mass loss of the silver-LSM specimen for the first eleven weeks is presented in the figure below.

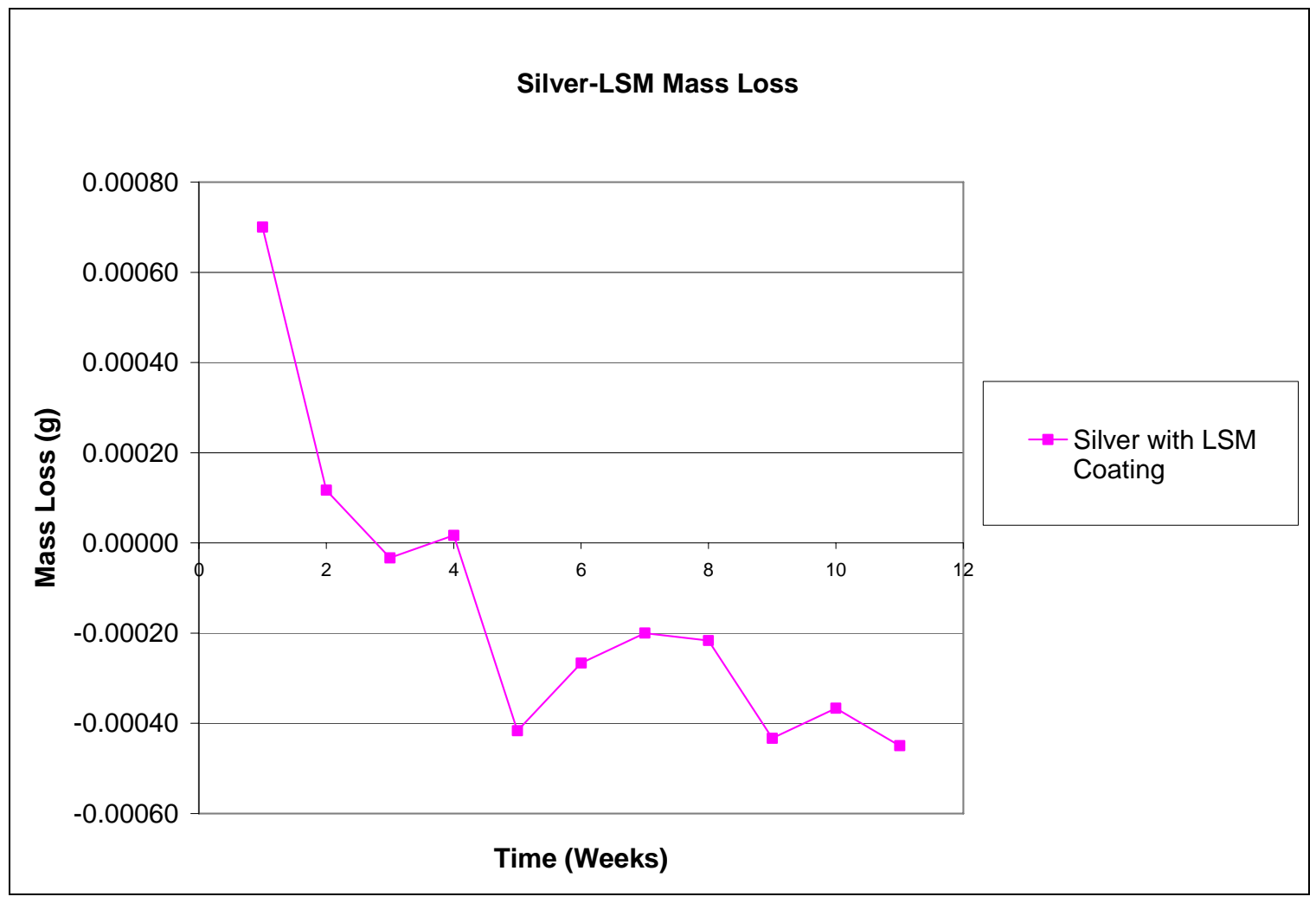

Figure 8 Mass loss of silver-LSM sample for first eleven weeks 
The mass loss rate of the silver-LSM sample is tolerable over the course of the first eleven weeks of exposure. The initial mass gain of the sample is likely caused by the oxidation of the LSM layer.

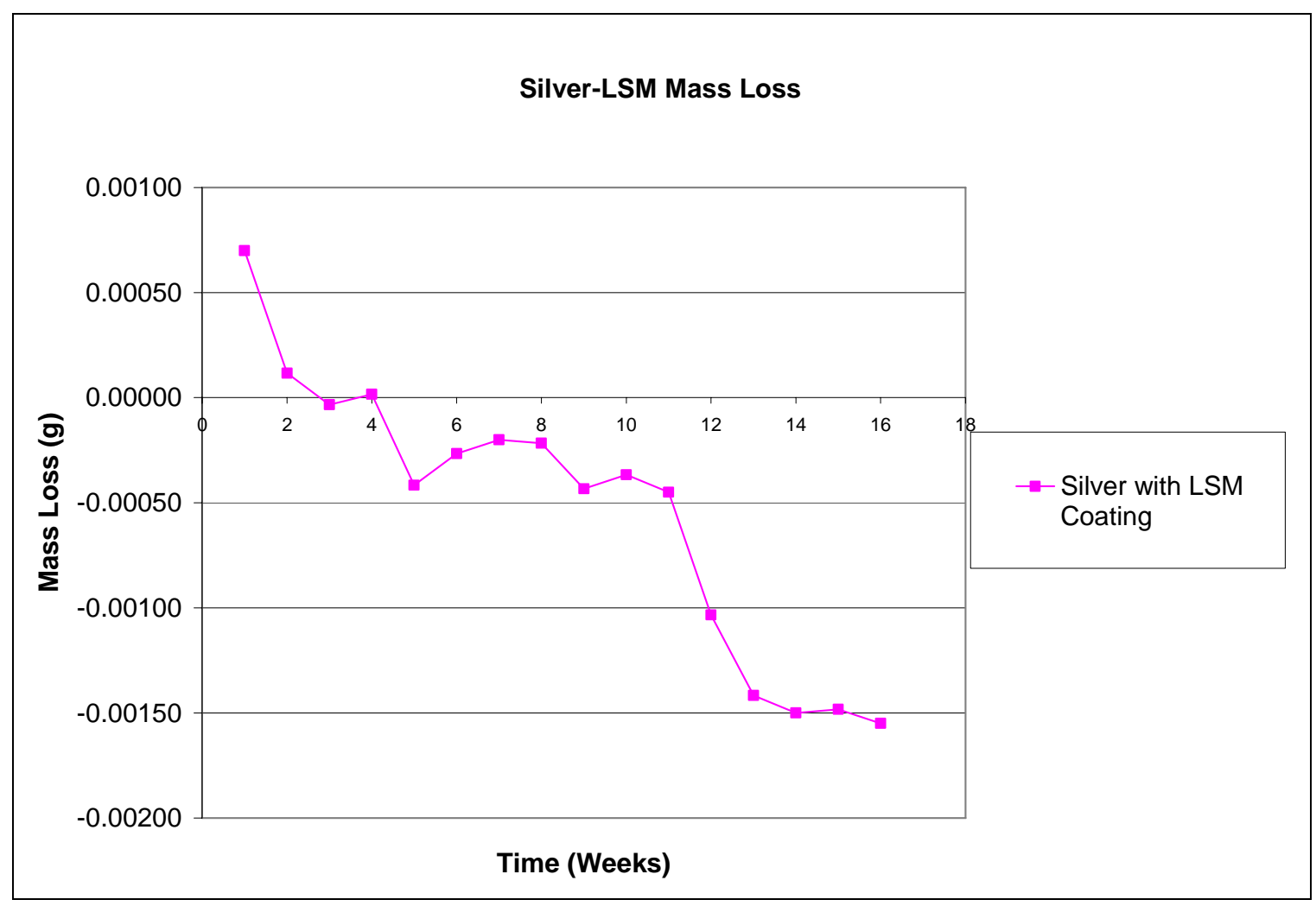

Figure 9 Mass loss of silver-LSM sample

From the plot it appears that the reduction of the sample is non-linear over the duration of the exposure time, with the mass loss rate displaying a seemingly increased mass loss after the eleventh week. A roughly linear trend of the first eight weeks of the exposure indicates that the mass loss rate is approximately $0.0040 \mu \mathrm{g}$ per week. Between week eleven and thirteen, the sample loses about $0.4833 \mu \mathrm{g}$ in mass each week, for an average over the entire exposure time of $0.0969 \mu \mathrm{g}$ per week. The apparent increase in the mass loss rate between week eleven and thirteen corresponds to a drastic $1200 \%$ increase. An approximation of the total silver mass loss at the higher rate was made using 
a linear trend line over the 40,000 hour (240 weeks) target life span of the material found that the sample would lose around $23.25 \mu \mathrm{g}$ in mass.

The large increase in the mass loss rate of the silver-LSM sample provides evidence that silver diffusion is occurring through the LSM layer, increasing the amounts of silver exposed to the air flow. This indicates that if silver diffusion is occurring, it will have taken place in significant amounts between weeks 3 and 11. The sudden increase in the mass loss rate of the sample indicates that silver does not lose mass in a uniform, linear manner, but rather has a non-linear mass loss rate.

\subsubsection{Silver-Silver/10\%Ceria-LSM}

The screen printed silver-silver-10\%ceria-LSM samples followed a trend of greater mass loss that of the silver-LSM samples, with a general mass loss trend rapidly established for the initial period. 


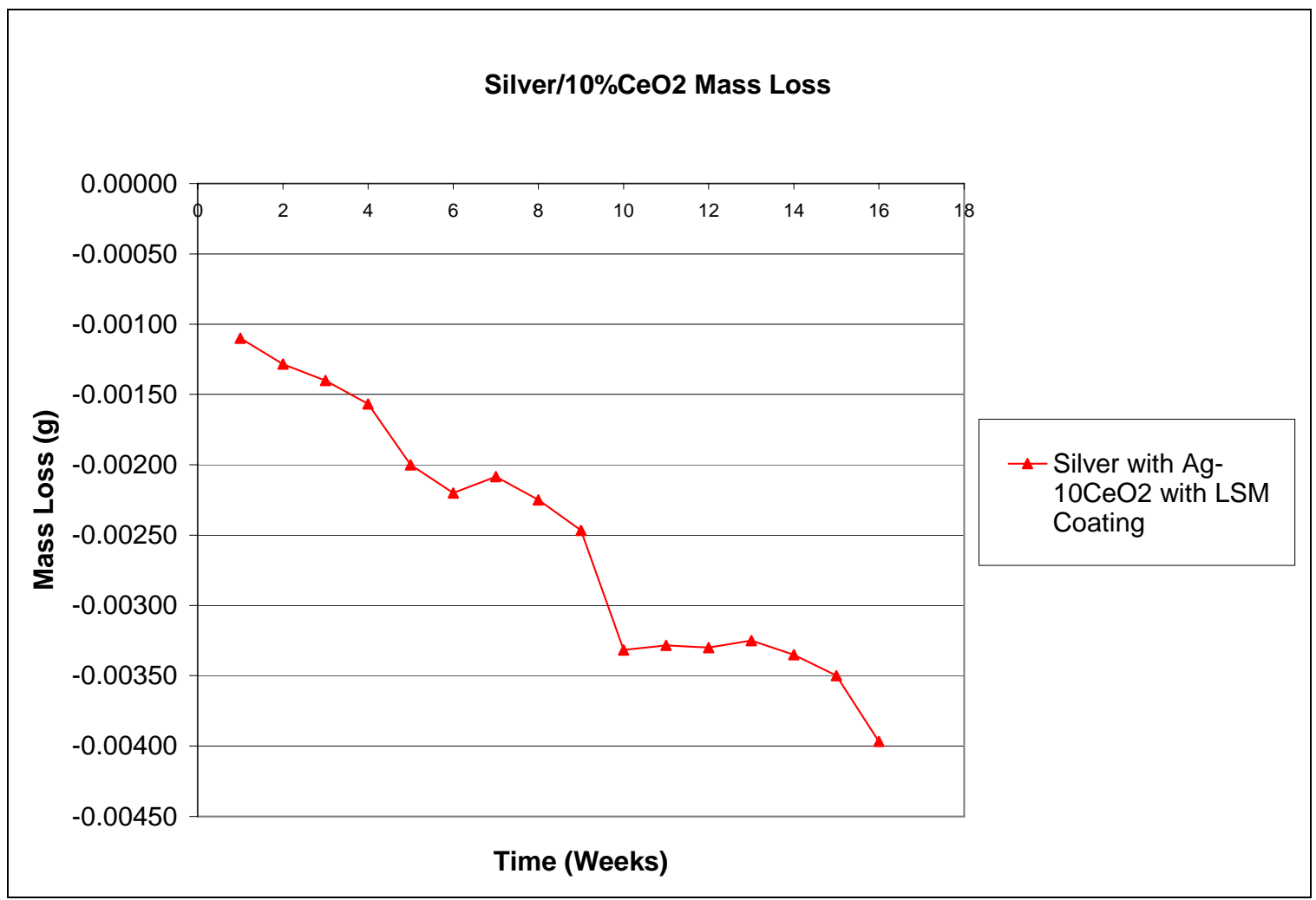

Figure 10 Mass loss of silver/10\%ceria-LSM sample

At week eight, however, a single week of seemingly rapid mass loss occurred, after which the mass reduction rate stabilized again. As with the silver-LSM sample, this provides evidence of significant silver diffusion in the weeks prior to the increase in mass loss. The average weekly mass loss was used to establish the long term mass loss for the silver-ceria-LSM composite material. From this it was found that the sample loses around $0.2479 \mu \mathrm{g}$ per week. This factor is surprising, as it was expected that the ceria component of the silver contact paste would act to slow the evaporation rate of the silver due to ceria's high temperature stability. Over the target life of the fuel cell stack, the silver/10\%ceria-LSM material would lose around $59.50 \mu \mathrm{g}$. 


\subsubsection{Silver-Silver/40\%Ceria-LSM}

The screen printed silver $/ 40 \%$ ceria-LSM samples followed a similar mass reduction trend as the silver/10\%ceria-LSM and the silver-LSM sample, however with the reduction rate being higher.

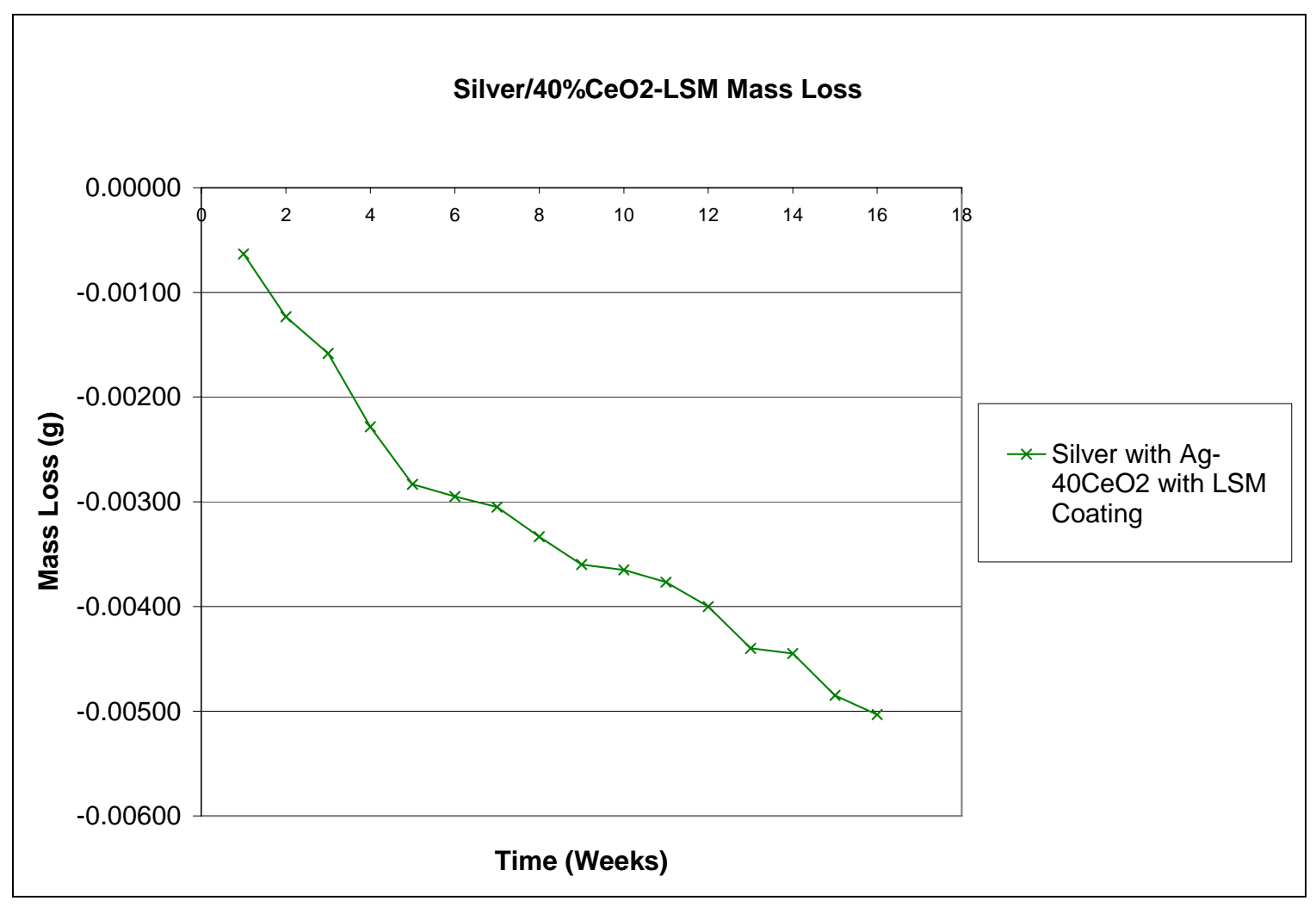

Figure 11 Mass loss of silver $\mathbf{4 0} \%$ ceria-LSM sample

In contrast to the mass loss rate of the other samples, the silver $/ 40 \%$ ceria-LSM experienced a significant mass loss after three weeks. This seems to indicate that the addition of ceria into the silver contact paste increases the diffusivity of silver, as the detected period of non-linear mass loss occurs may occur progressively earlier for the 
sample as the percentage of ceria increases. In order for this to mass loss to occur, the diffusion rate of silver into the LSM layer must have increased as ceria percentage increased. The average mass loss per week of the silver $/ 40 \%$ ceria-LSM sample was $0.3146 \mu \mathrm{g}$ per week. Total cell lifespan mass loss was calculated to be $75.5 \mu \mathrm{g}$.

\subsubsection{Screen Printed Silver}

The screen printed silver sample exhibited a mass loss rate slightly higher than that of the silver-LSM sample, but had superior performance in comparison to the samples including ceria.

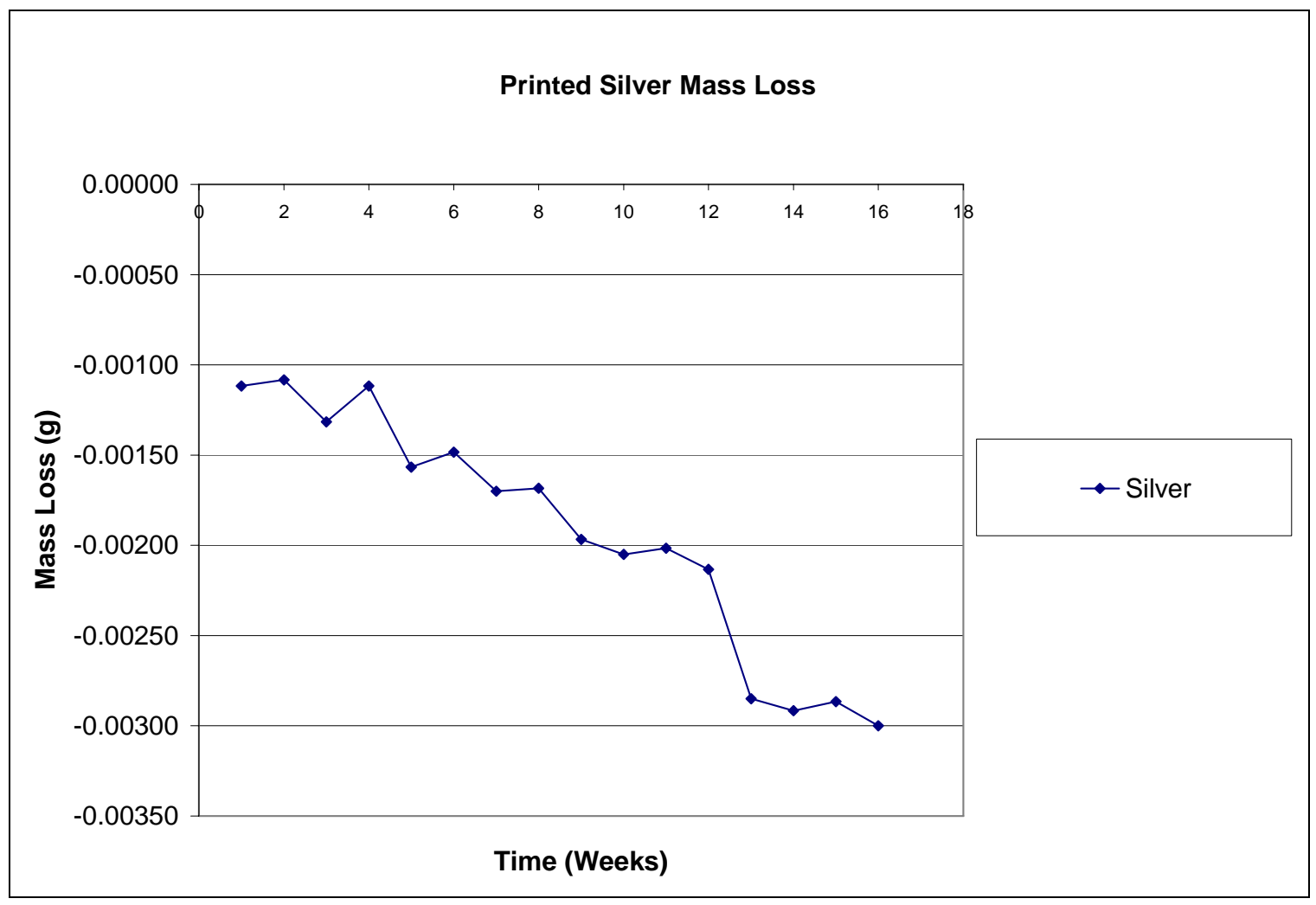

Figure 12 Mass loss of screen printed silver sample 
Of note is the fact that a high mass loss of $1.116 \mu \mathrm{g}$ was detected during the first week of exposure, followed by reductions of $0.0924 \mu \mathrm{g}$ per week, indicating a period of initial instability, followed by the expected stability due to faceting and reorientation of the surface of the silver to achieve low surface-free energy. Pure silver appeared to experience a rapid mass loss rate of $0.7167 \mu \mathrm{g}$ per week for between exposure weeks 12 and 13. This rapid mass loss was visually apparent as well, with the sample clearly losing a great deal of mass radialy. This effect was not noticeable visually with the LSM coated samples, as the silver was coated. Documentation of silver behavior under the LSM coating layers will be discussed later.

The average mass loss rate of the pure silver was $0.1875 \mu \mathrm{g}$ per week. Over the desired lifespan of the cell stack, printed silver would lose $45.00 \mu \mathrm{g}$.

\subsubsection{Silver Foil}

Previously conducted research by Sakacsi et al was made available for comparison purposes. The sample used was a 50 micron thick silver foil, the sample thickness found by Sakacsi et al to have the lowest mass loss rate. This data was collected under the same conditions using the same equipment and set up used for the second tube furnace. 


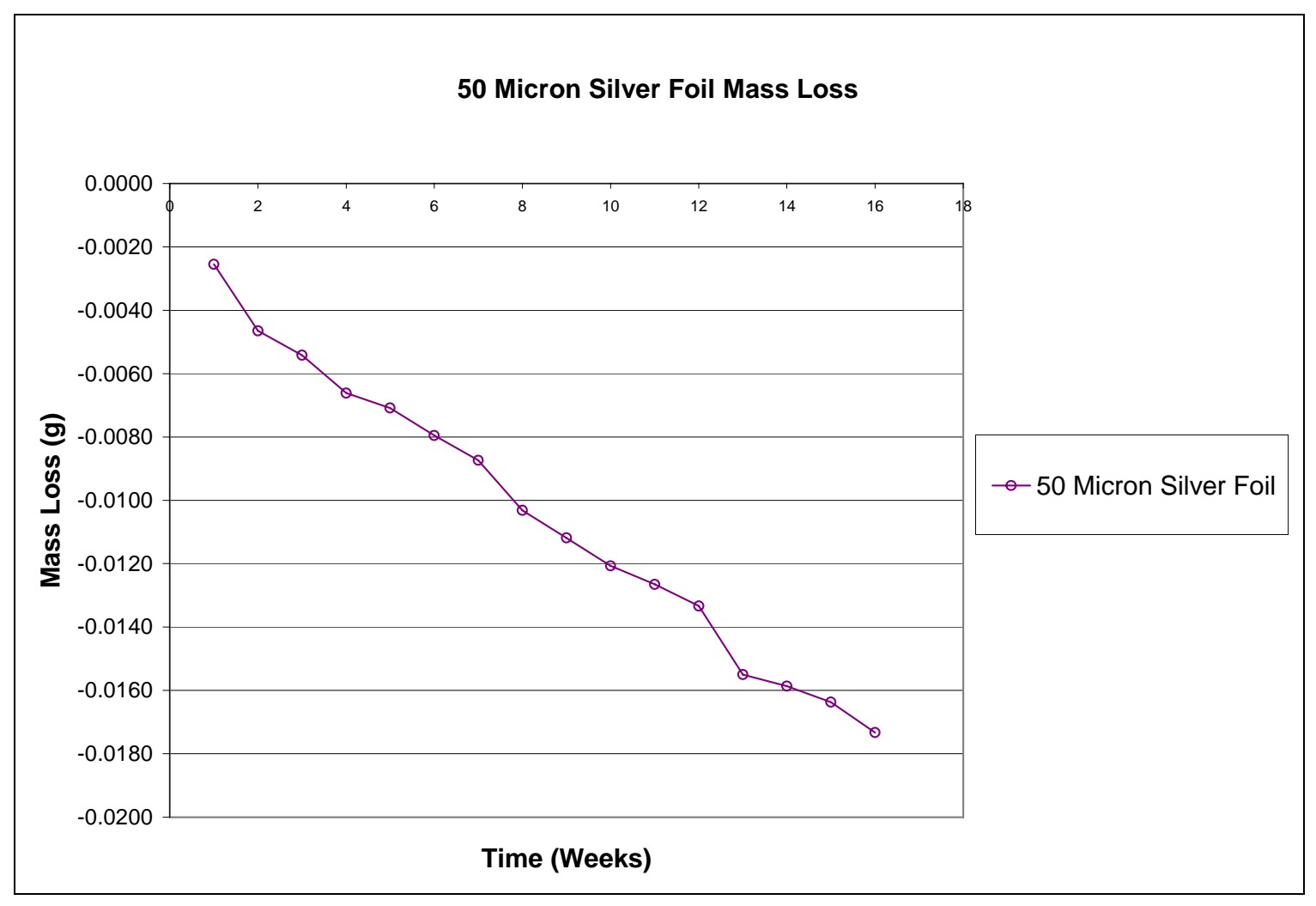

Figure 13 Mass loss of silver foil (50 microns)

From the collected data, the mass loss rate of the silver foil was roughly 1.1083 $\mu \mathrm{g}$ per week. This is approximately $550 \%$ greater than the mass loss rate of the screen printed pure silver, and a $1050 \%$ increase over the weekly reduction rate of the silverLSM sample.

\subsubsection{Mass Loss Rate Comparison}

A comparison of the samples accumulated mass loss is presented in the figure below. 


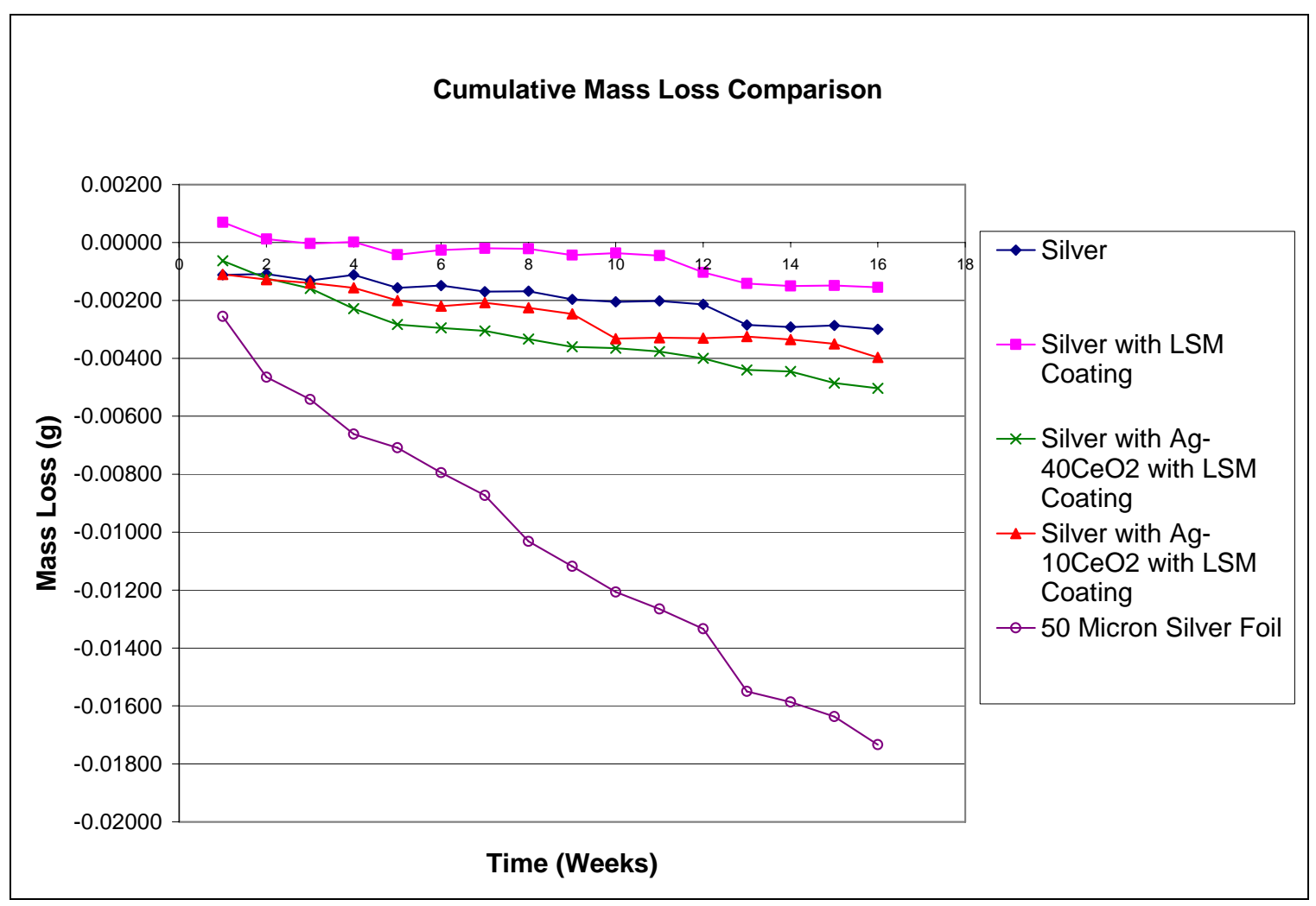

Figure 14 Cumulative mass loss of all samples

From this figure it is clear that the Ag-LSM sample lose mass at a lower rate than the screen printed pure silver, the silver foil, and the silver ceria composite samples. It is also clear that all samples appear to experience a period of instability and non linear mass loss, usually between weeks 9 and 12. The mass loss rate of the screen printed samples was lower than that of the 50 micron silver foil. The non-linear mass loss rates of the samples may indicate that silver diffusion is occurring in the screen printed composite samples.

It was hoped that the inclusion of ceria would inhibit the evaporation of silver, while providing a stable, conductive metallic-oxide to help hold the silver in place, however it appears that the inclusion of ceria in the samples serves to increase the diffusion rate of the silver particles. This increased diffusion results in greater exposure 
of silver and an increase in the evaporative loss. The mass loss rate of the silver $/ 40 \%$ ceria samples is comparable to that of the 50 micron silver foil.

The non-linear mass loss in the screen printed silver and the silver foil samples seems to indicate that the non-linear mass loss of the silver is not dependent upon the rate of silver diffusion through the LSM layer. However, the silver-LSM sample experienced non-linear mass loss at a reduced rate, corresponding to roughly $50 \%$ of the higher mass loss rate of the printed silver specimen. The data suggests that silver, regardless of whether it is a deposited particulate or a foil, experiences mass loss in a non-uniform fashion, with periods of increased mass loss occurring, the timing of which seems to be dependent upon the material characteristics and composition.

All samples were exposed at the same time, with flow rates equal in each tube. This removes the possibility of a systemic error caused by a sudden increase in air flow rate or temperature, as the sharp increases in mass loss would occur at the same time. It also is clear that the coating of the silver with LSM reduces the mass lose rate of the silver from the sample. The samples containing ceria exhibit an increased mass loss rate, indicated by the period of accelerated mass loss occurring at weeks nine and three.

Scanning electron microscopy of the surface of the samples, as well as from fracture surfaces of the sample was conducted to determine the possible causes of the mass loss results. 


\section{CHAPTER 4: SEM}

\subsection{PARTICLE ANALYSIS}

The powders used in this project were analyzed using scanning electron microscopy to determine their morphology prior to being made into ink. The process used for analysis was placing a small amount of the powder material on top of carbon tape on the SEM stage.

The powders used in this study consisted of micron or sub-micron particles clumped together into larger balls. These larger balls will be broken up during the manufacturing of the inks used in the screen printing process. This process involves grinding the powder on ceramic rollers while the terpiniol binder is added. The rollers serve two purposes. First, they break up the larger balls of the powder, ensuring that the sub-micron sized particles are suspended in the binder. Second, the rollers serve to ensure even distribution of the particles throughout the ink, so that uniform printing layers can be deposited.

\subsubsection{Silver Powder}

The silver powder used in this study consisted of very small particles that clump together to form larger particles. It is clear that the particles have an aggregate composition. As the larger balls would be broken during the ink mixing process, the smaller particles that make up the larger powder particles are what will actually be studied in this investigation. 

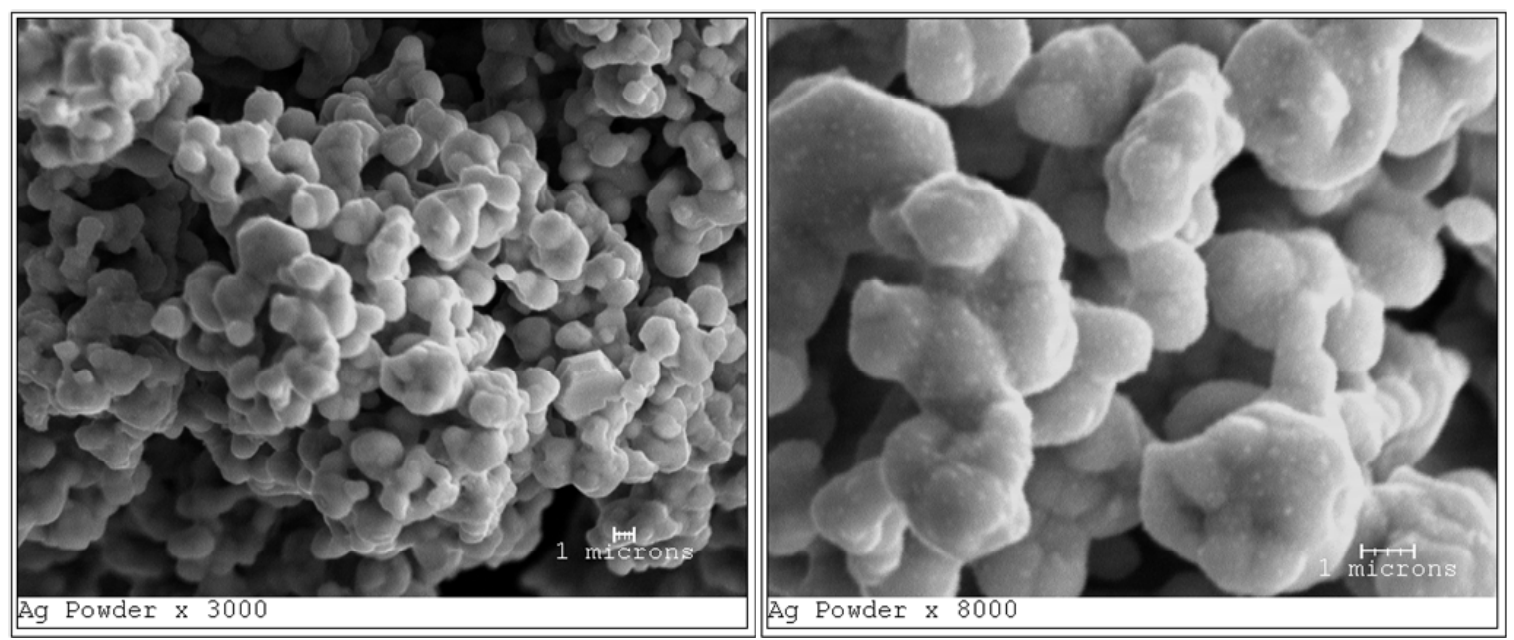

Figure 15 Surface of silver powder showing small silver particles

The smaller particles of silver were found to be $1-3 \mu \mathrm{m}$ in diameter.

\subsubsection{LSM Powder}

LSM powder was found to consist of spherical particles of greatly varying sizes.

While most particles were found to be $50 \mu \mathrm{m}$ in diameter, larger particles of $100 \mu \mathrm{m}$ diameter were measured. These particles had an overall spherical nature, however upon closer inspection, the surface of the LSM powder is spongy in nature, and is made up of very small compacted particles. The compacted particles have an average diameter of $0.75-1 \mu \mathrm{m}$, and are angular in shape. 


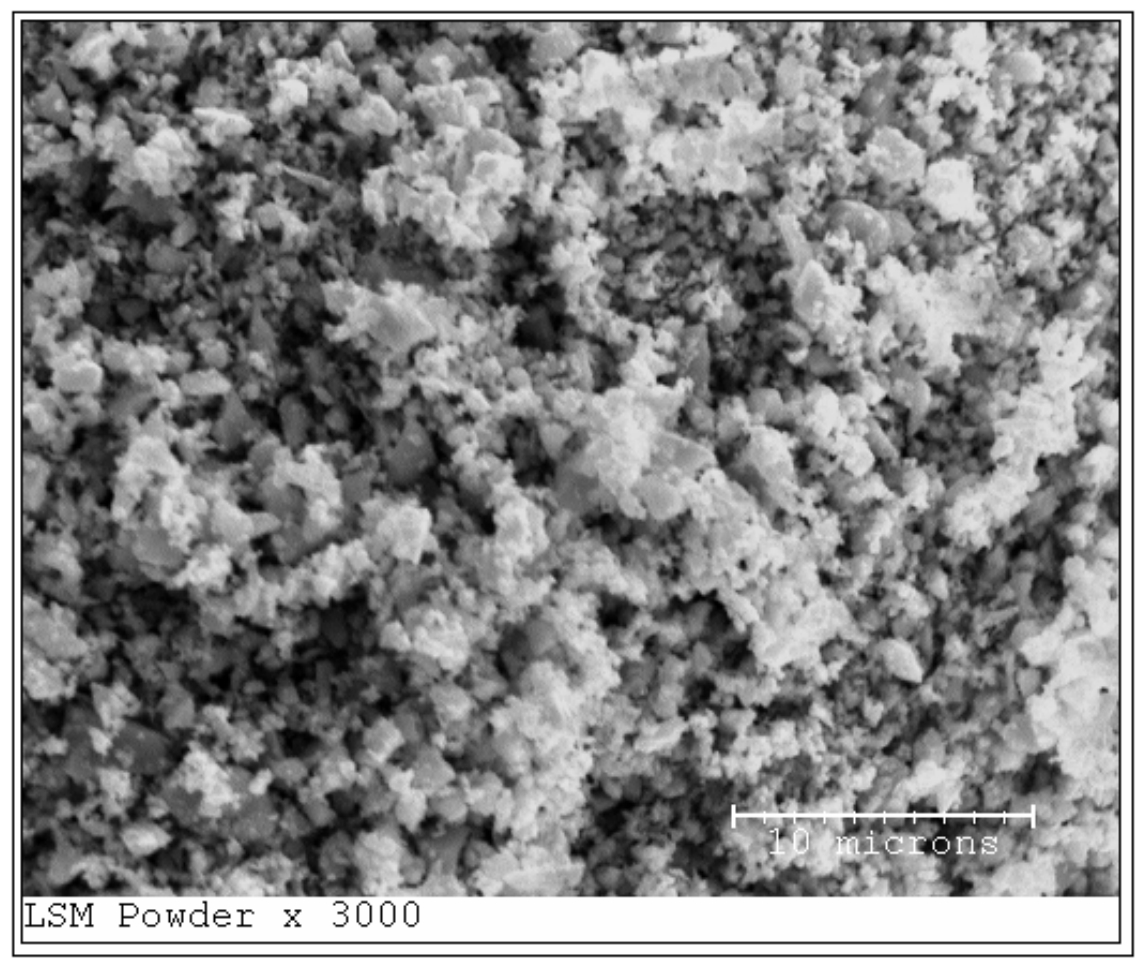

Figure 16 Surface of LSM powder showing smaller LSM particles

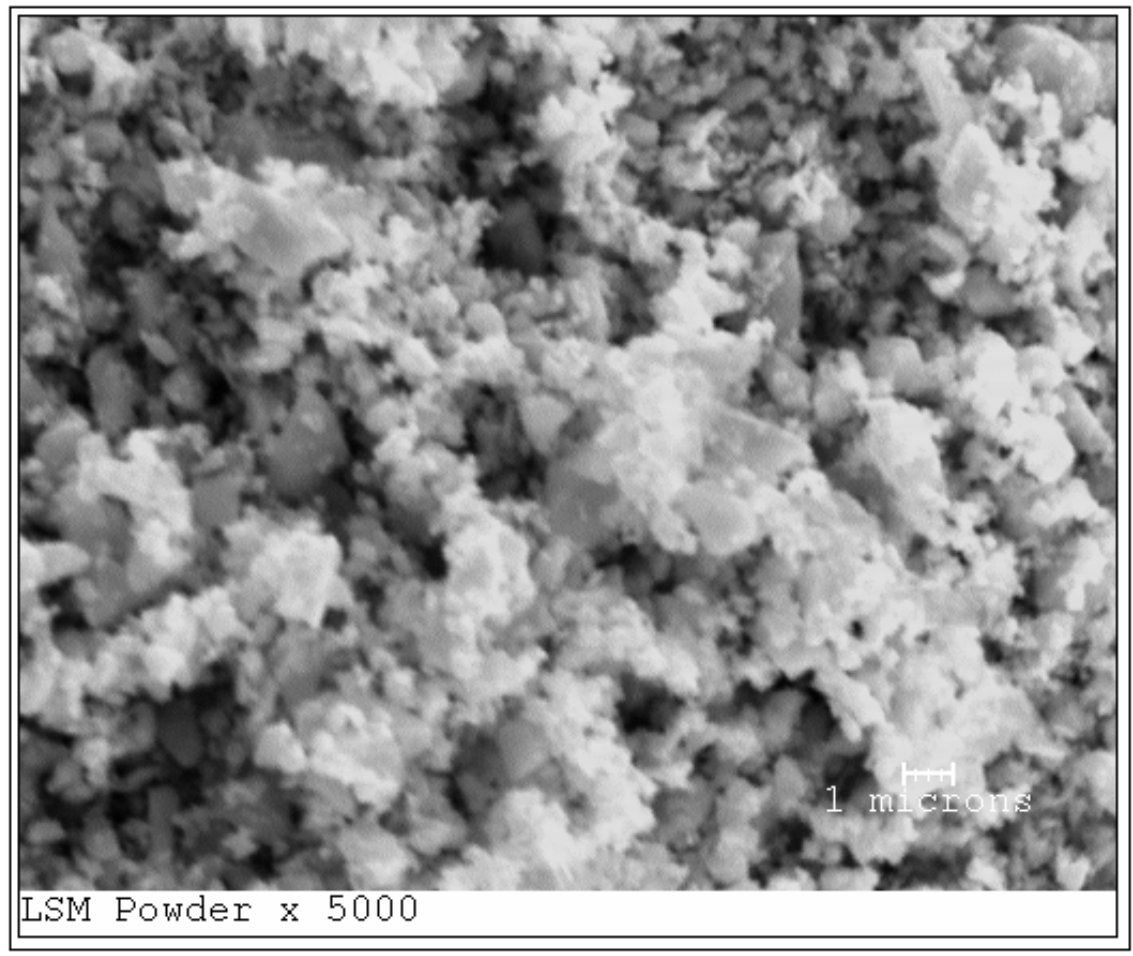

Figure 17 Angular nature of LSM particles 


\subsubsection{Silver-10\%Ceria Composite Powder}

There was a great deal of variation in the silver-10ceria particle size. The powder, a mixture produced through a glycene nitrate process, had larger spherical particles of silver, ranging from $25 \mu \mathrm{m}$ to $50 \mu \mathrm{m}$ in diameter. Mixed in with the silver were ceria particles which were irregular and spongy in nature. The ceria also showed a significant variation in dimension, with some particles being independent and made only of ceria, but with large amounts of ceria coating the surface of each silver particle. The extent to which the ceria has bonded to the surface of the larger silver particles is illustrated in the following figure. The ceria attachment occurs in clumps on the surface of the silver, but is not evenly distributed.

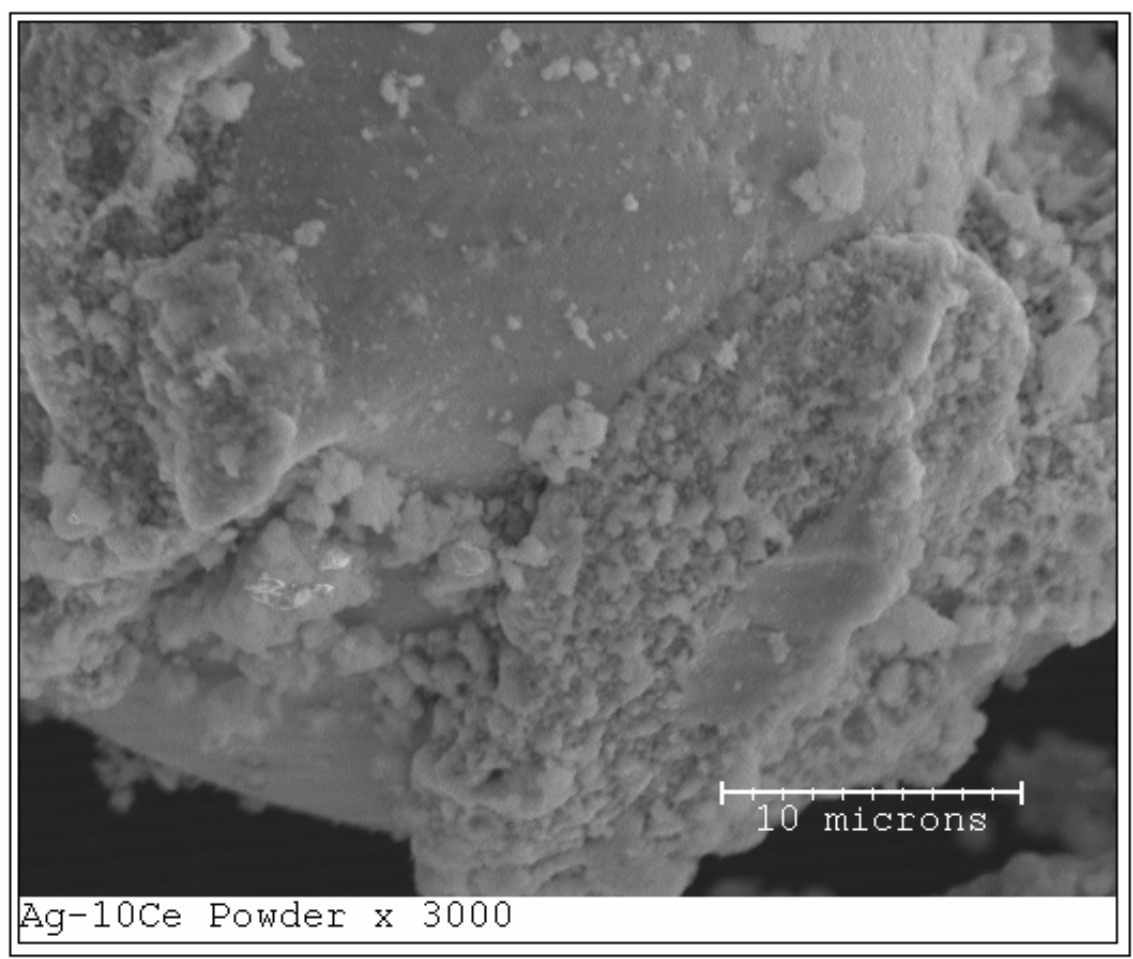

Figure 18 Surface of spherical silver particles showing ceria adhesion 


\subsubsection{Silver-40\%Ceria Powder}

The silver- $40 \%$ ceria powder consisted of silver particles of the same size as the $10 \%$ ceria powder. However, since the weight percent of silver is less than in the $10 \%$ ceria powder, the number of silver particles is reduced. Also, the surface of each silver particle is almost completely coated with ceria, as show in the figure below.

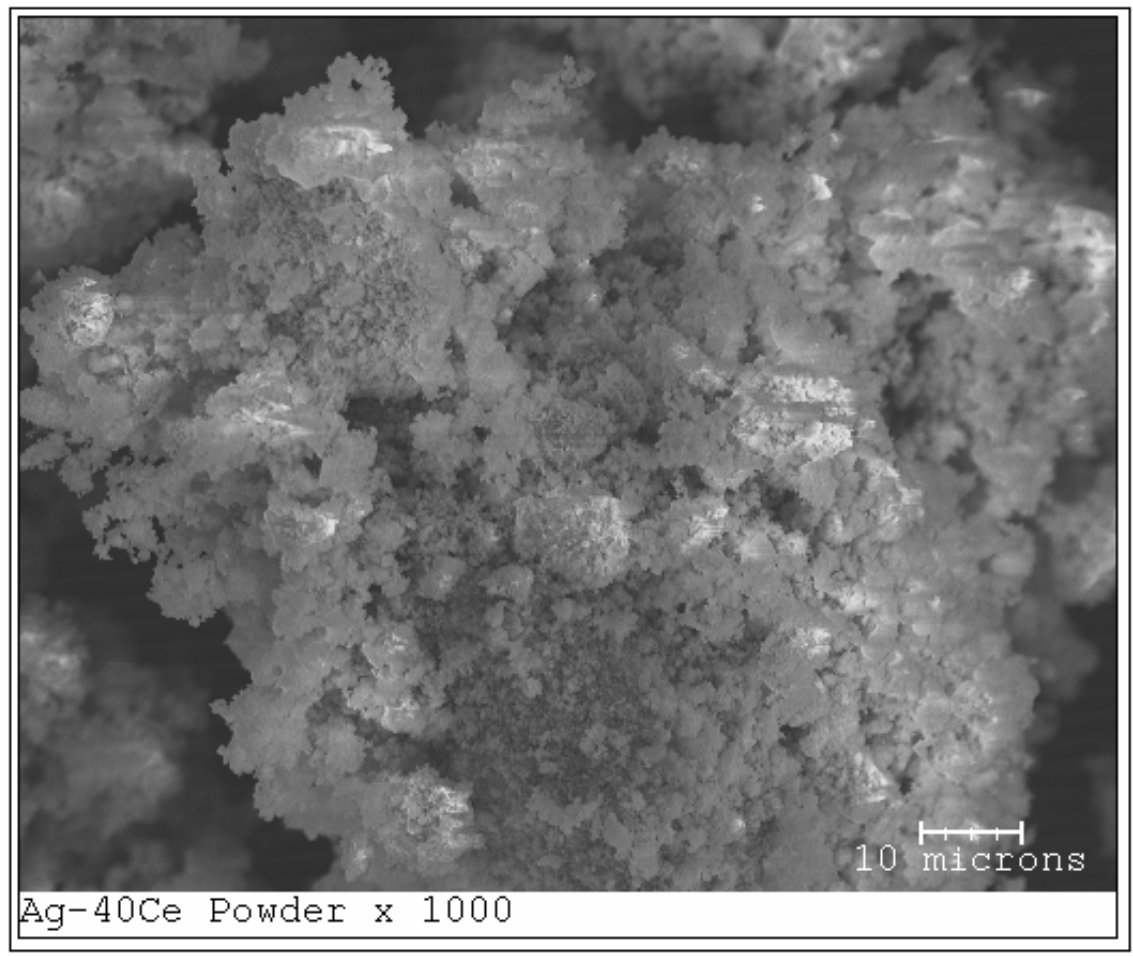

Figure 19 Silver-40Ceria particles showing ceria adhesion

\subsection{SAMPLE ANALYSIS}

\subsubsection{Silver-LSM}

To monitor the evolution of the sample microstructure during high temperature exposure, scanning-electron microscopy (SEM) was used. Images were made after binder 
burnout, then weekly for the first five weeks. After the fifth week, micrographs were made bi-monthly for the remainder of the experiment time line.

Images were taken of the sample surfaces after binder burnout, but prior to exposure to the air flow. Pre exposure imagery of the silver-LSM surface is shown below.

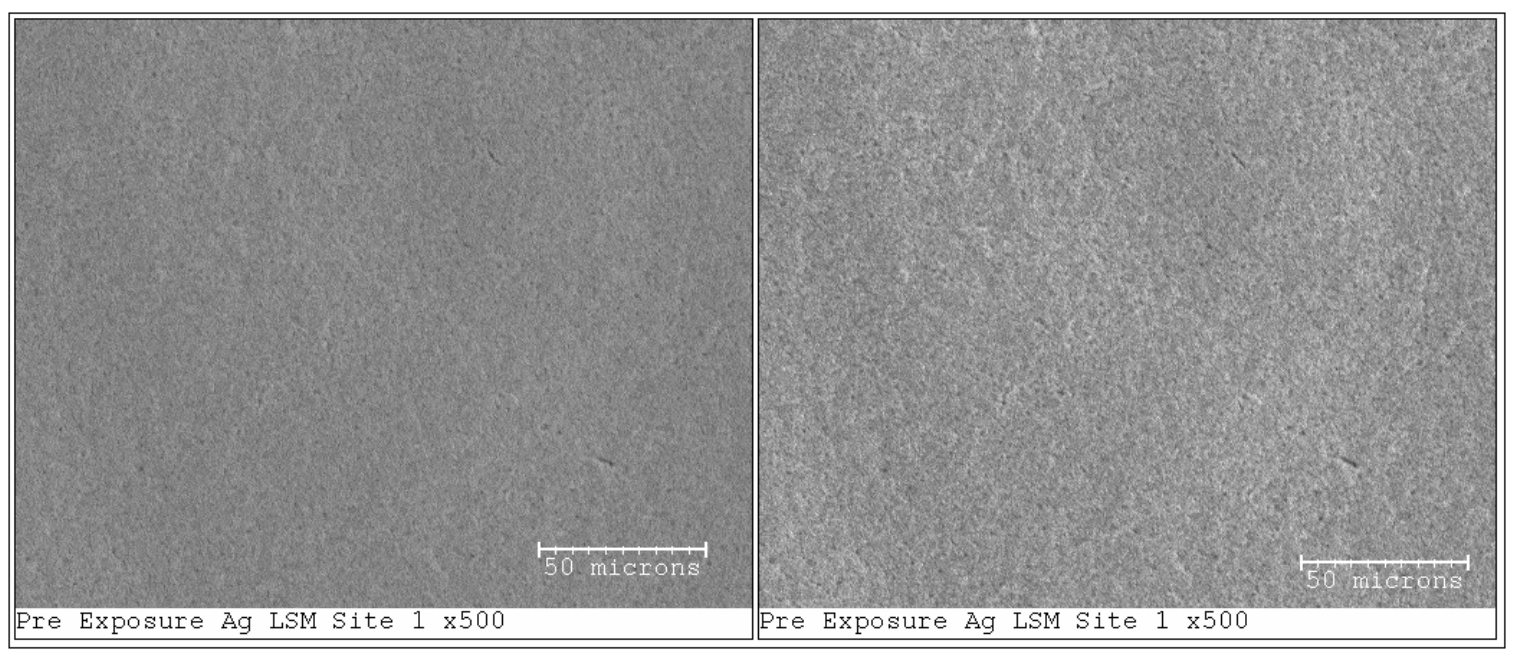

Figure 20 SEM micrograph and backscatter of surface of pre-exposure silver-LSM sample

Backscatter images of the surface are shown at right in the figure above. In backscatter imagery, elements of heavier atomic mass are shown lighter in color, while lighter elements appear darker. The image on the right in Figure 20 indicates that the majority of the sample is LSM. This is further validated by the x-ray spectrum of the surface. 


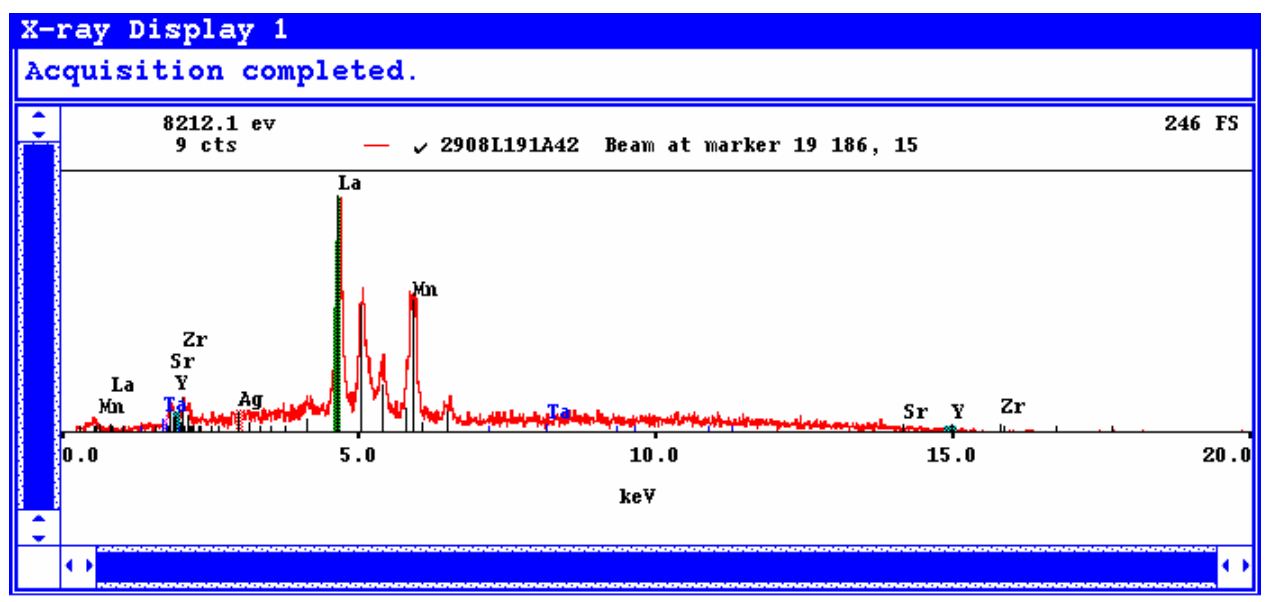

Figure $21 \mathrm{X}$-ray spectrum of pre-exposure silver-LSM surface

It can be seen in the x-ray spectrum of the surface that the surface is primarily covered with lanthanum and manganite, with a small component of strontium. The large peak around $5.0 \mathrm{keV}$ is the oxide peak for lanthanum. From this it is clear that the surface is coated with LSM, and that while small amounts of silver are detectable, the LSM coating layer is only $\sim 25 \mu \mathrm{m}$ in thickness. As this is near the penetration depth of the $\mathrm{x}-$ ray no silver diffusion has occurred into the LSM after binder burnout ${ }^{26}$.

After one week of exposure, the surface of the Ag-LSM sample appears to have changed little. The image below shows the surface of the Ag-LSM sample in the same location as presented in Figure 22 


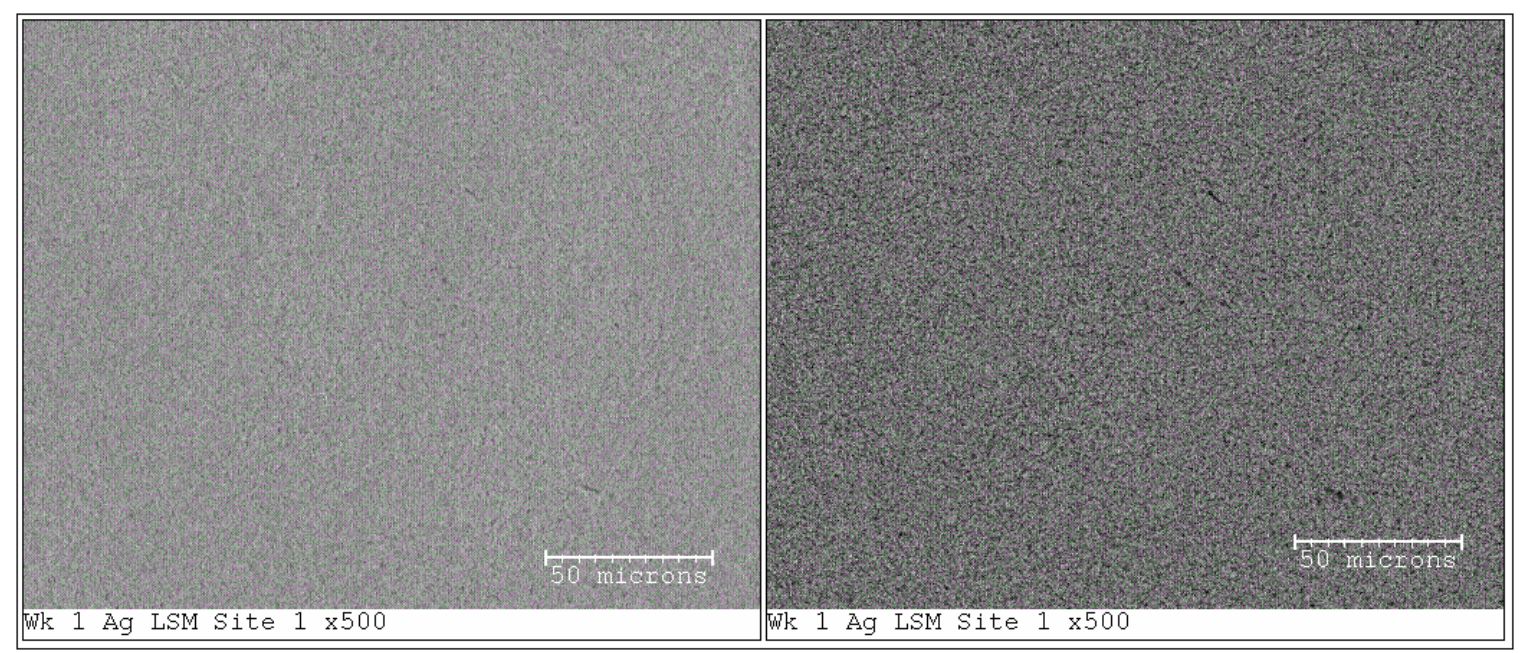

Figure 22 SEM micrograph and backscatter of silver-LSM surface (1-week exposure)

From the backscatter image, no silver is apparent at the surface of the sample, with the surface of the sample remaining coated in LSM.

After two weeks of exposure, the surface of the sample shows no agglomeration of the LSM particles. This was to be expected from previous data documenting the stability of LSM at high temperatures and when exposed to varying flow rates.

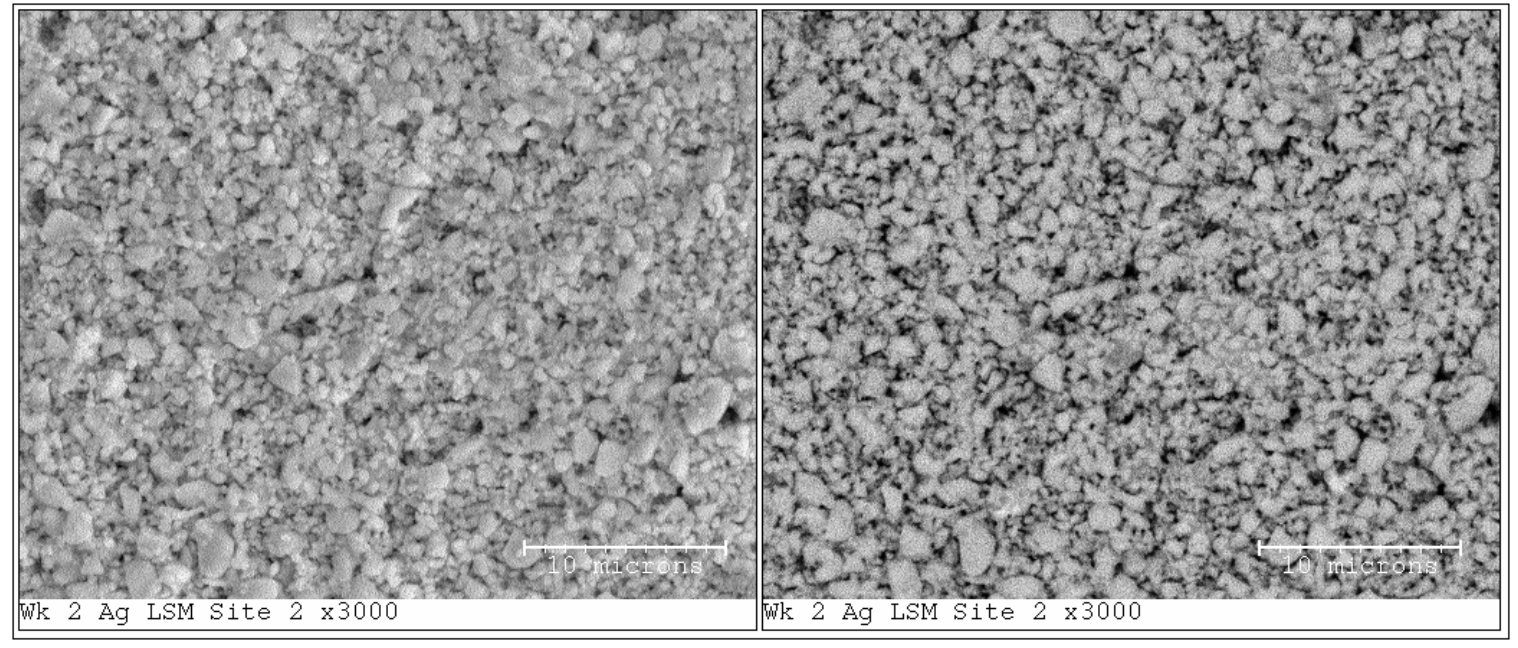

Figure 23 SEM micrograph and backscatter of silver-LSM surface (2-weeks exposure) 
The largest particles appearing on the surface are around 1-2 $\mu \mathrm{m}$ in diameter, with the average particle size being between 0.5 and $1 \mu \mathrm{m}$. Backscatter imagery again shows no silver on the surface. In the backscatter image on the right side Figure 23, the surface is shown to be of singular composition. The dark areas that are clearly visible are actually gaps in the surface texture between particles.

At week three, there is again little change in the surface of the sample

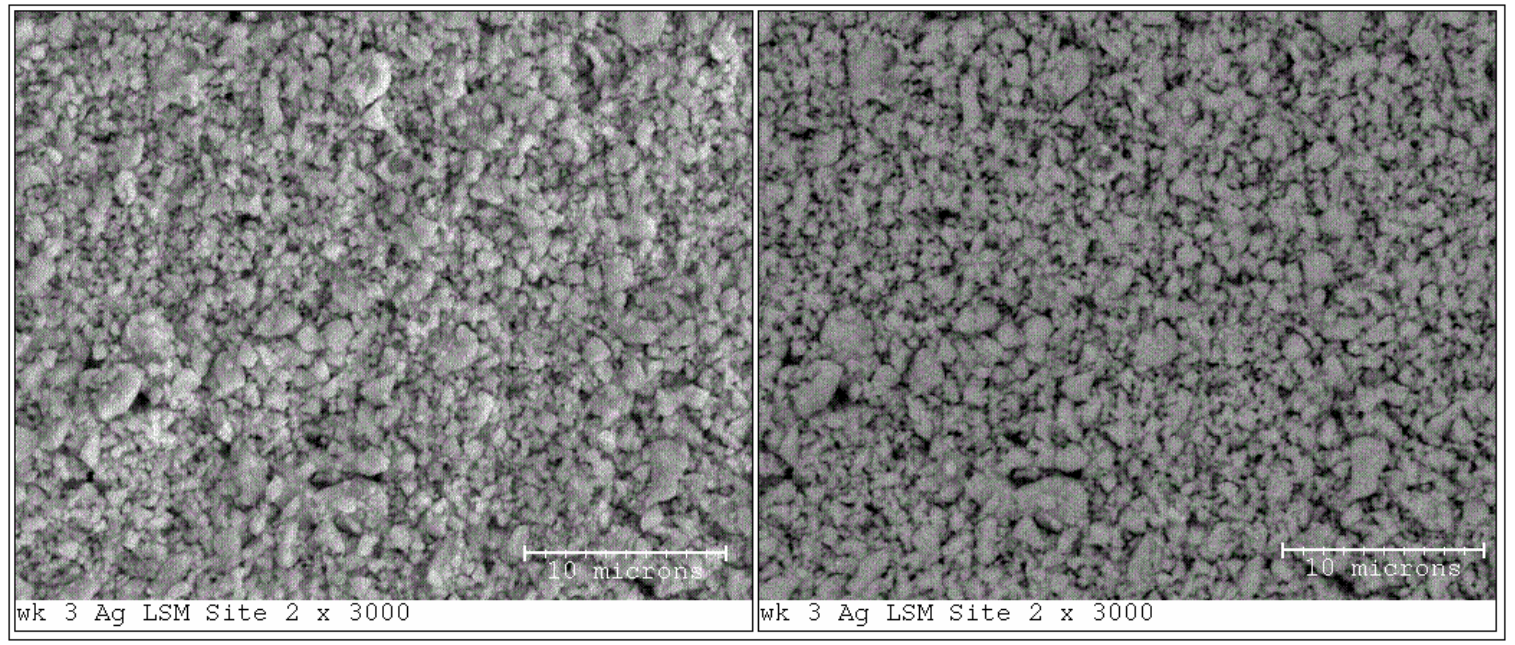

Figure 24 SEM micrograph and backscatter of silver-LSM surface (3-week exposure)

The LSM particles on the surface indicate no agglomeration. This is apparent by the many LSM particles on the surface of diameters lower than $0.5 \mu \mathrm{m}$ that are visible from week to week in the above figures, which while slightly offset, are taken at the same location.

The x-ray spectrum of the above location indicates an increase in detectable silver just below the surface. As x-ray spectroscopy has a penetration depth into the sample, an increase in detectable silver indicates silver migration toward the free surface of the sample. This would result in an increase in the detectable silver. This increase over the pre-exposure spectrum is shown below. 


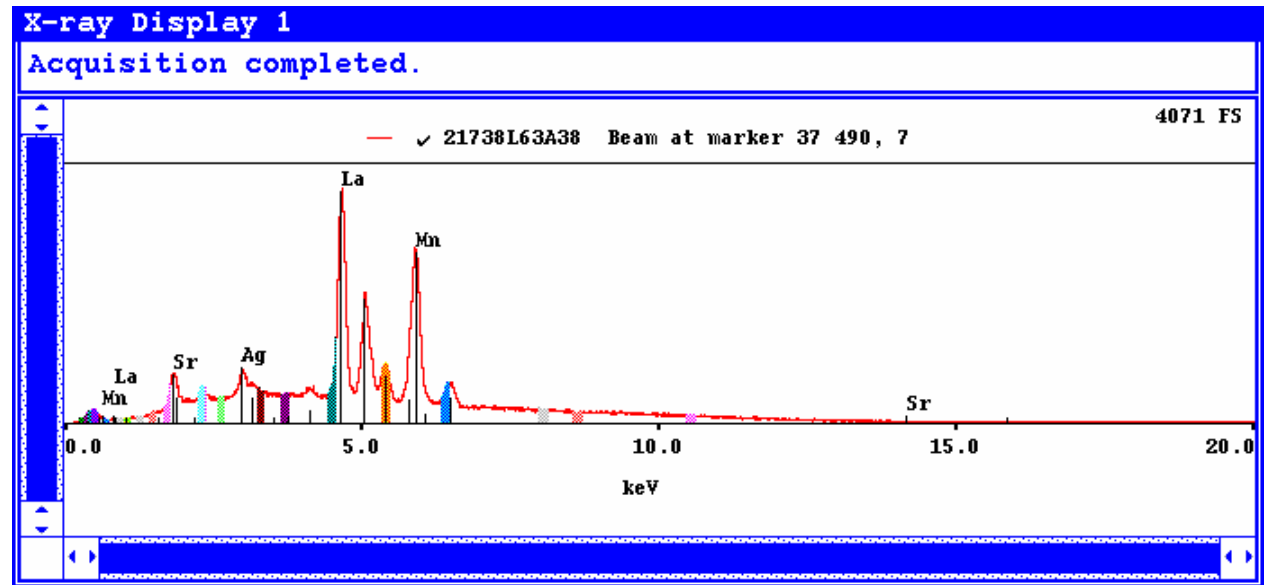

Figure 25 X-ray spectrum of silver-LSM surface after 3 weeks exposure showing increase in detected silver

The increase in the silver detected by X-ray analysis serves as evidence that silver is migrating into the LSM coating layer.

After twelve weeks no agglomeration of the LSM particles on the surface of the Ag-LSM sample was evident, as shown in Figure 26.

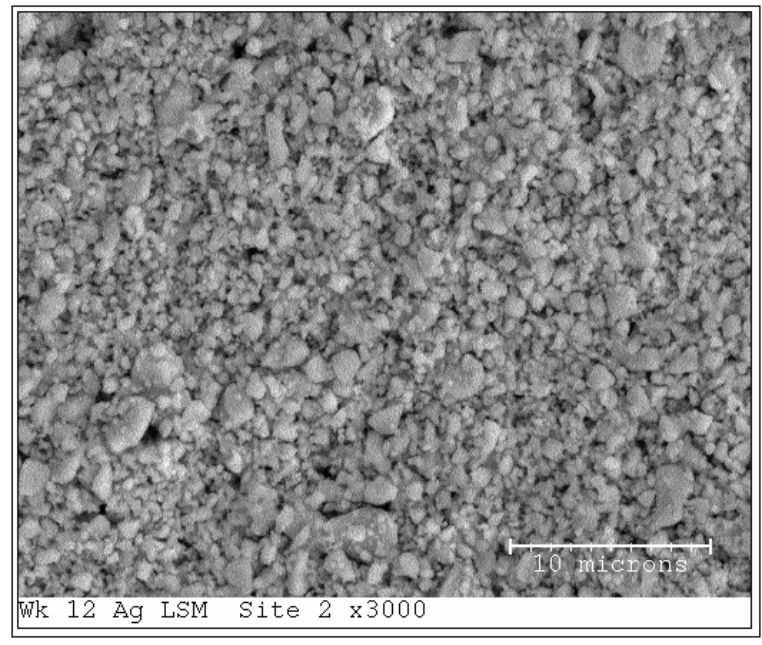

Figure 26 SEM micrograph of silver-LSM surface (12-weeks exposure) 
This result was expected and verified the stability of LSM when exposed to high temperature flow. The LSM coating layer was noticed to be highly susceptible to flaking and spallation due to thermal expansion during rapid temperature changes. Great care was taken to ensure slow heating and cooling occurred when then samples were reinserted in the tube furnace.

\subsubsection{Silver-LSM Fracture Surface Analysis}

Fracture-surface analysis of silver-LSM samples was conducted on an unexposed silver-LSM sample and on specimens at numerous periods of exposure in an effort to determine the behavior of the bulk of the silver covered by the LSM coating layer. As noted previously, X-ray analysis has indicated that silver diffuses into the LSM.

Sample cross sections were prepared by scoring the YSZ substrate and breaking the samples in half. Freezing of the samples in liquid nitrogen was tried, by this dissolved some of the sample, as well as increased spallation of the LSM coating of the sample due to thermal expansion. Fracture of the sample using this method limited distortion or smearing of the sample that could occur using other methods. However, care had to be taken to prevent LSM flaking around the fracture site when the sample was broken.

Pre-exposure fracture analysis clearly shows the printed layers on the substrate. The silver layer presented difficulty in obtaining a smooth fracture surface upon breaking. Unlike the LSM layer, silver tended to have sections of the layer remain with either side of the broken sample, resulting in a jagged topography to the silver layer in the sample. 


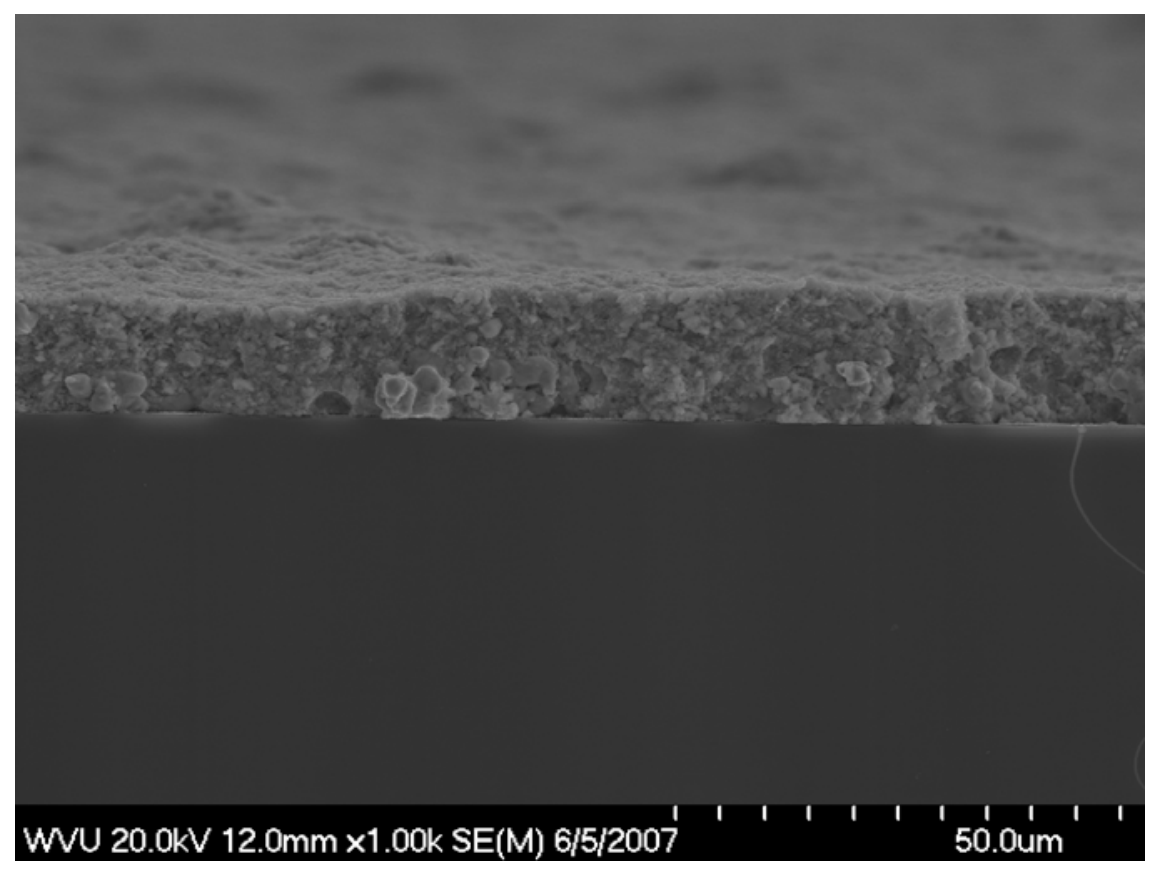

Figure 27 SEM micrograph of pre-exposed silver-LSM cross-section

X-ray mapping of the surface clearly indicated the location of the silver and LSM layers.

The silver layer is shown as a series of clumps in the X-ray map because of the jagged nature of the silver layer fracture.
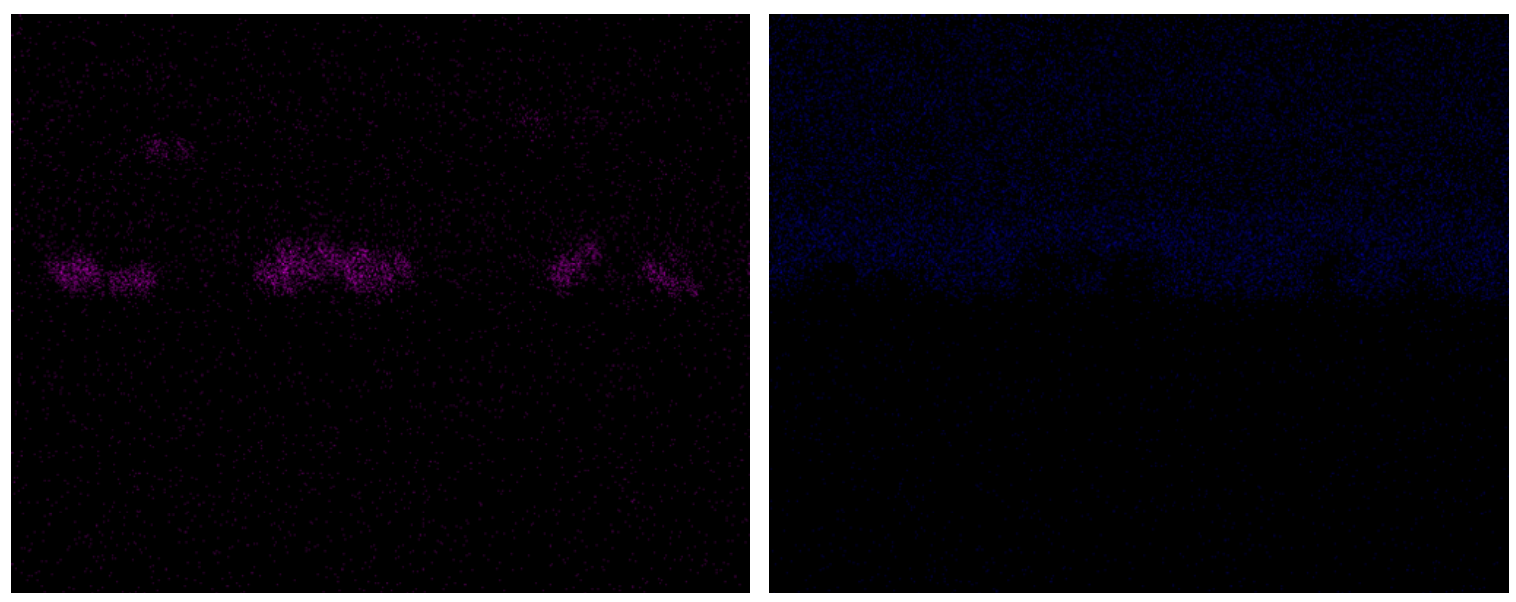

Figure 28 X-ray map of the pre-exposure silver-LSM surface showing, left to right, silver and lanthanum 
The x-ray map, overlaid on the micrograph of the fracture surface reveals the location of the raised areas of the silver layer, which would have more silver detectable.

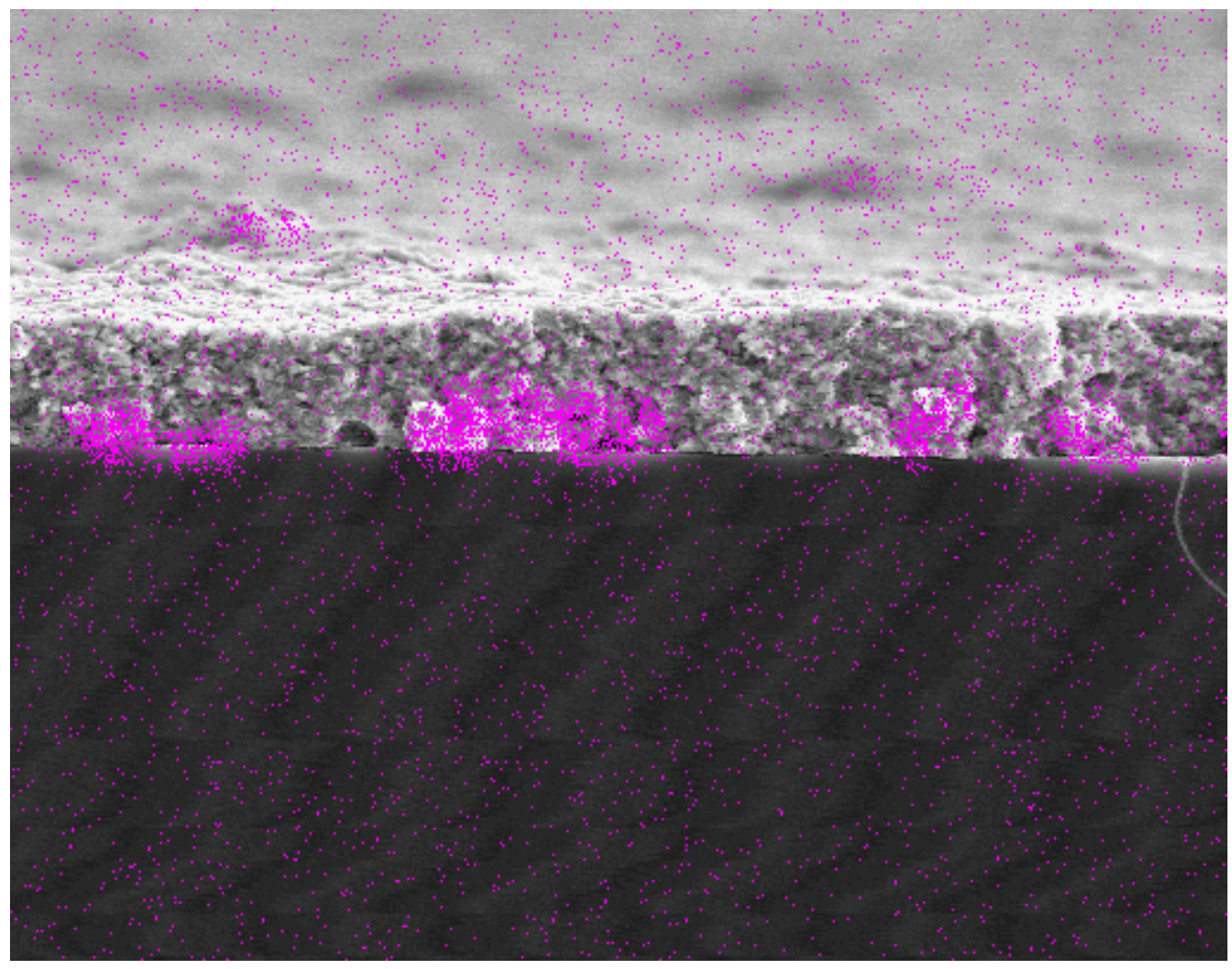

Figure 29 X-ray mapping overlay on the pre-exposure silver-LSM cross section

Spot analysis taken in one of the gaps in the detected silver layer reveals that silver is there in high levels. The spot analysis indicates detected quantities of LSM, but this is due to the fact that the spot analysis was done in a hole on the surface and that more emitted electrons will strike the side of the hole, which is LSM, than the bottom of the hole where the silver is located. 

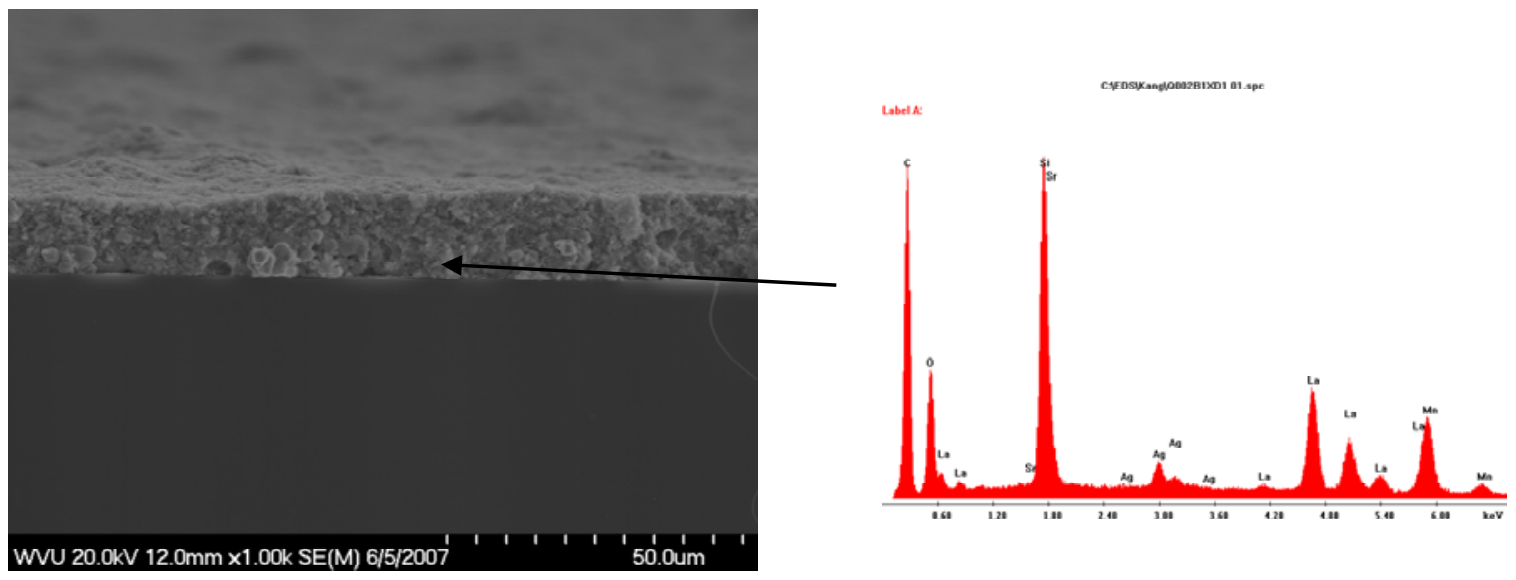

Figure 30 SEM micrograph and x-ray spectrum of pre-exposure silver-LSM cross section

From the X-ray mapping of the sample, it is evident that no diffusion of silver into the LSM coating has occurred. Also, it the silver layer appears to be of uniform thickness across the sample.

In the sample broken after two weeks of exposure, shown below, the composition of the sample is visible as one moves from the substrate, at bottom, to the silver layer in the middle, to LSM at the top of the sample. The free surface of the sample is visible at top. 

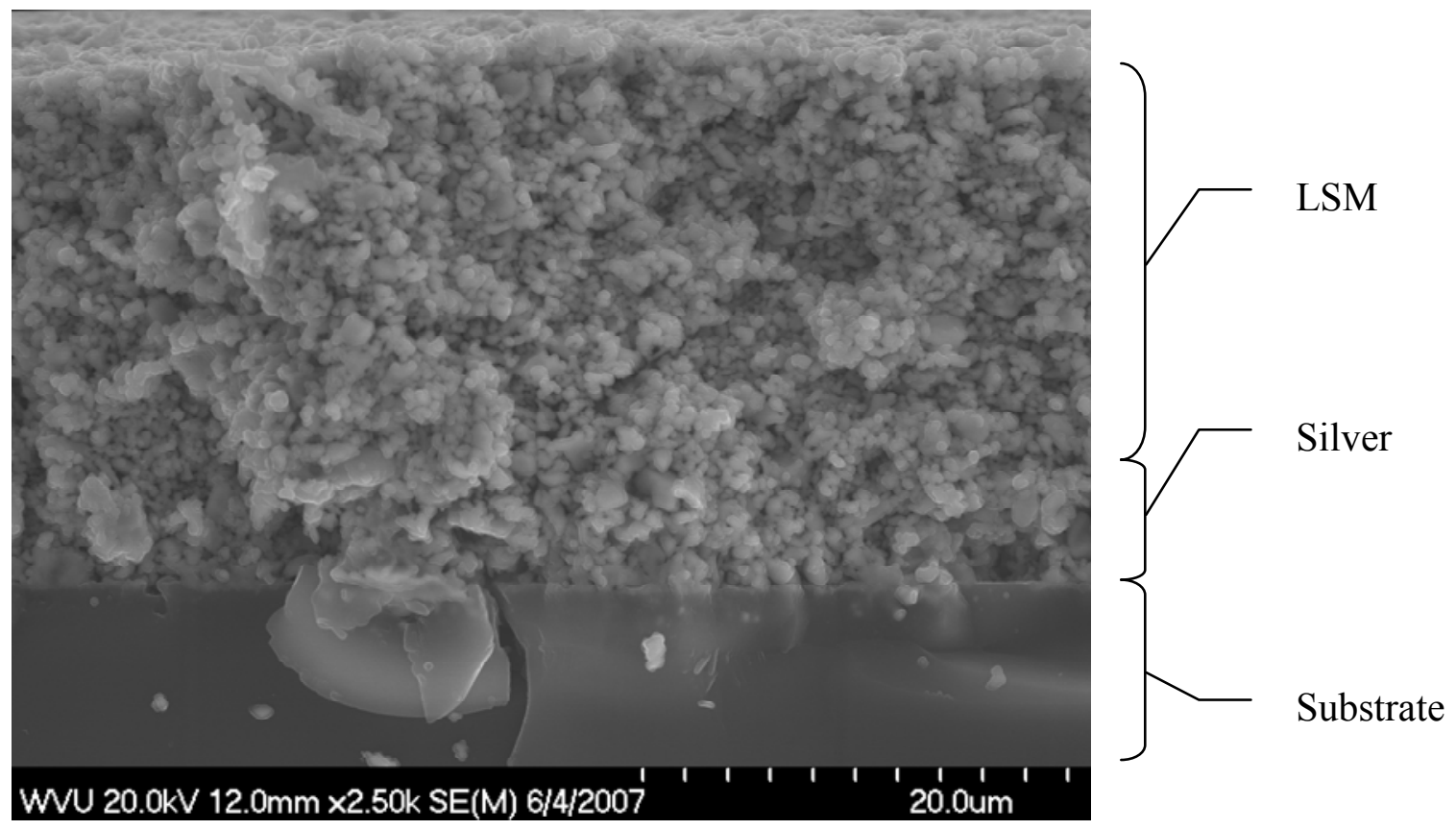

Figure 31 Location of layers within the silver-LSM sample (2-week exposure)

To document the composition of the sample, X-ray analysis was used. The fracture surface is too rough to use backscatter imaging. This same rough surface makes precise identification of individual elements difficult.

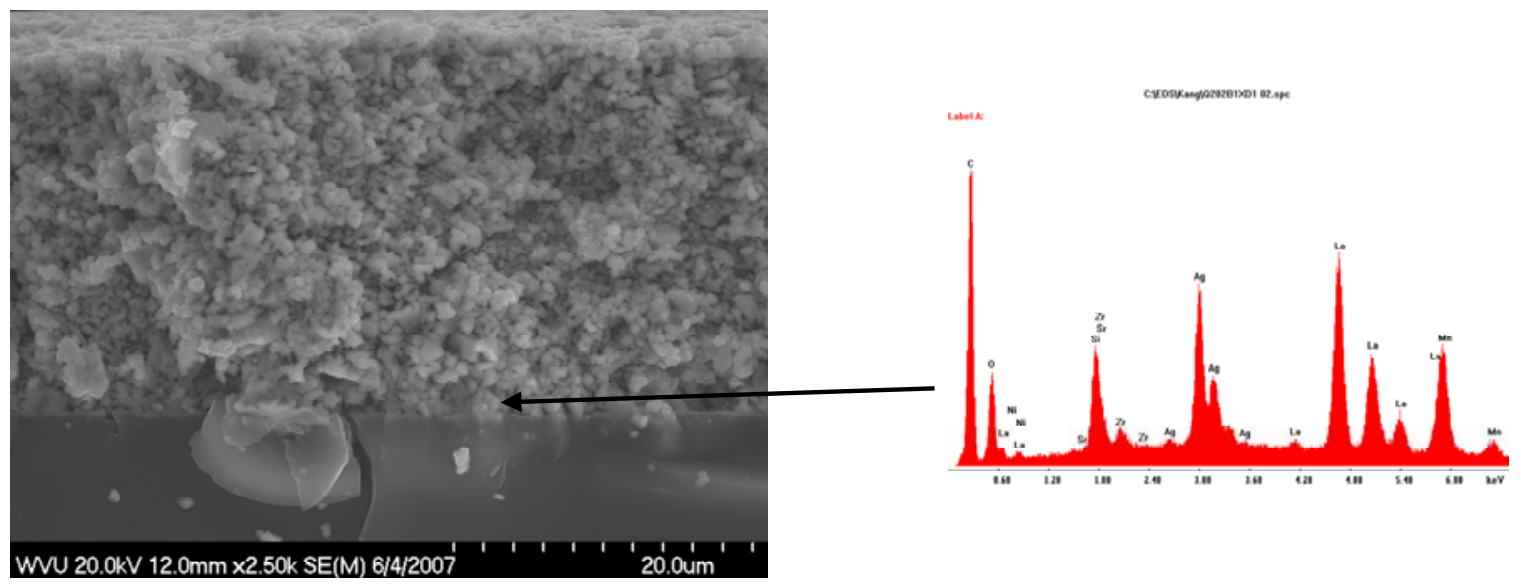

Figure 32 SEM micrograph of silver-LSM cross section with spot analysis of silver layer (2-weeks exposure) 
However, a comparison of the spotlight analysis of the particle shown in the figure above with the X-ray spectrum of the entire surface indicates that the silver content of the region around the particle indicated in the figure above contains much higher quantities of silver than the rest of the sample.

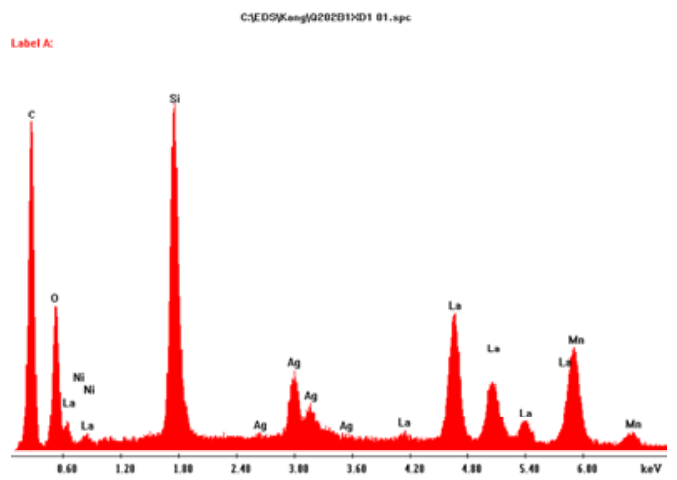

X-ray Spectrum of Entire Surface

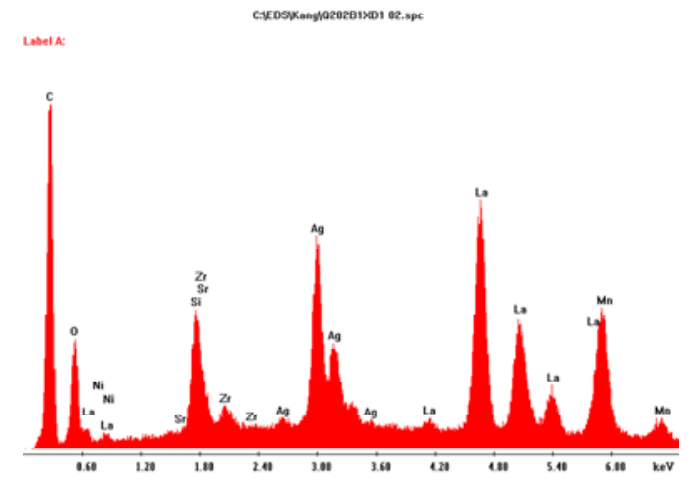

X-ray Spectrum of Silver Region

Figure 33 Comparison of $x$-ray spectrum of entire surface vs. that of silver region (2-weeks exposure)

The large carbon peaks in the both spectra is due to the carbon coating of the samples, while the oxygen peaks is due to oxidation of LSM.

X-ray mapping of the fracture surface also indicated that after two weeks of exposure silver remained largely in its original location. Some migration of silver is evident but appears to occur faster in localized areas after two weeks, as opposed to occurring evenly throughout the entire silver layer. This may be related to a higher localized porosity of the samples LSM layer. 

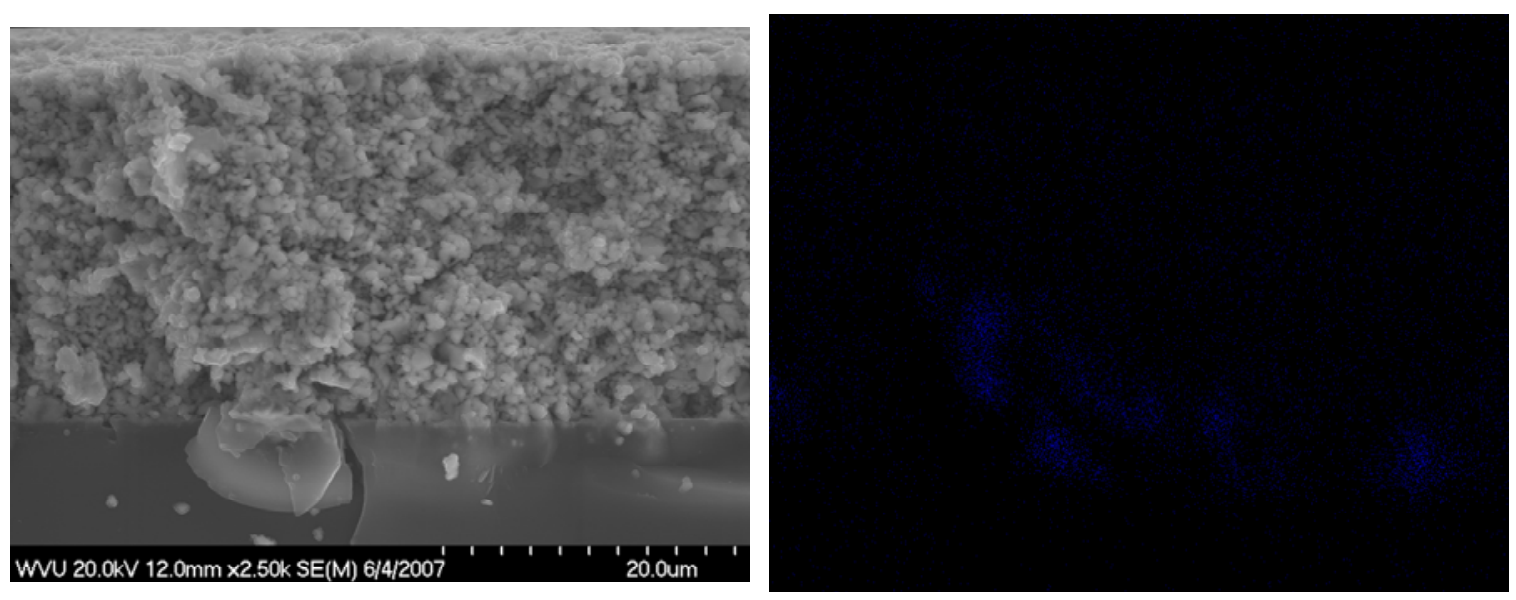

Figure 34 SEM micrograph and x-ray map of silver in silver-LSM cross-section (2-weeks exposure)

The location and migration of silver is clearer when the X-ray map is overlaid on the micrograph of the fracture profile. It should be noted that some image drift occurred during the creation of the silver mapping.

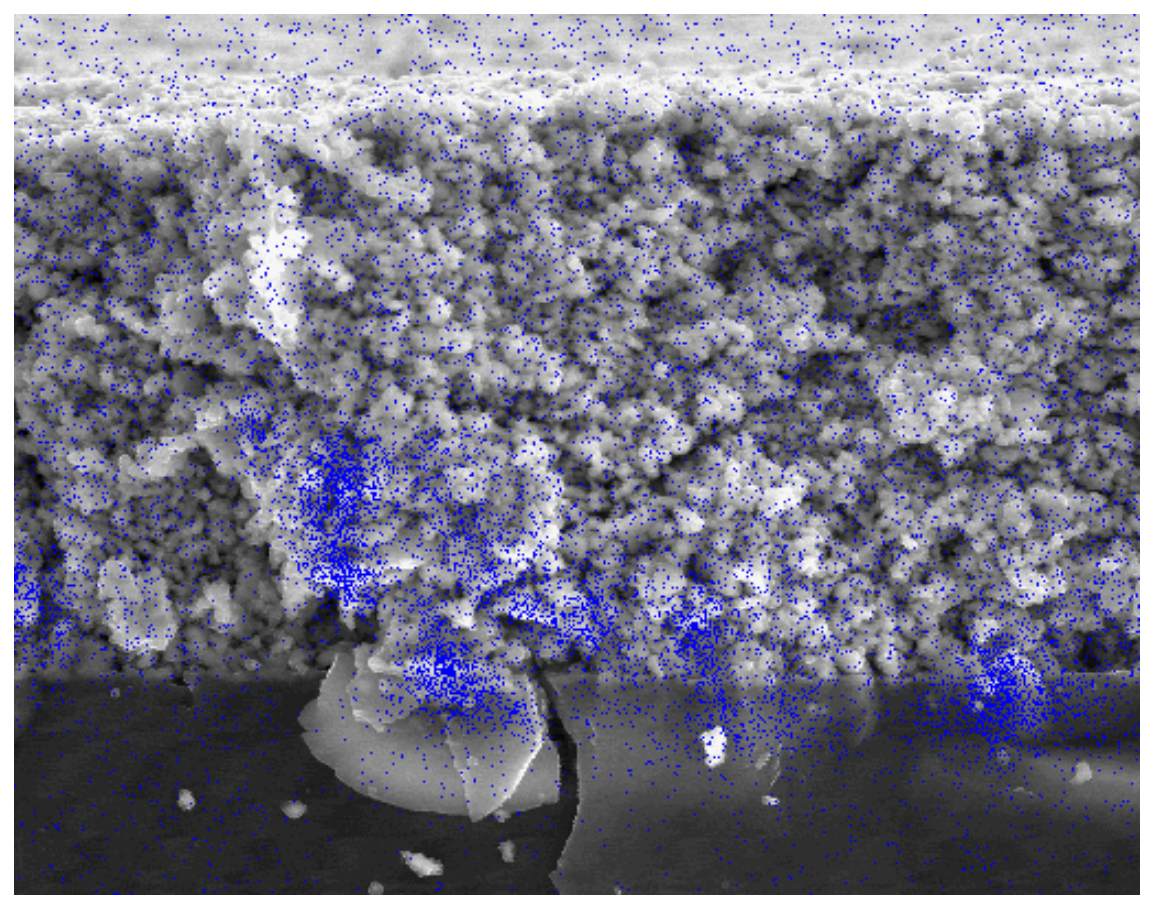

Figure 35 X-ray map of silver overlay of SEM micrograph of silver-LSM cross-section (2-weeks exposure)

In the above overlay, no reason for the higher rates of silver diffusion into the LSM in the region to the left of center is visible. It is possible that a localized area of high porosity is 
present, facilitating greater bulk diffusion in that area. It is apparent though that after only two weeks of exposure large quantities of silver has diffused nearly halfway through the LSM.

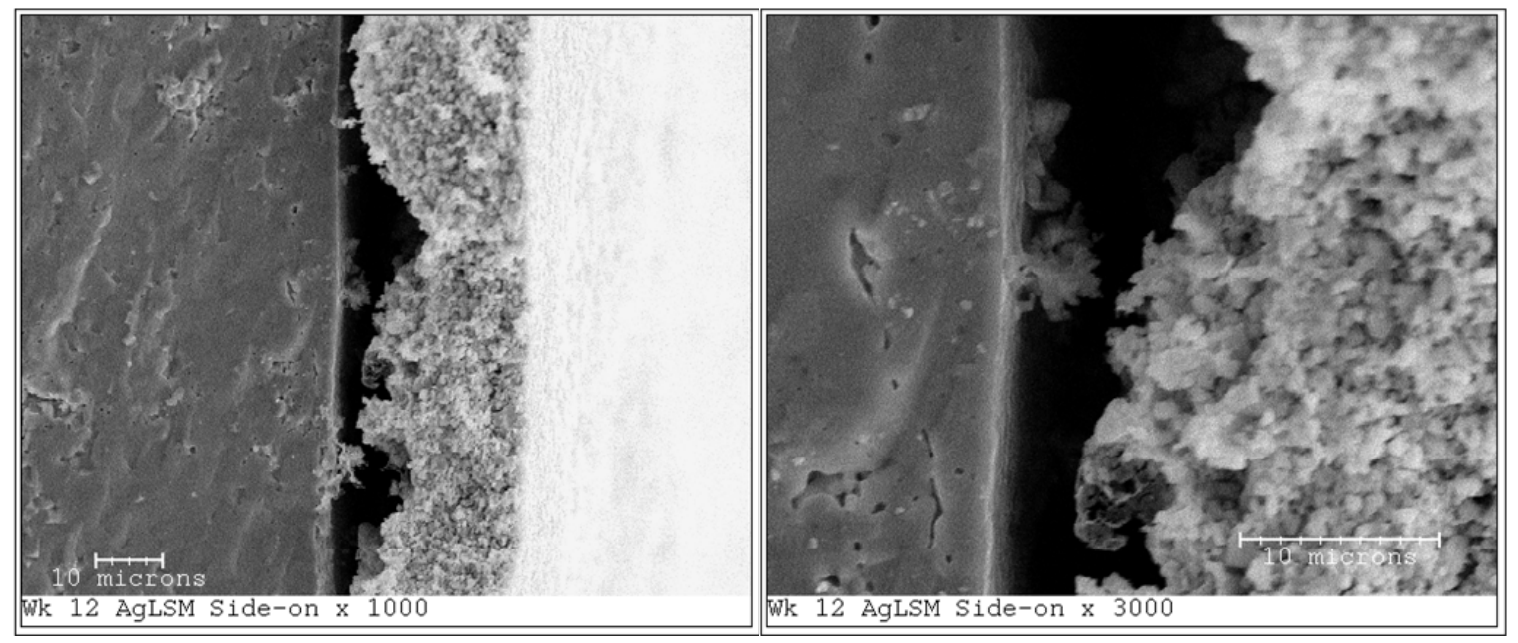

Figure 36 SEM micrograph of silver-LSM cross section at $1 \mathrm{k}$ and $3 \mathrm{k}$ magnification (12-weeks exposure)

In the above cross section of the silver-LSM sample, taken after 12 weeks exposure the two images show the sample at the same location but at different magnifications. In both images, the YSZ substrate can be seen at left, while the LSM coating layer is visible at right. In the left image, the free surface of the LSM is visible. The void at the center of the sample, between the LSM and substrate, shows the location where silver was originally deposited. It is evident that bulk diffusion of the silver has occurred.

Fracture analysis at other locations within the sample indicated that all silver had diffused into the LSM coating layer. A further exhibit of the structure of the sample is presented below, taken after 14 weeks of exposure 


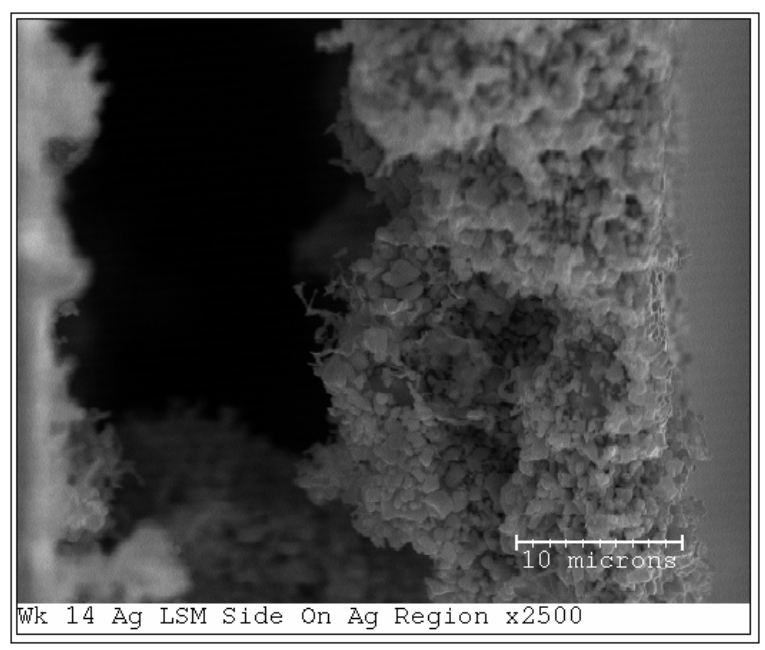

Figure 37 SEM micrograph of silver-LSM cross section (14 weeks exposure)

Analysis of the pure screen silver sample indicated that the evaporation of a circular print pattern as used in all samples occurs radially. As such, it would be expected that if silver were to be found in the original deposited location between the YSZ and LSM, it would be at the center of the sample. In light of this, a sample was broken at the center, and the surface analyzed. However, it can be seen that even at the center of the sample, all silver had diffused into the LSM. 


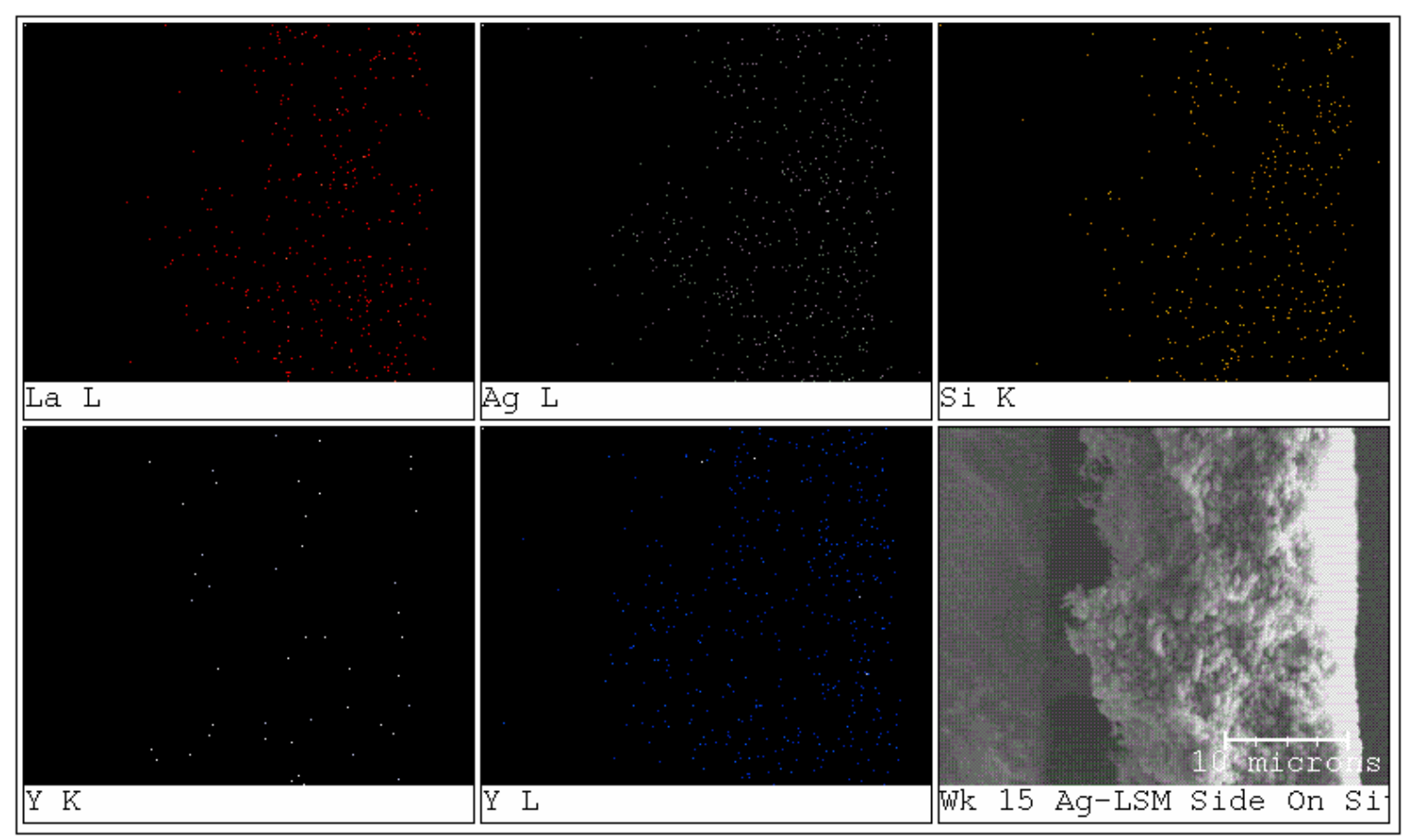

Figure $38 \mathrm{X}$-ray mapping of silver-LSM cross section documenting silver diffusion after 15 weeks exposure

In Figure 38, the bulk diffusion of silver through the LSM toward the free surface, at right, of the sample is evident. In contrast to the X-ray map taken after two weeks exposure, silver has diffused all the way to the free surface of the LSM. The greatest concentration of silver detected in the X-ray map is closer to the free surface of the LSM than to the original location of the silver.

Further analysis of the fracture surface at another location in the center of the sample revealed the same results. 


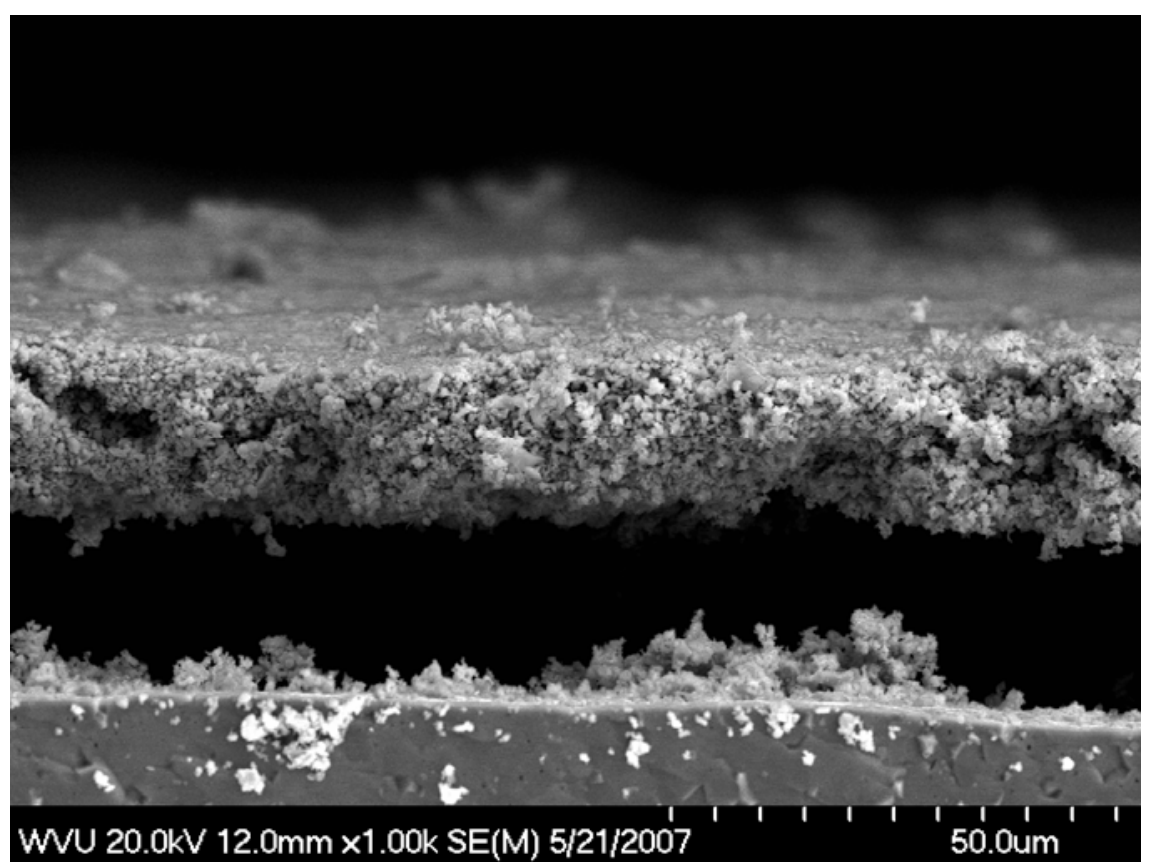

Figure 39 SEM micrograph at the center of the cross section of the silver-LSM sample (16 weeks exposure)

It is evident from the above fracture surface image that the silver layer has either evaporated or diffused completely into the LSM coating layer and that at very least all silver has diffused from the original deposition layer into the LSM. 


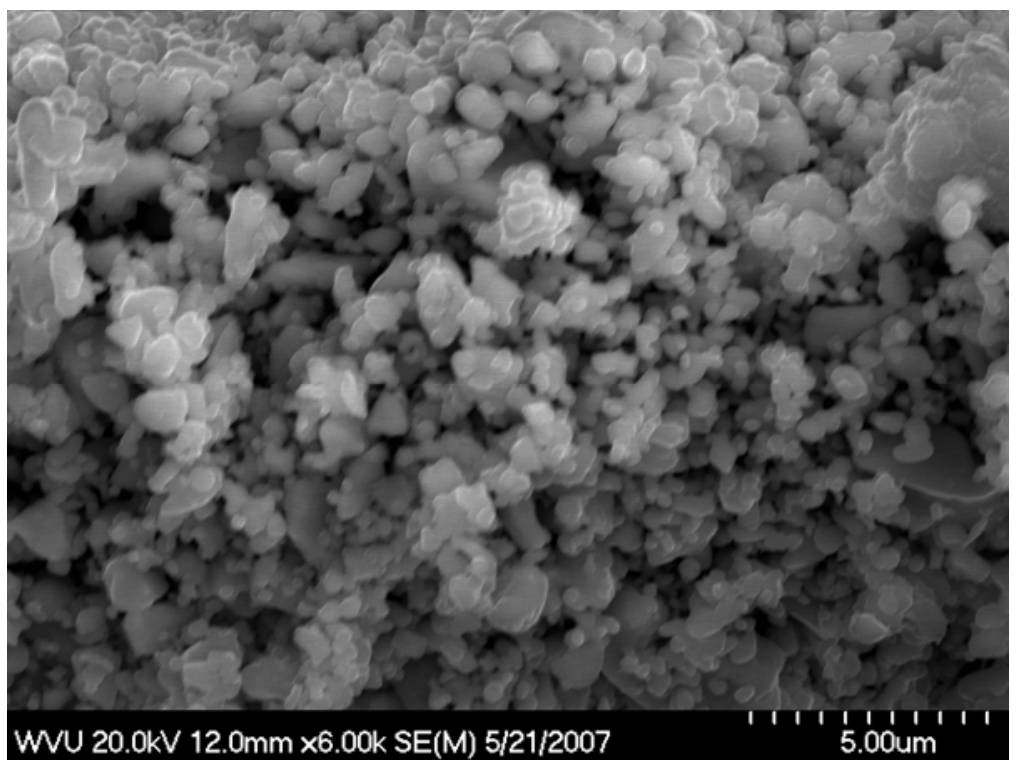

Figure 40 SEM micrograph of the LSM cross section of the silver-LSM sample (16 weeks exposure)

The above figure shows the LSM lay from the bottom of the layer to the free surface. The x-ray map of Figure 40 for silver is provided in Figure 41. It is very clear from the above image that LSM has not agglomerated into larger particles and that the particles are the same size as the smaller LSM particles discovered in the powder analysis. Even after four months exposure, LSM displays highly stable qualities. 


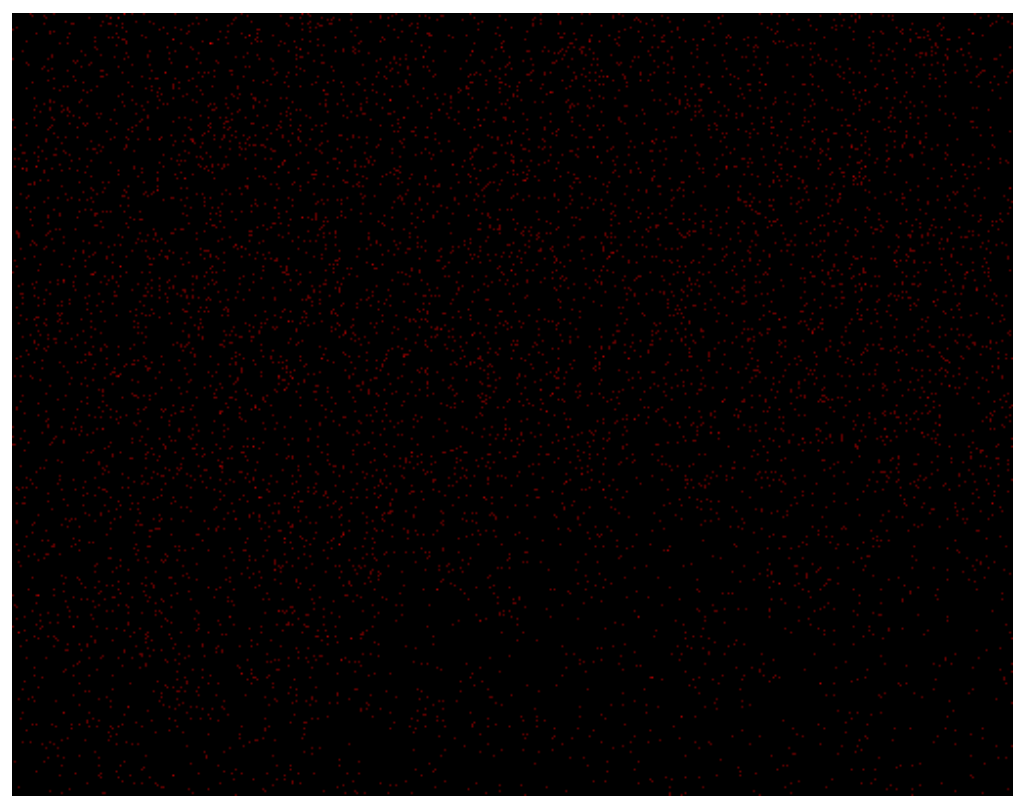

Figure 41 X-ray map for silver of LSM surface of the silver-LSM cross section (16 weeks exposure)

In Figure 40 and Figure 41, the free surface is at the top of the image. As is evident in the x-ray map, the concentration of silver increases as one moves from the bottom of the sample, which would be the boundary between the LSM layer and the originally deposited silver location, to the free surface, the concentration of silver increases. This confirms that silver is diffusing progressively further into the LSM coating layer as the exposure time continues. 


\subsubsection{Silver/10\%Ceria-LSM}

The surface morphology of the silver/10\%ceria-LSM sample behaved in much the same way as the surface of the silver-LSM sample. This was in large part due to the fact that both samples have a coating layer of LSM. As shown previous, LSM is exceedingly stable at high temperatures.

The pre-exposure surface of the silver/10\%ceria-LSM sample indicated that the surface of the sample is indeed composed of a singular material. In the backscatter image of the following pre-exposure micrograph, the light and dark areas visible on the surface are caused by more by variation of the surface topography than by variation in the elemental composition.

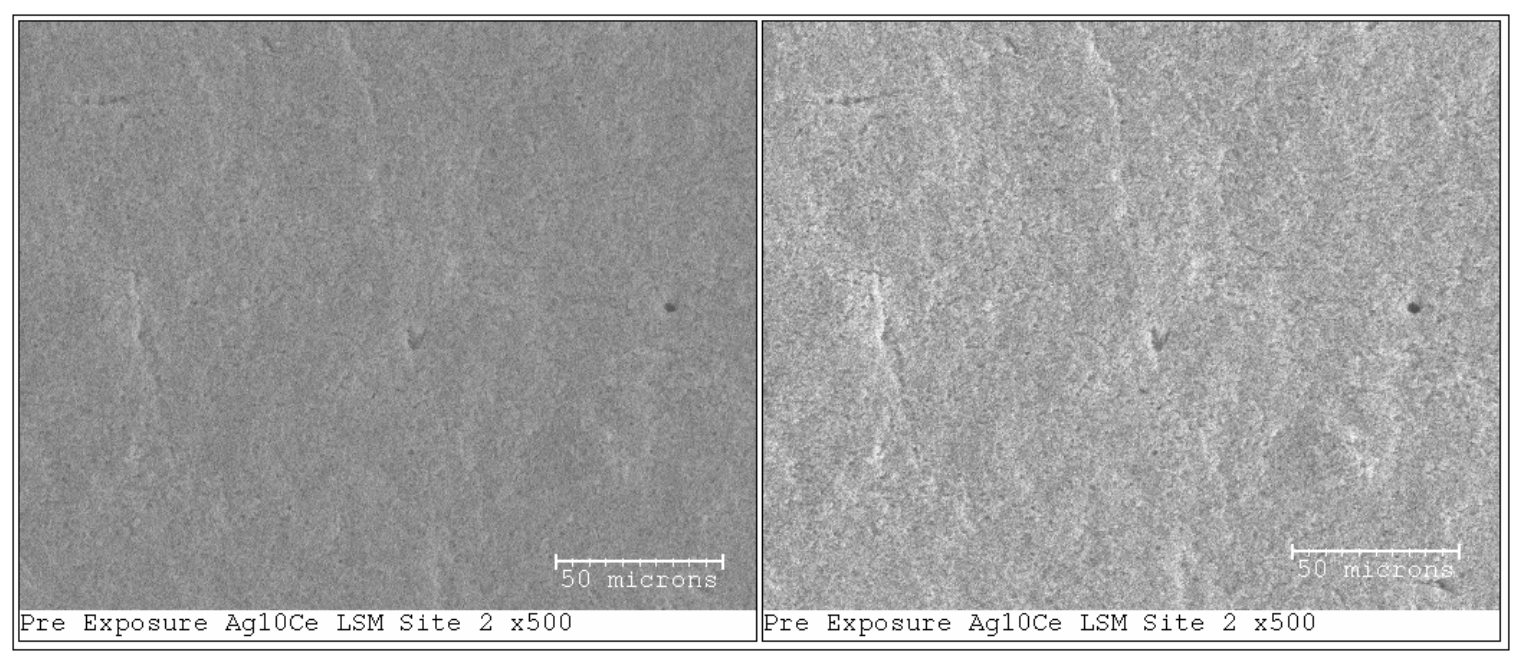

Figure 42 SEM micrograph with backscatter of pre exposure silver/10\%ceria with LSM coating

Again, X-ray spectroscopy confirmed that the surface of the sample consists of LSM. Of interest is the fact that in the image above, the imprints of the screen used in the deposition process are still visible as long lines on the surface. 
Long term exposure of the silver $/ 10 \%$ ceria samples revealed little variation in the surface morphology of the sample. After four weeks exposure, thermal expansion cracks opened up in the surface of the sample, as shown in Figure 43

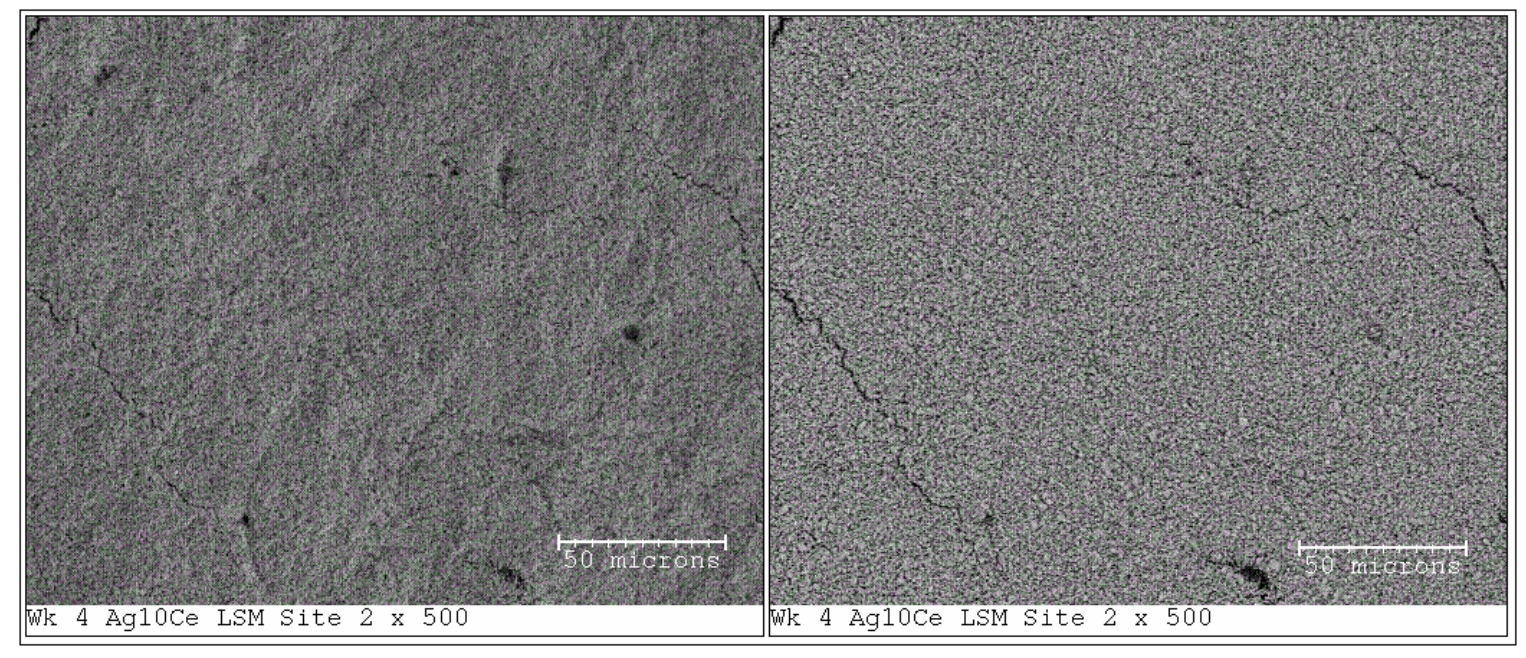

Figure 43 Crack analysis SEM micrograph with backscatter of silver/10\%ceria-LSM sample (4 weeks exposure)

The cracking was determined to be thermal expansion cracking due to the tendency of composite materials or coating layers to crack at approximately $120^{\circ}$ from each other when thermal expansion is the driving force behind the cracking. There is a slight difference in the coefficient of thermal expansion of silver in comparison to the other materials, with the CTE of silver being around $20 \times 10^{-6}{ }^{\circ} \mathrm{C}^{-1}$, while the CTE of the LSM is approximately $12 \times 10^{-6}{ }^{\circ} \mathrm{C}^{-1}$. This CTE mismatch is responsible for some cracking of the LSM layer in each of the sample types; however cracking was likely minimized by the ductility of silver ${ }^{15}$. The cracking was monitored each time SEM analysis was done in an effort to determine whether the crack would grow either in width or in length. 


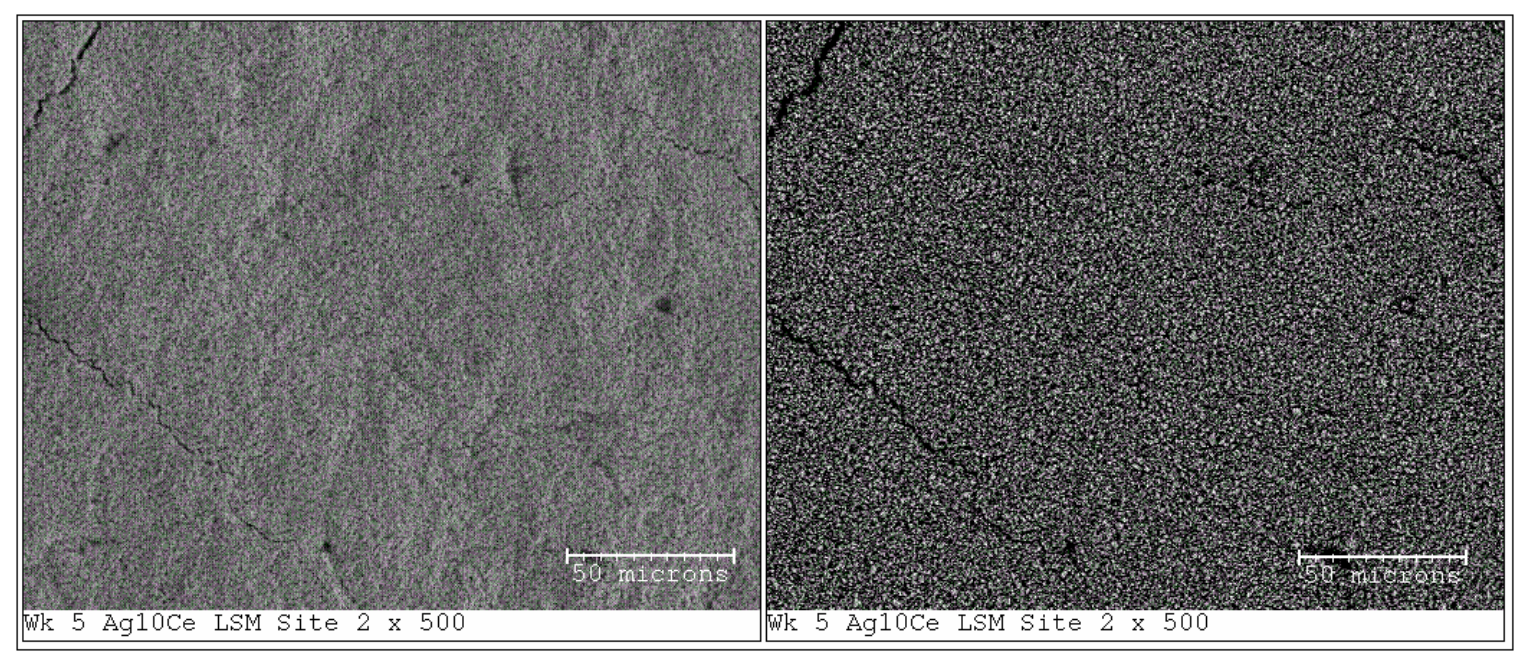

Figure 44 Crack analysis SEM micrograph with backscatter of silver/10\%ceria-LSM sample (5 weeks exposure)

As can be seen in the above image, as well as in the following micrographs, the cracking remained stable throughout the exposure time.

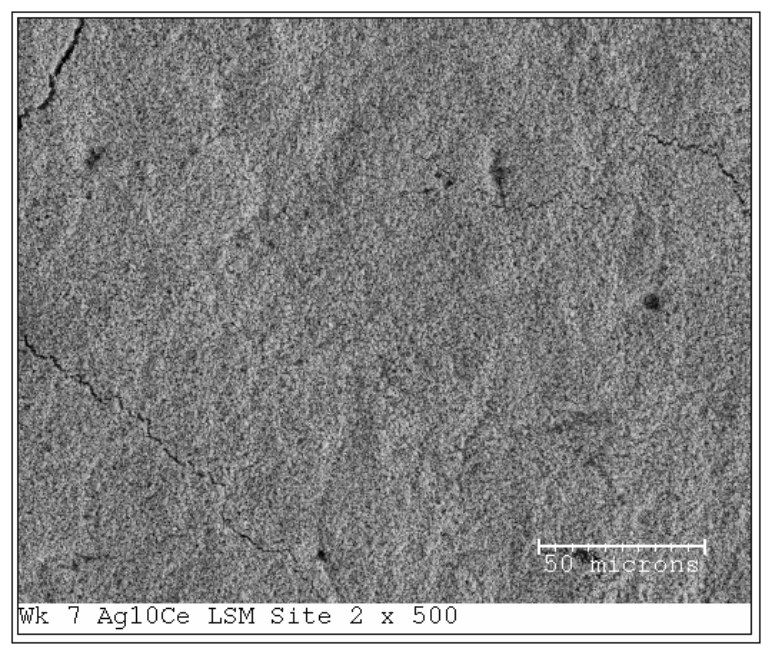

Figure 45 Crack analysis SEM micrograph of silver/10\%ceria-LSM sample (7 weeks exposure) 


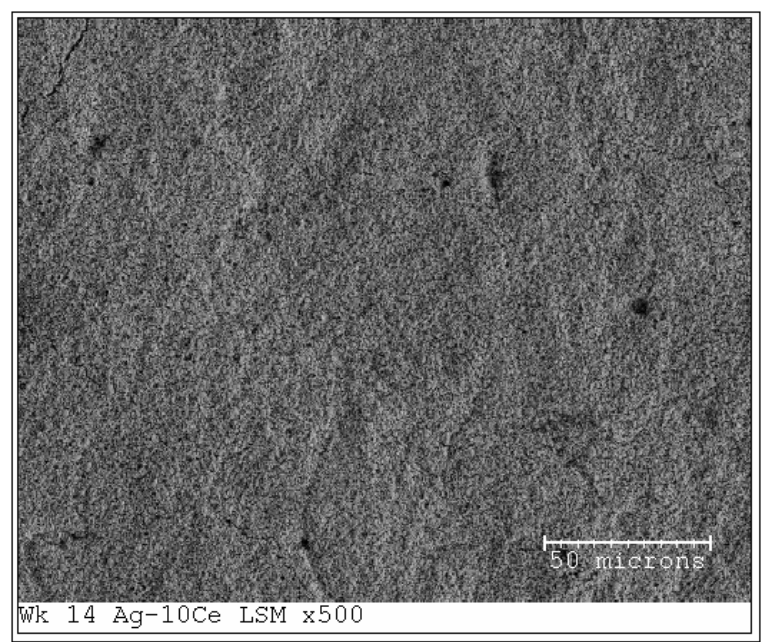

Figure 46 Crack analysis SEM micrograph of silver/10\%ceria-LSM sample (14 weeks exposure)

The high levels of evaporative stability of the LSM is shown by the lack of changes in the imprint lines that were left by the screen remain in the same general appearance that they were originally observed in the pre-exposure micrographs.

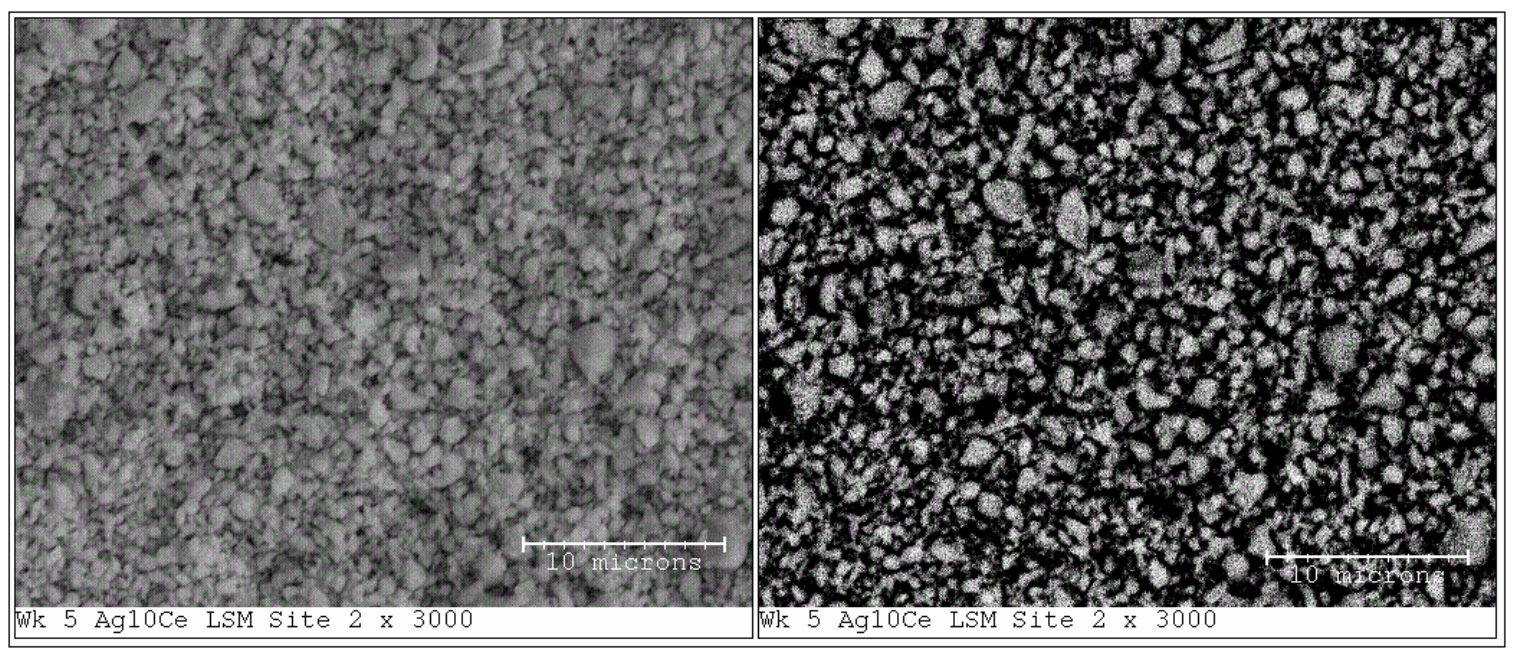

Figure 47 SEM micrograph and backscatter of silver/10\%ceria-LSM sample (5 week exposure) 


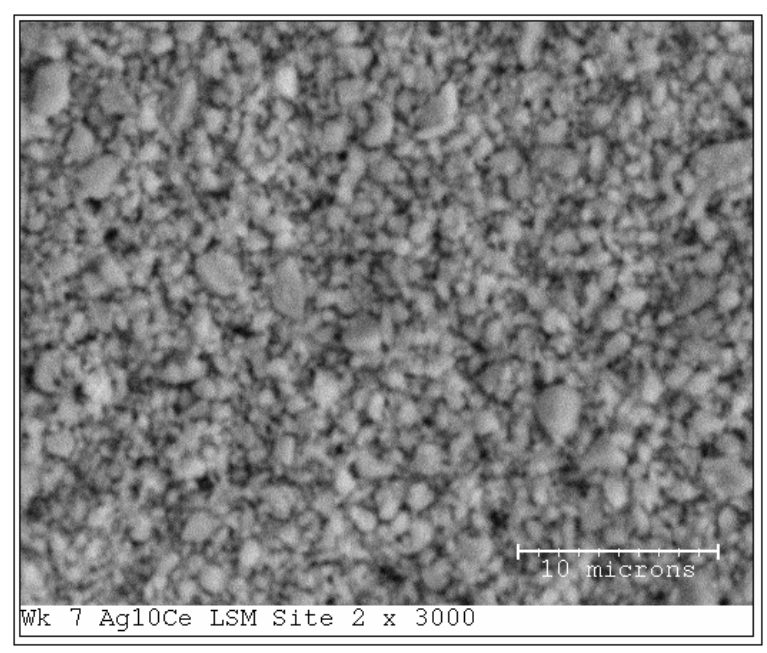

Figure 48 SEM micrograph and backscatter of silver/10\%ceria-LSM sample (7 week exposure)

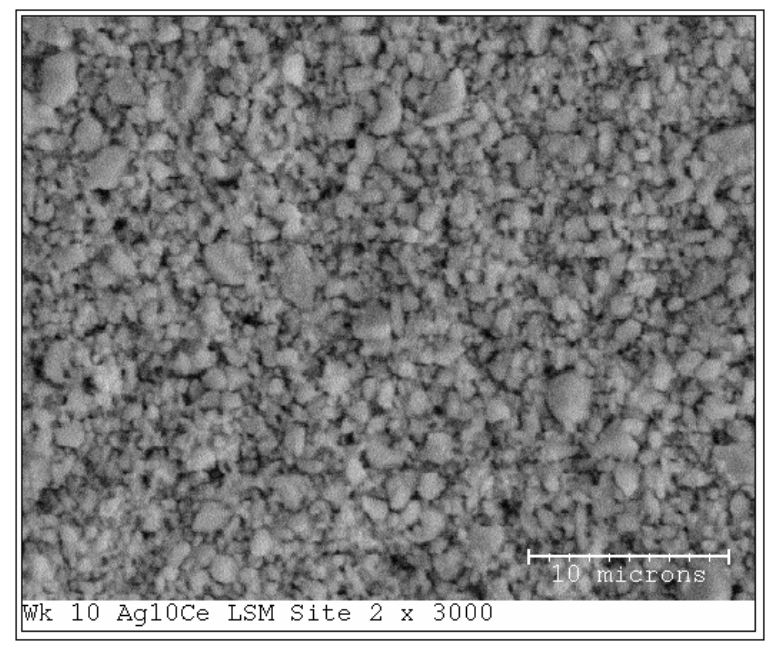

Figure 49 SEM micrograph and backscatter of silver/10\%ceria-LSM sample (10 week exposure)

After ten weeks of exposure, the surface shows no evidence that the addition of ceria as a composite layer below the LSM affects the morphology of the LSM. No coarsening of the LSM particles is visible, and the nominal size of the particles remains at $0.5-1 \mu \mathrm{m}$. After 14 weeks, there is no evidence that silver exists on the surface in a particulate form. 

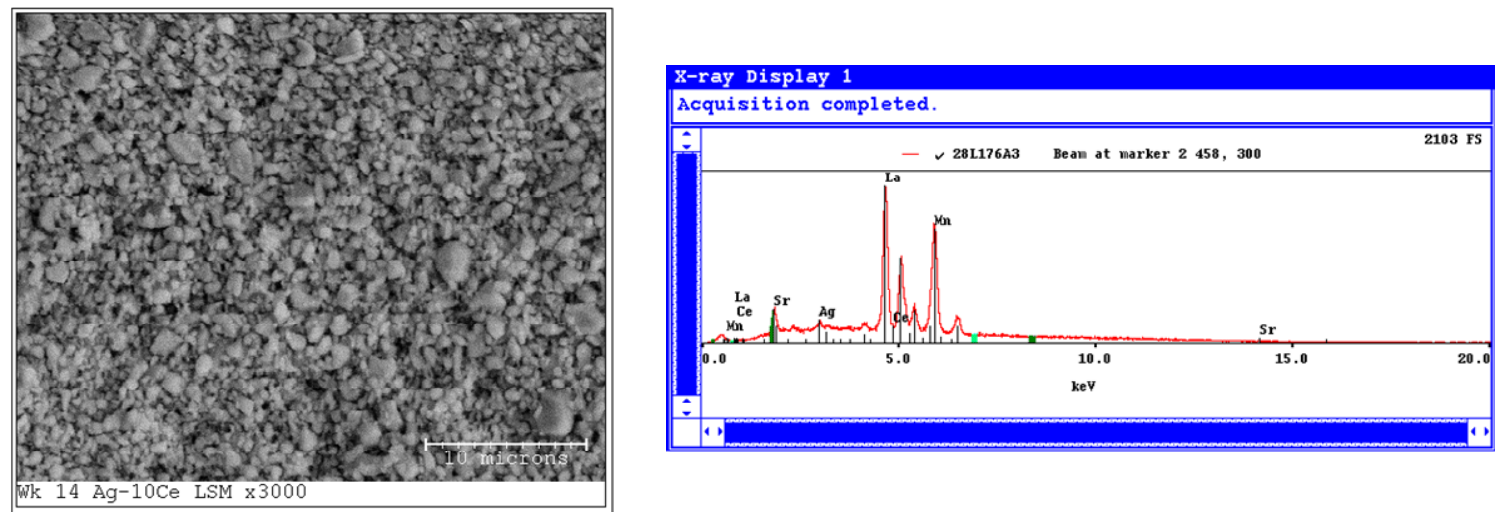

Figure 50 X-ray spectrum of entire silver/10\%ceria-LSM surface

The X-ray spectrum of the surface detects significant quantities of silver below the surface of the sample. However, no silver is visible on the surface. It seems that silver is diffusing through the LSM, but not in a particulate form.

As the samples used in this study were coated with LSM, it was important to determine whether cracks observed on the surface of the sample increase in any dimension. In order to document this, a site was chosen with both a long crack coming across it. Significant surface texture was also apparent at the location chosen.

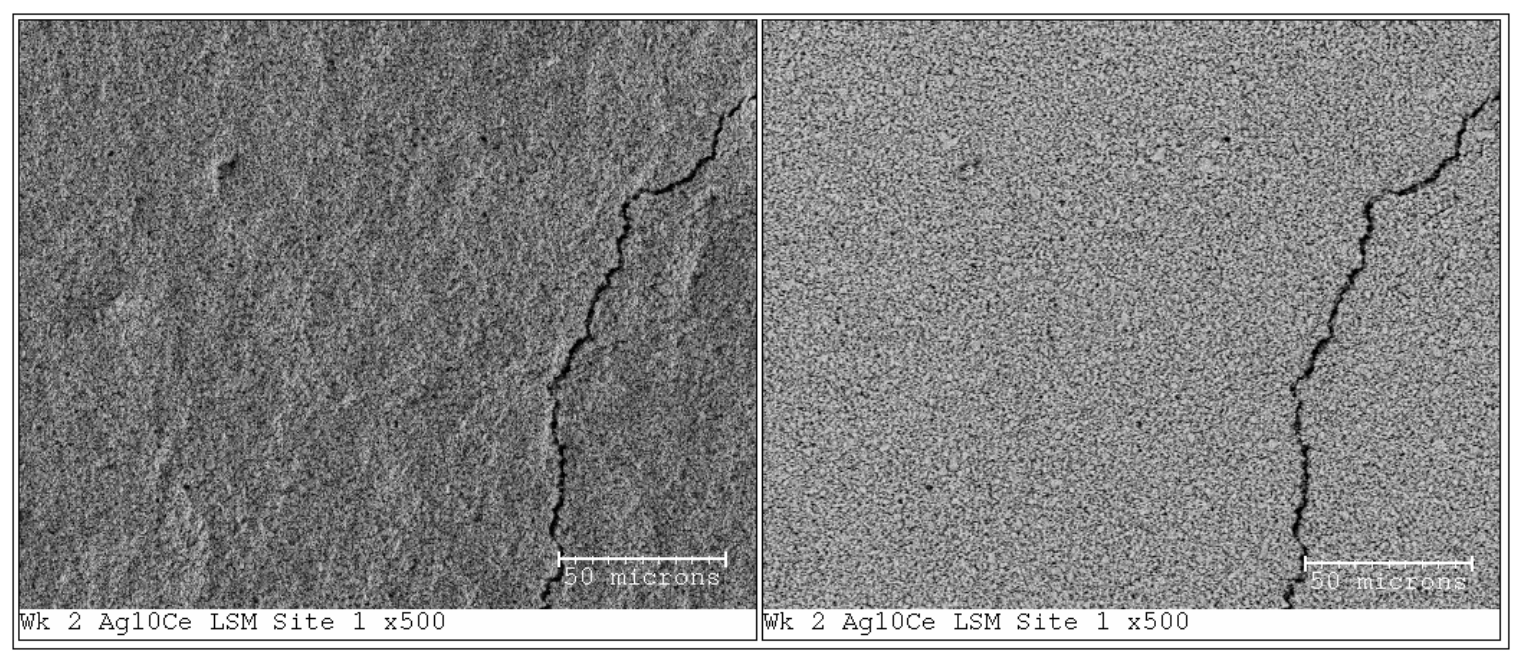

Figure 51 SEM micrograph of silver/10\%ceria-LSM surface showing cracking 
This crack opened up after the second week of exposure. Also, the cracking was substantial in the general region of the image location. As can be seen in the back scatter imaging, there is no evidence of silver particles or silver vapor coming up through the crack and depositing on either the edges of the crack, or on the surface of the LSM. Micrographs of the surface texture and the crack over the remainder of the exposure time are shown below.

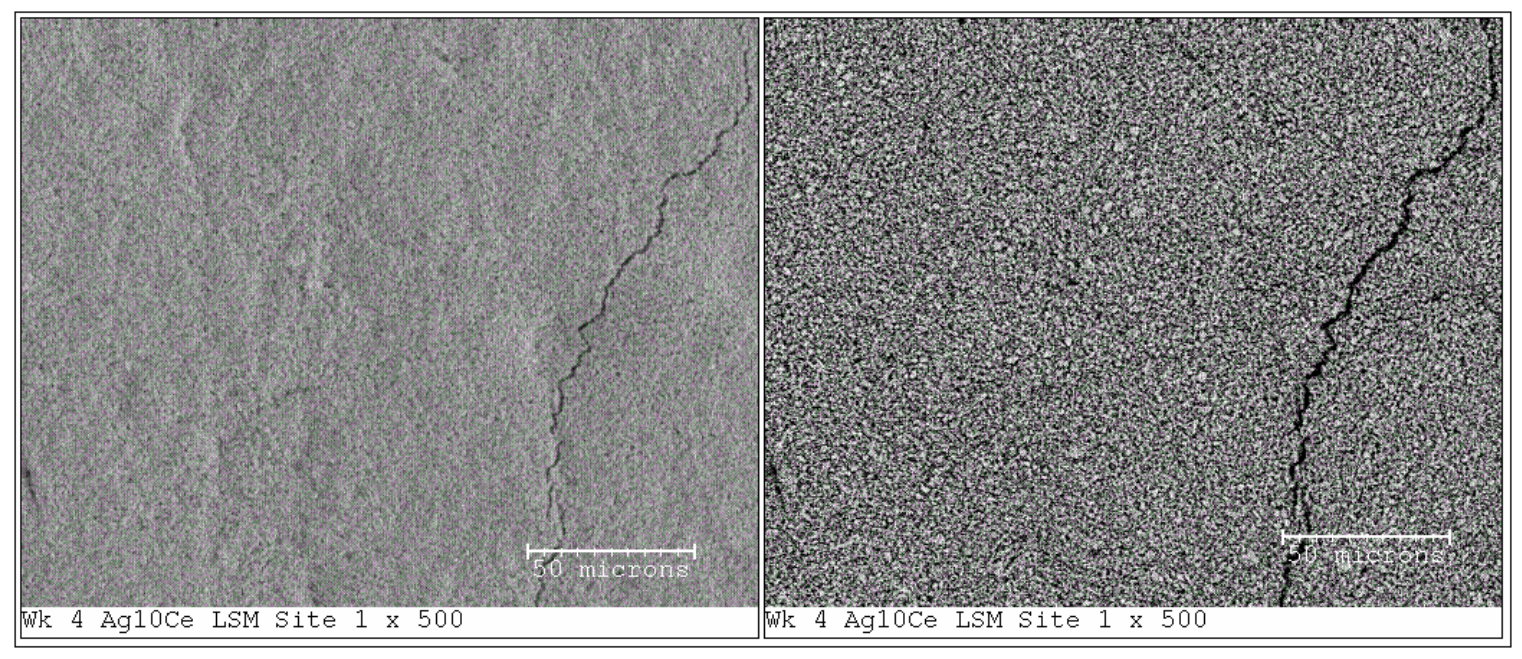

Figure 52 SEM micrograph and backscatter of silver/10\%ceria-LSM sample surface (4 weeks exposure)

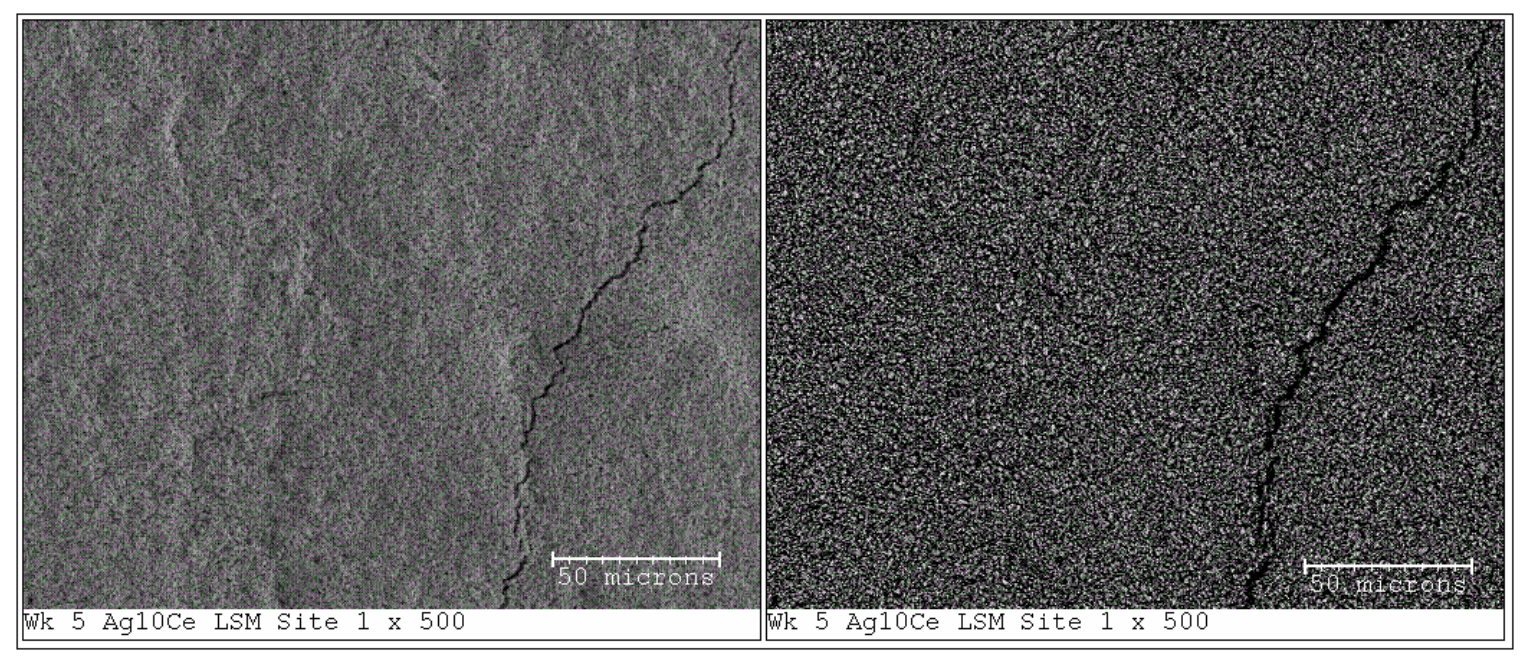

Figure 53 SEM micrograph and backscatter of silver/10\%ceria-LSM sample surface (5 weeks exposure) 


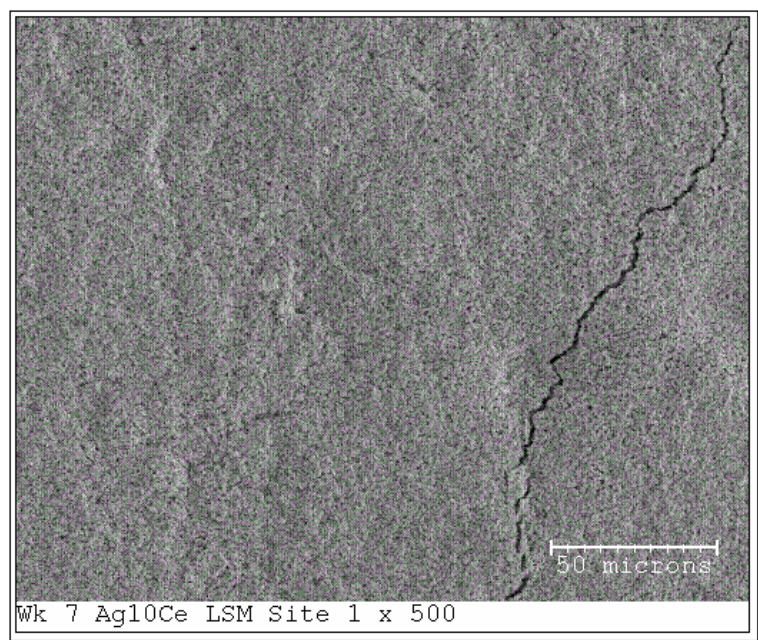

Figure 54 SEM micrograph and backscatter of silver/10\%ceria-LSM sample surface (7 weeks exposure)

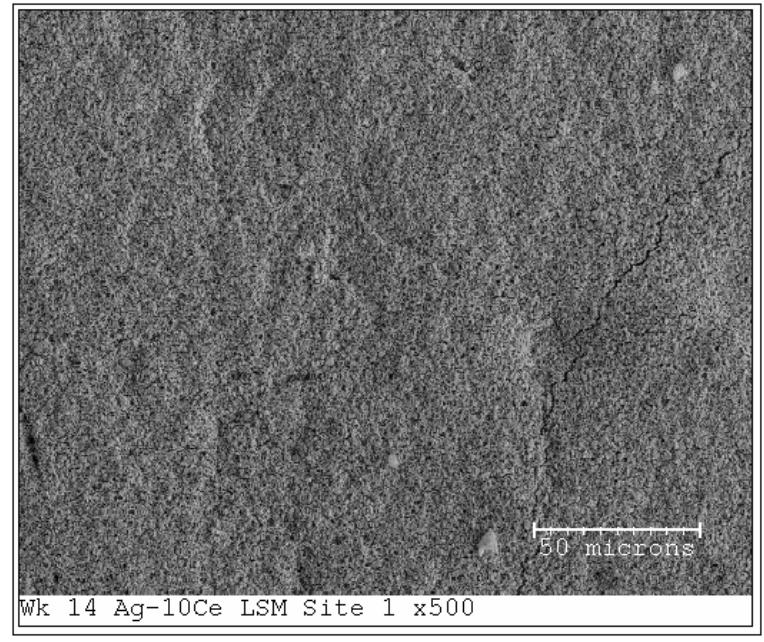

Figure 55 SEM micrograph and backscatter of silver/10\%ceria-LSM sample surface (14 weeks exposure)

The surface of the sample displayed few noticeable changes over the course of the 14 week exposure time. No expansion of the crack was noticeable, even though the sample underwent 14 complete thermal cycles during the exposure time. This provides further evidence of the thermal stability of LSM. During the exposure testing, neither silver nor ceria was ever visible on the surface of the sample. 


\subsubsection{Silver-40\%Ceria/Silver-LSM}

The silver/40\%ceria-LSM sample surface displayed similar morphology behavior to the surface of the silver-LSM sample. As with the $10 \%$ Ceria sample, this was in large part due to the fact that both samples have a coating layer of LSM.

The pre-exposure surface of the silver $/ 40 \%$ ceria-LSM sample indicated that the surface of the sample is indeed composed of a singular material.

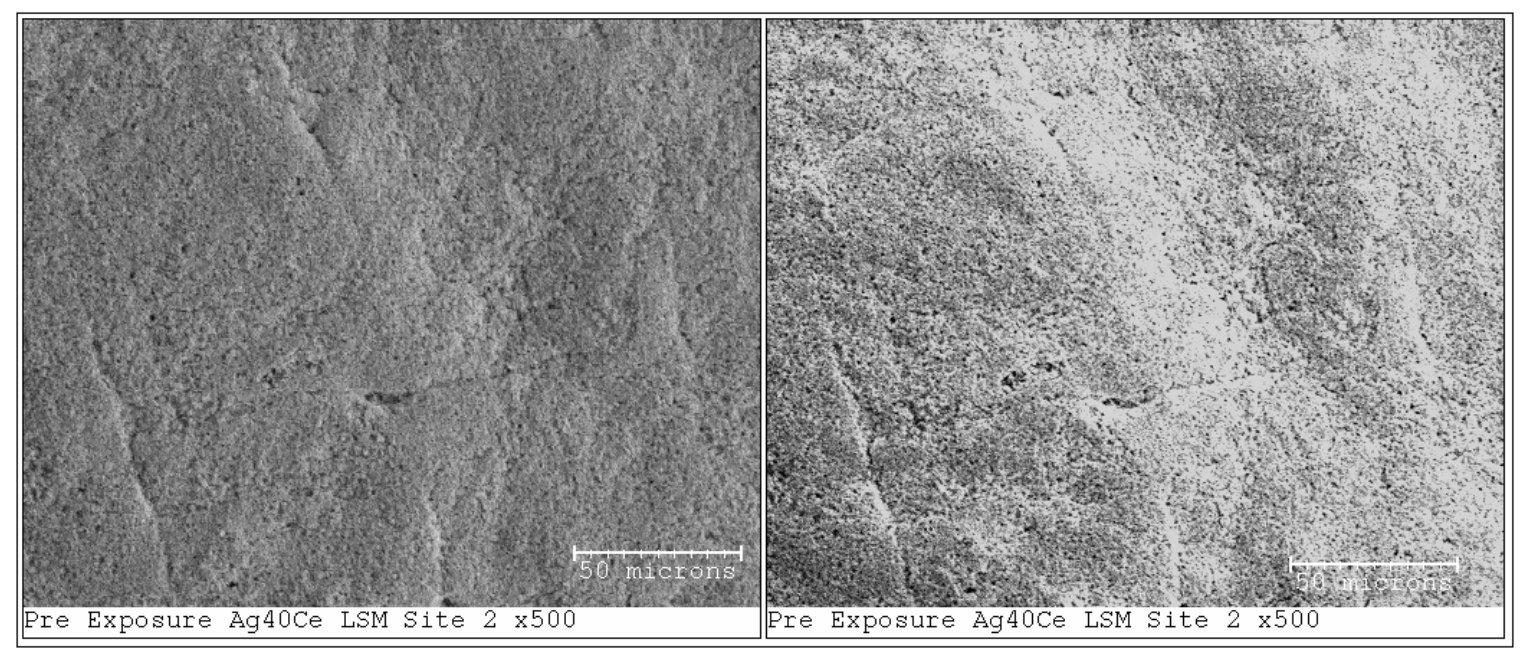

Figure 56 SEM micrograph and backscatter of pre exposure silver/40\%ceria-LSM sample surface

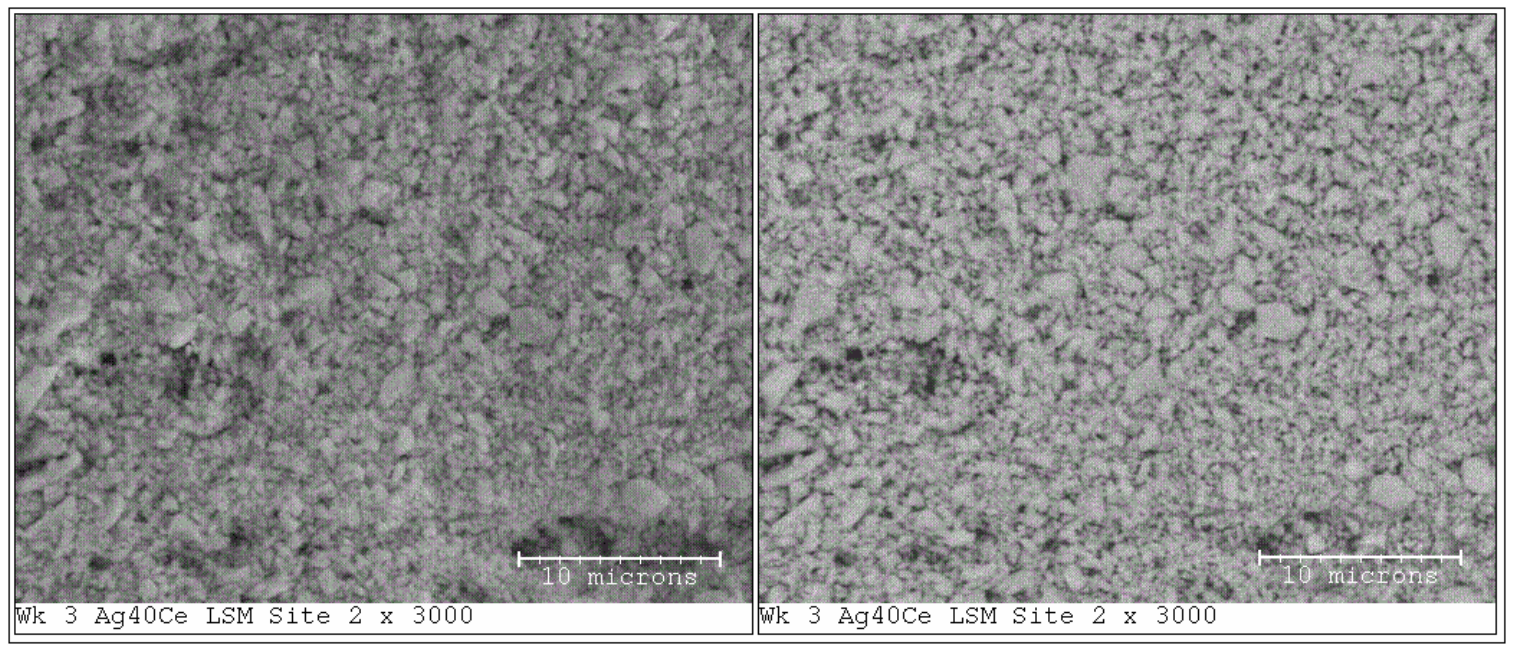

Figure 57 SEM micrograph and backscatter of silver/40\%ceria-LSM sample surface (3 weeks exposure) 
After three weeks of exposure, the backscatter image of the sample surface indicates that the surface is still composed of one material, with no silver coming through the surface.

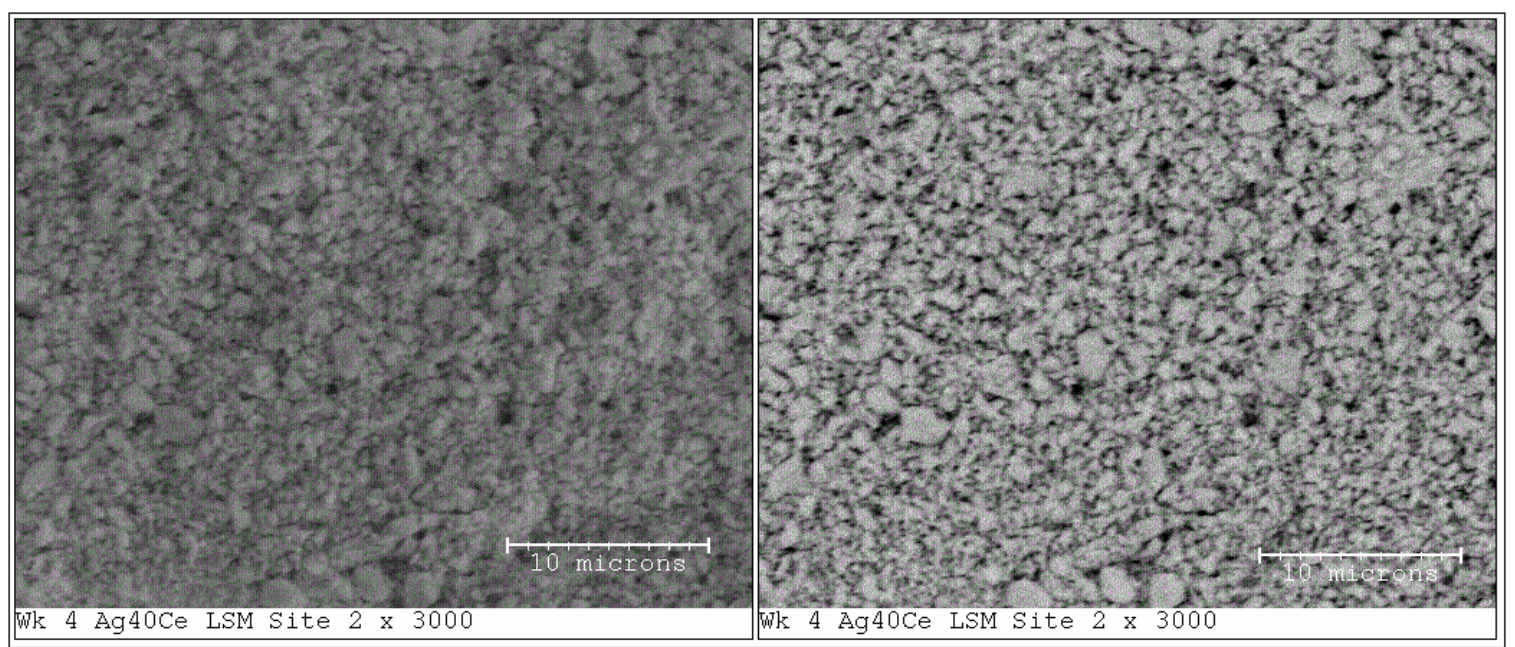

Figure 58 SEM micrograph and backscatter of silver/40\%ceria-LSM sample surface (4 weeks exposure)

After 4 weeks of exposure, the silver $/ 40 \%$ ceria sample surface micrograph and backscatter indicates no silver on the surface. This fact is important, as week four was the exposure period during which the silver $40 \%$ ceria sample experienced rapid nonlinear mass loss. This means that silver is evaporating from the surface after diffusing through the LSM layer as vapor or atomically.
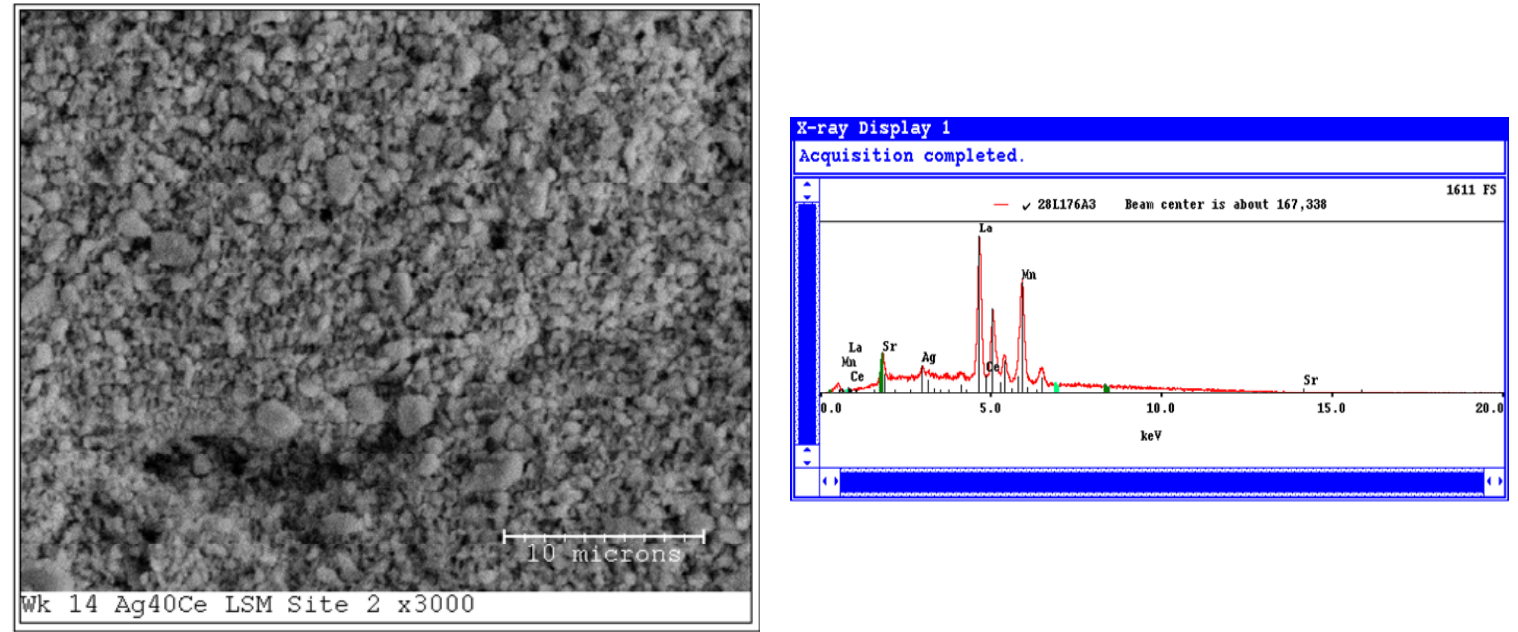

Figure 59 X-ray spectrum of silver/40\%ceria-LSM surface after 14 weeks exposure 
Throughout the exposure time, the LSM surface of the silver $/ 40 \%$ ceria sample showed no evidence of silver on the surface or LSM coarsening. The X-ray spectrum of the sample taken after 14 weeks of exposure indicates that silver remains within the sample, but at far lower detectable levels that the ceria layer beneath the LSM coating.

\subsubsection{Silver/40\% Ceria Fracture Surface Analysis}

To determine the behavior of the silver when a silver/ceria composite layer is included in the sample, the sample was broken at the center after 16 weeks of exposure. The rate of silver evaporation is believed to be lowest at the center of the sample, so it will be most detectable there. Also, the 16 week fracture surface of the silver-LSM sample was done at the center, allowing for a realistic comparison.

The center of the silver/ceria sample indicated that the silver has undergone bulk diffusion into the LSM layer and that much of it has evaporated. In the micrograph presented below, the free surface is at right.

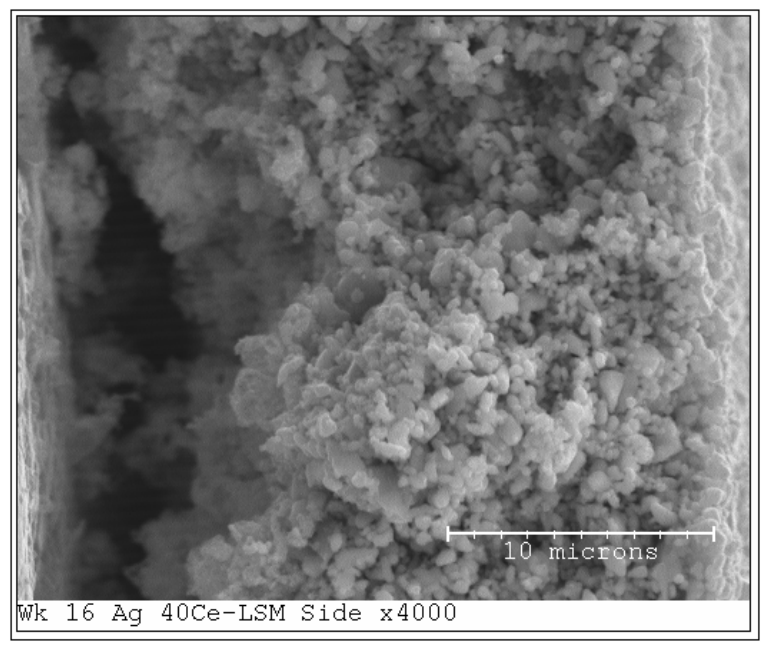

Figure 60 Cross section SEM micrograph of the silver/40\%ceria-LSM sample (16 weeks exposure) 


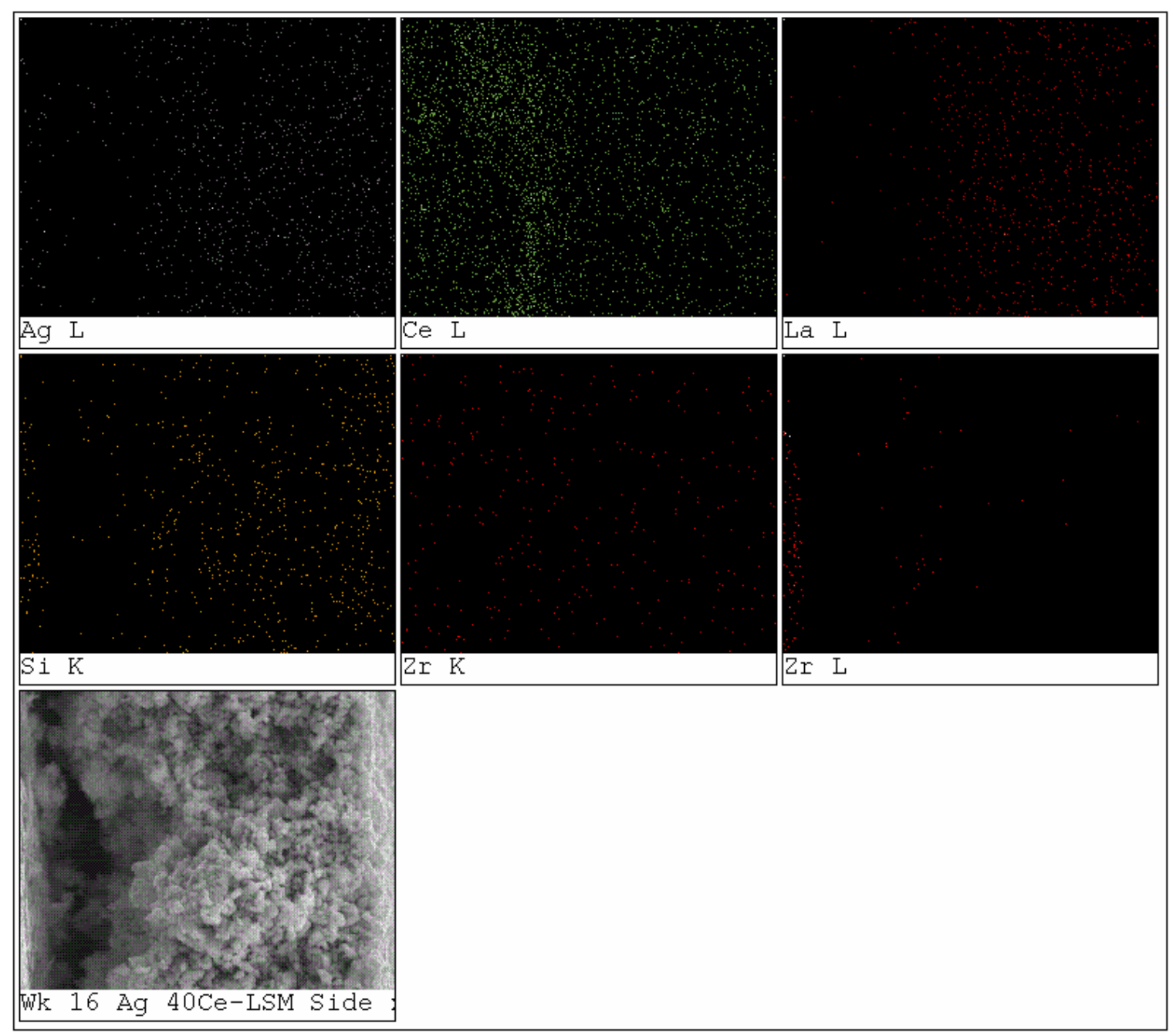

Figure 61 X-ray map of the cross section of the silver/40\%ceria-LSM sample (16 weeks exposure)

In the X-ray map of the fracture surface, ceria is shown to be stable and to have undergone no diffusion into the LSM, while silver has almost completely diffused into the coating layer. It seems that some silver has been held in place by the addition of ceria, but the greatest concentration of silver is within the LSM, with the concentration increasing as one nears the free surface. No concentrations of silver in the LSM layer sufficient to indicate particle movement is visible, meaning it is either vapor or atomic diffusion of silver that is occurring. 
To document the amount of silver that remains within the ceria layer, a piece of the LSM coating layer was removed and the bottom of it inspected. The bottom surface of the LSM coating layer shows a very rugged texture consisting almost entirely of ceria.

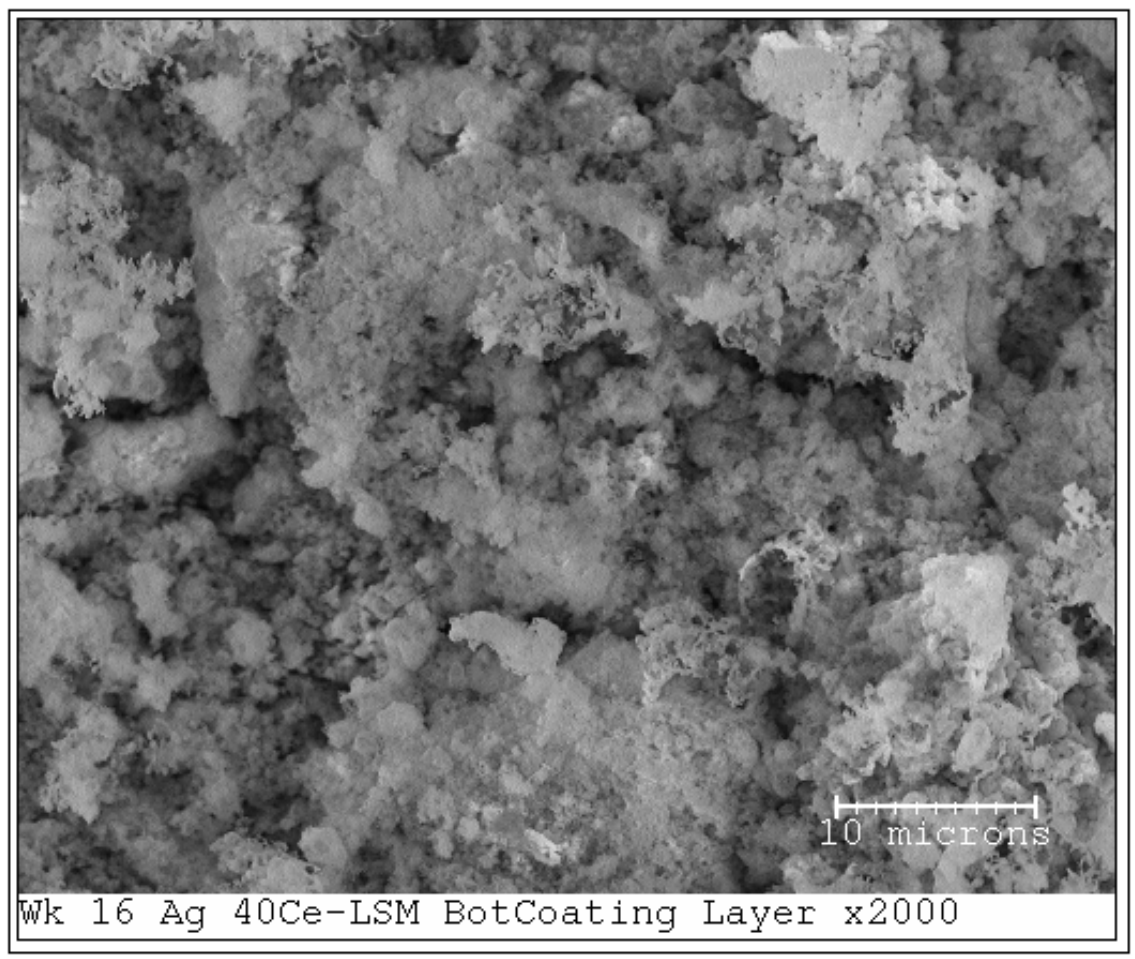

Figure 62 SEM micrograph of the bottom of the LSM layer (16 weeks exposure)

The ceria found on the bottom of the LSM layer has very similar texture to the ceria coating the silver particles observed in the $40 \%$ ceria powder analysis and shows no evidence of agglomeration. It appears that all silver has diffused into the LSM layer, with no silver particles visible on the bottom surface of the LSM layer. It seems that ceria is highly stable, as shown by the thin, wispy structure of the ceria.

X-ray mapping of the bottom of the LSM surface revealed that the surface is coated with ceria and that no silver particles remain below the LSM layer. 


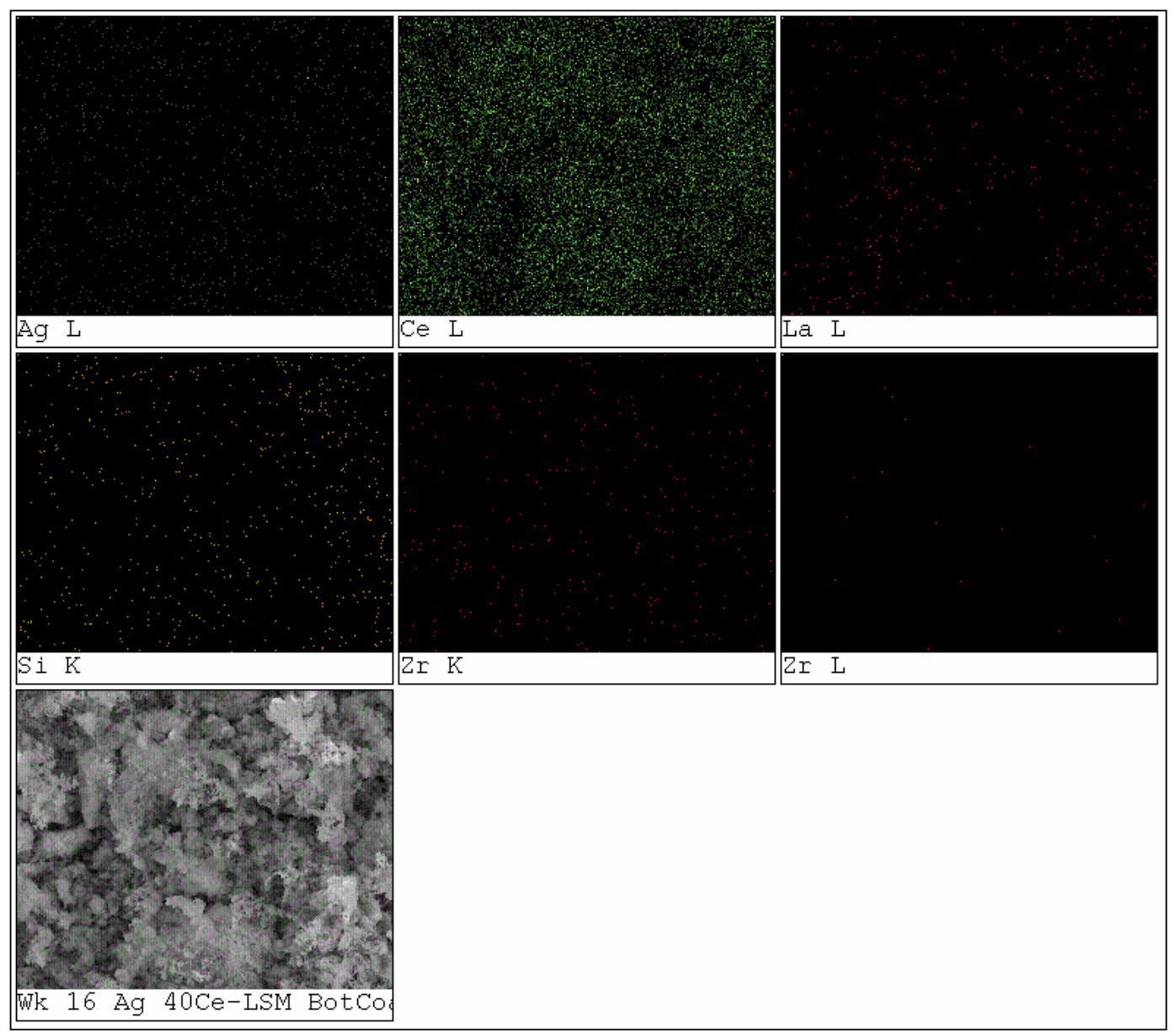

Figure 63 X-ray map of the bottom of the LSM layer of the silver/40\%ceria-LSM sample (16 weeks exposure) 


\subsubsection{Screen Printed Pure Silver}

Pure screen printed silver samples were placed in the tube furnace to observe the behavior of silver nano-particles in a SCE. These particles were analyzed in the SEM in the same fashion as the silver-LSM samples. Of note is the fact that the pure silver sample consisted of only two screen print passes. This was done to limit the amount of silver nanoparticles that were deposited in order to enhance understanding of the particles behavior by making them more visible.

The printed silver surface showed some signs of sintering after the binder burnout stage. These signs included significant necking between the silver particles. The silver particles maintained a three-dimensional configuration and did not melt or sinter into a flat layer.

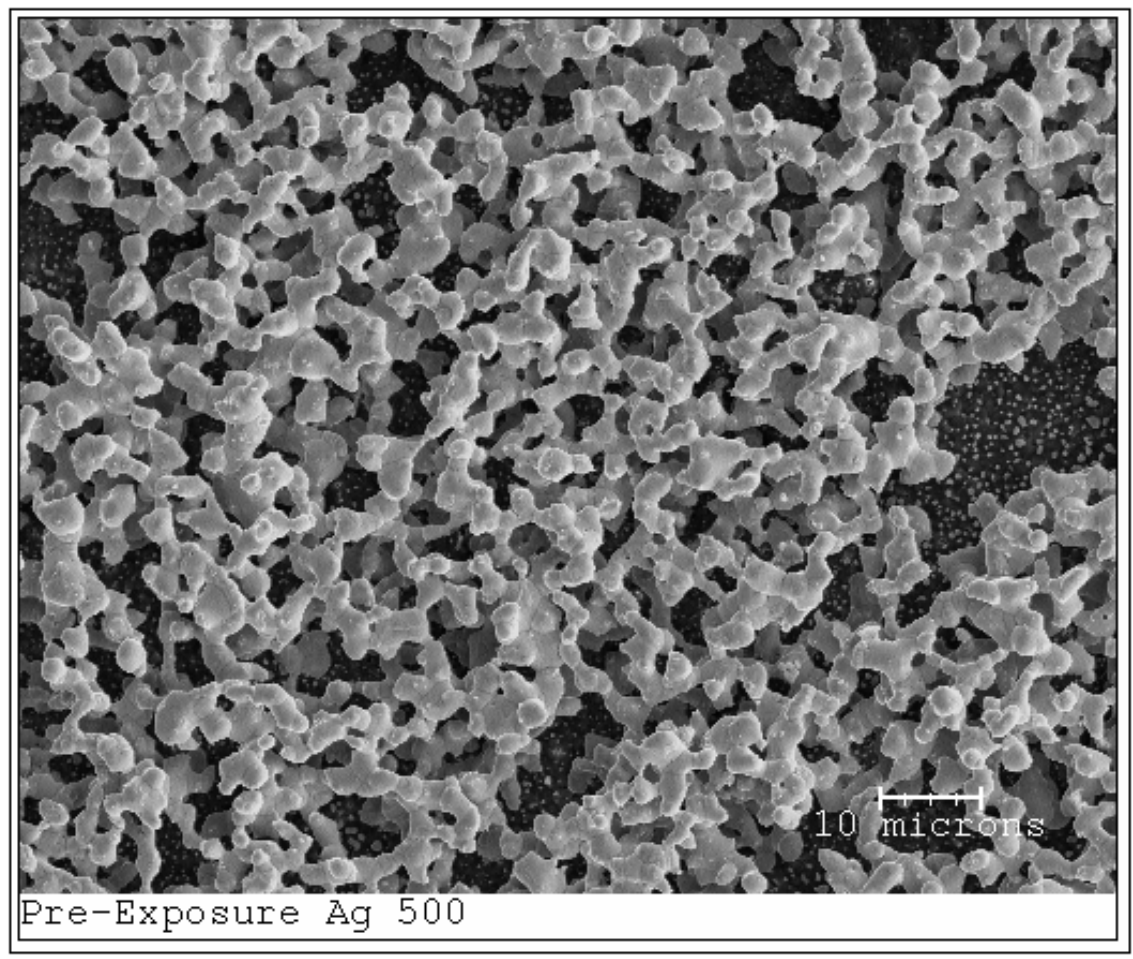

Figure 64 SEM micrograph of pre exposure printed silver 
Instead, they have a sponge-like structure with particles approximately $4-5 \mu \mathrm{m}$ in size, roughly the same size as particles in the silver powder.

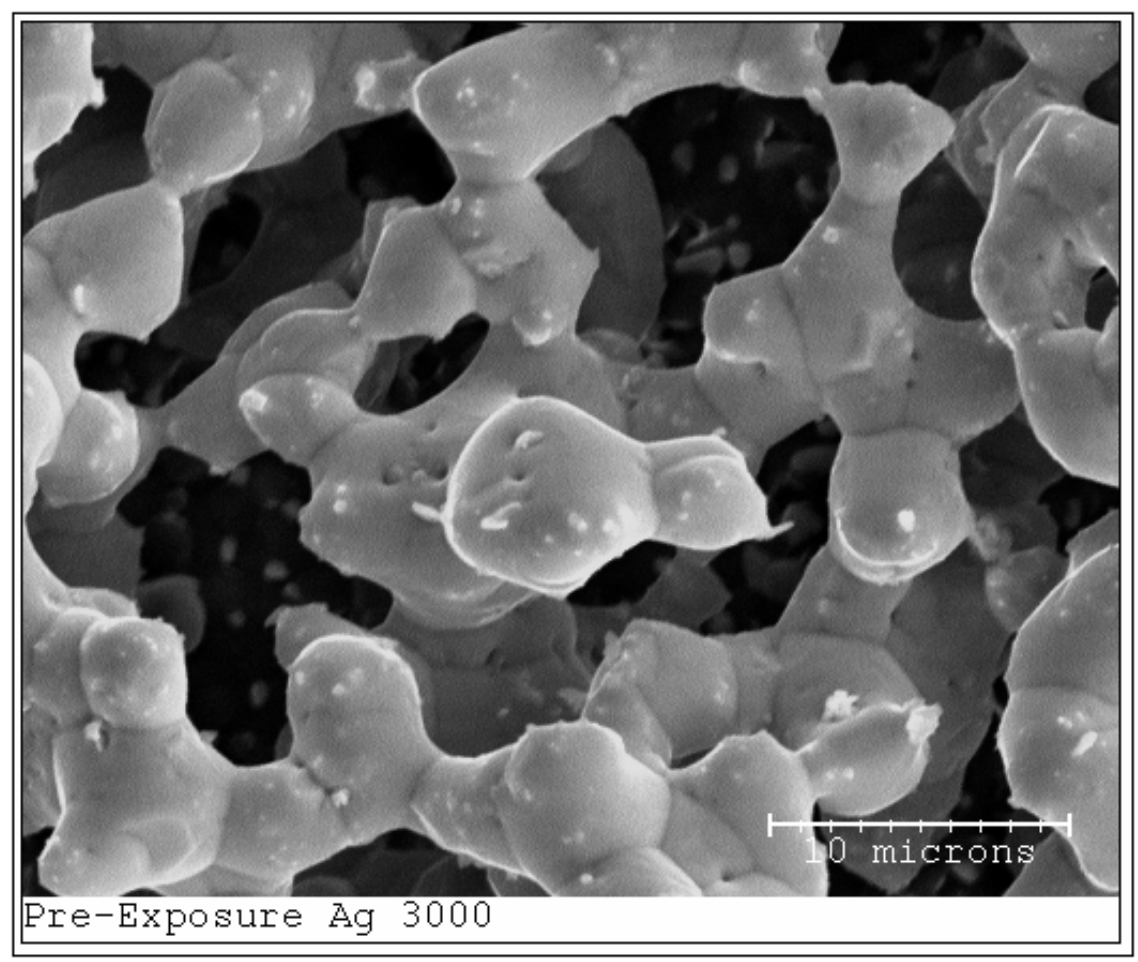

Figure 65 SEM micrograph of the pre exposure printed silver, x3k

After binder burnout the sample shows little evidence of sintering, other than significant necking between adjacent particles. In the above image, the flat dark areas are exposed sections of the YSZ substrate the sample was printed on. These gaps are believed to be left over from the wires of the screen used during the screen printing process.

After one day of exposure the printed silver shows little increase in sintering of the particles. No particle agglomeration is evident, although some minor faceting of the surface is beginning to occur. The sponge-like texture of the sample is still clearly visible. Also, no clear signs of evaporation are present. 


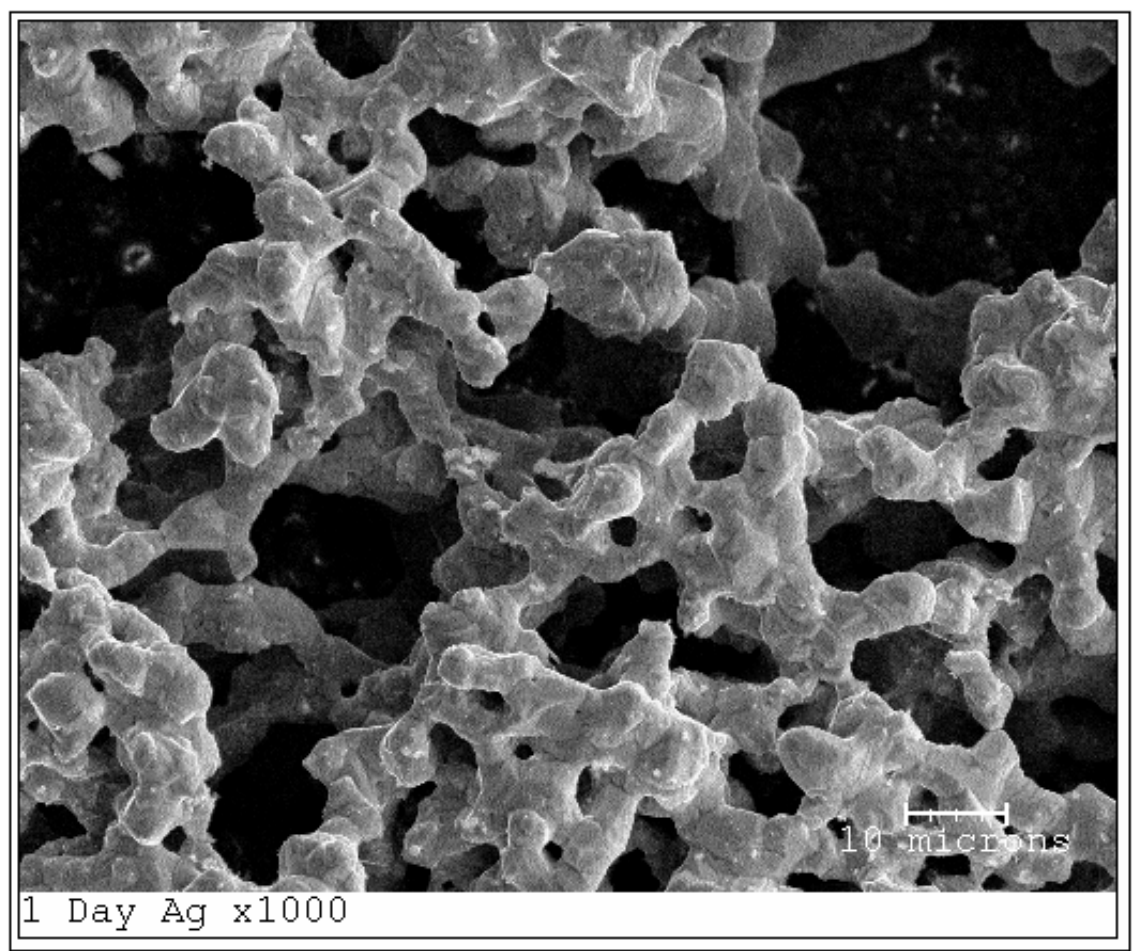

Figure 66 SEM micrograph of the printed silver after 1 day exposure

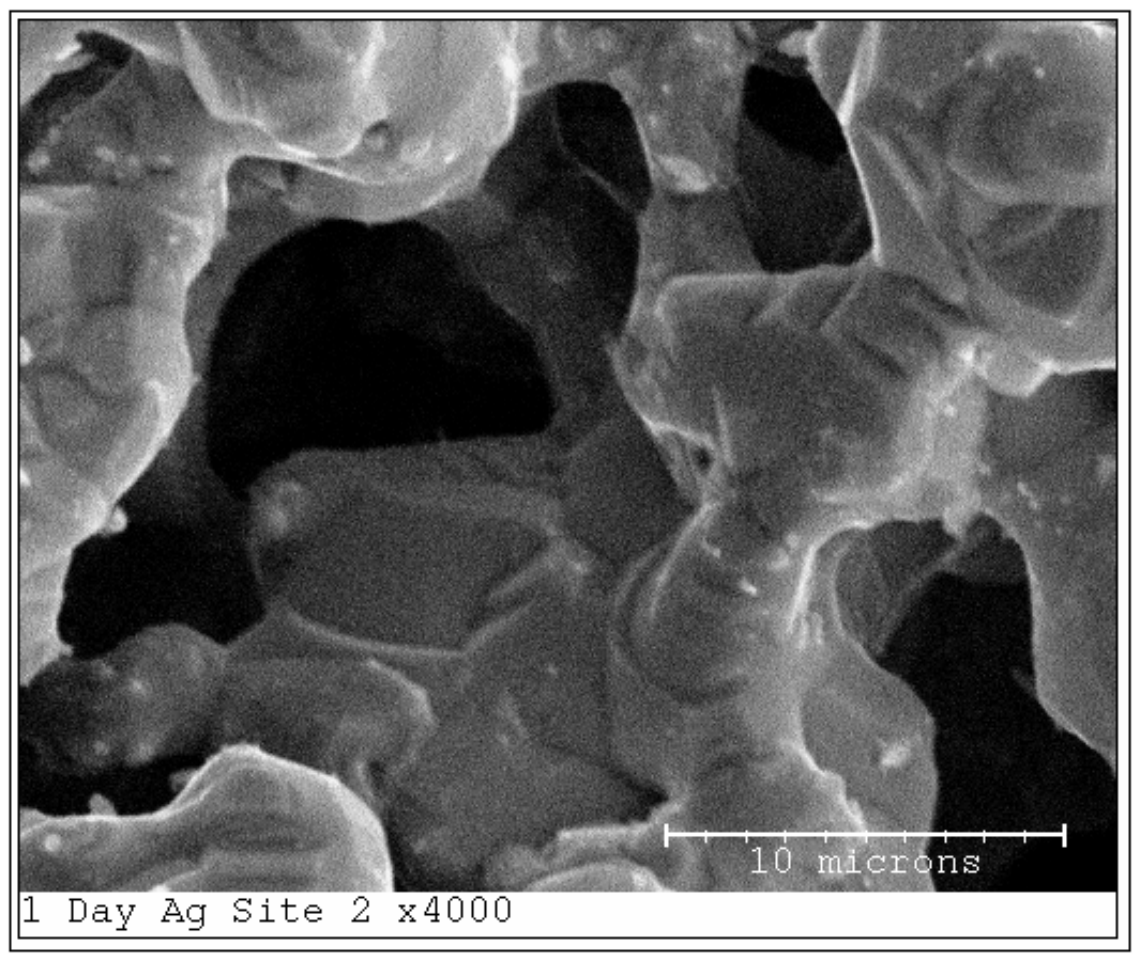

Figure 67 SEM micrograph of the printed silver after 1 day exposure showing faceting 
In the above image, it seems that most faceting is occurring in the neck regions or along grain boundaries. Since facets are caused by a rearranging of the atoms of the sample to reduce its free surface energy, it is logical for faceting to first form in the highly disordered region of the grain boundaries. This finding gave further evidence to the grain boundary degradation of silver described by Choi.

After three days of exposure, the silver displays no evidence of further sintering, or of significant evaporation. Faceting of the sample has continued to develop.

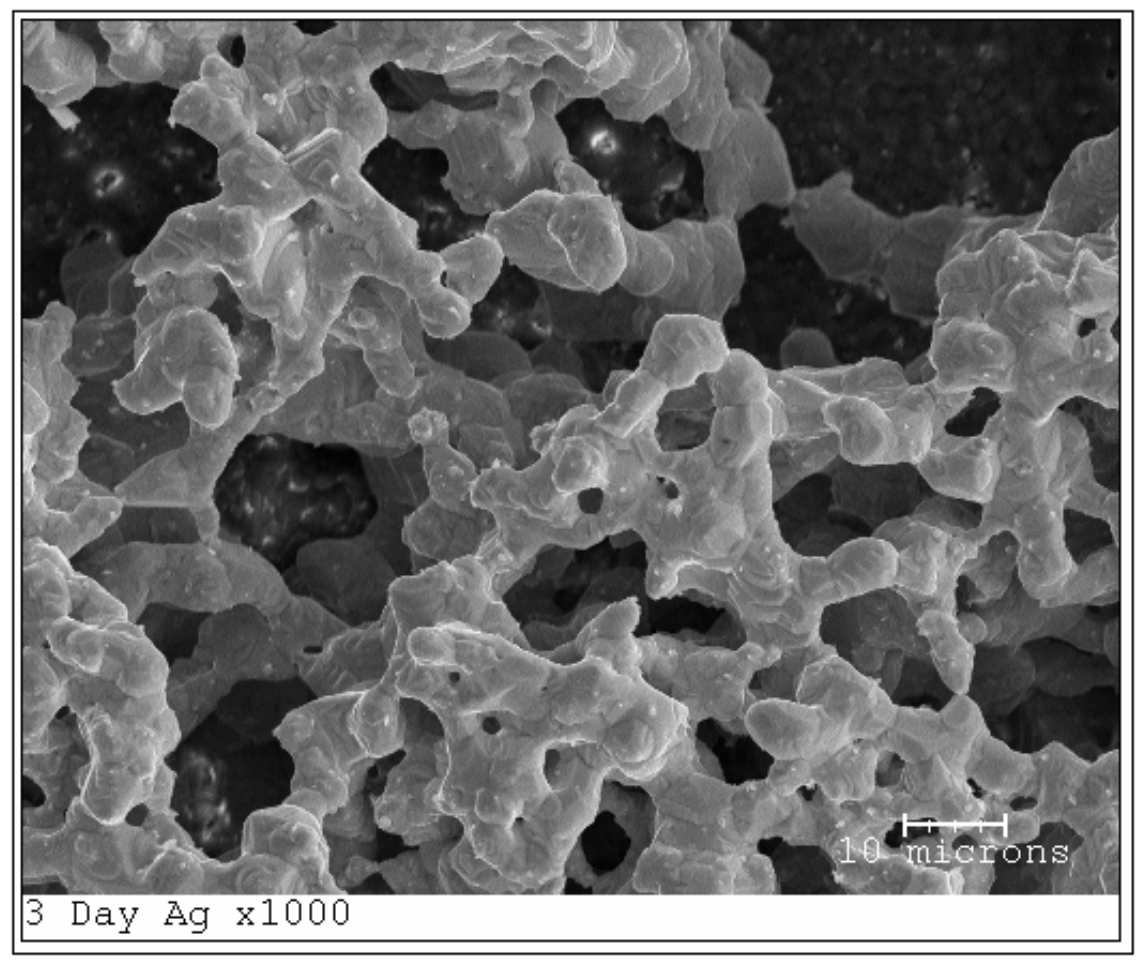

Figure 68 SEM micrograph of the printed silver after 3 day exposure showing continuing faceting

A comparison of the surface between day one and three reveals that an increase in faceting has occurred. Faceting has expanded beyond the grain boundaries to include the exposed surfaces each particle. 

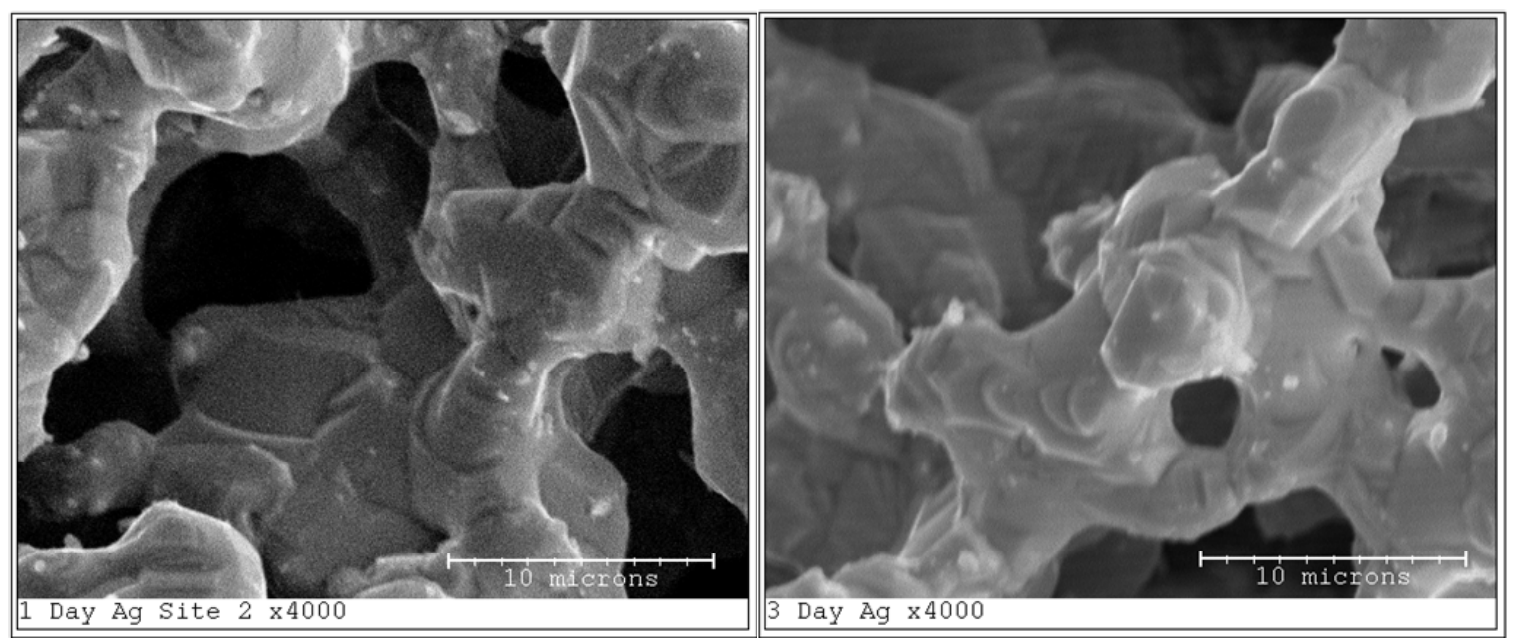

Figure 69 Comparison of faceting of silver between samples of 1 day and 3 day exposure

The surfaces of individual particles after three days are far more angular than in the one day sample. In the one day image, the faces of the individual particles are beginning to form flat planes. The likely mechanism for this is surface transport, which is the primary mechanism for facet growth and formation ${ }^{23}$. After three days of exposure, faceting of the silver surface is evident but does not appear to be pronounced. The facets are short in nature, with some curvature visible, suggesting that the sample is in the early stages of faceting and that the formation of planes is not yet complete. 


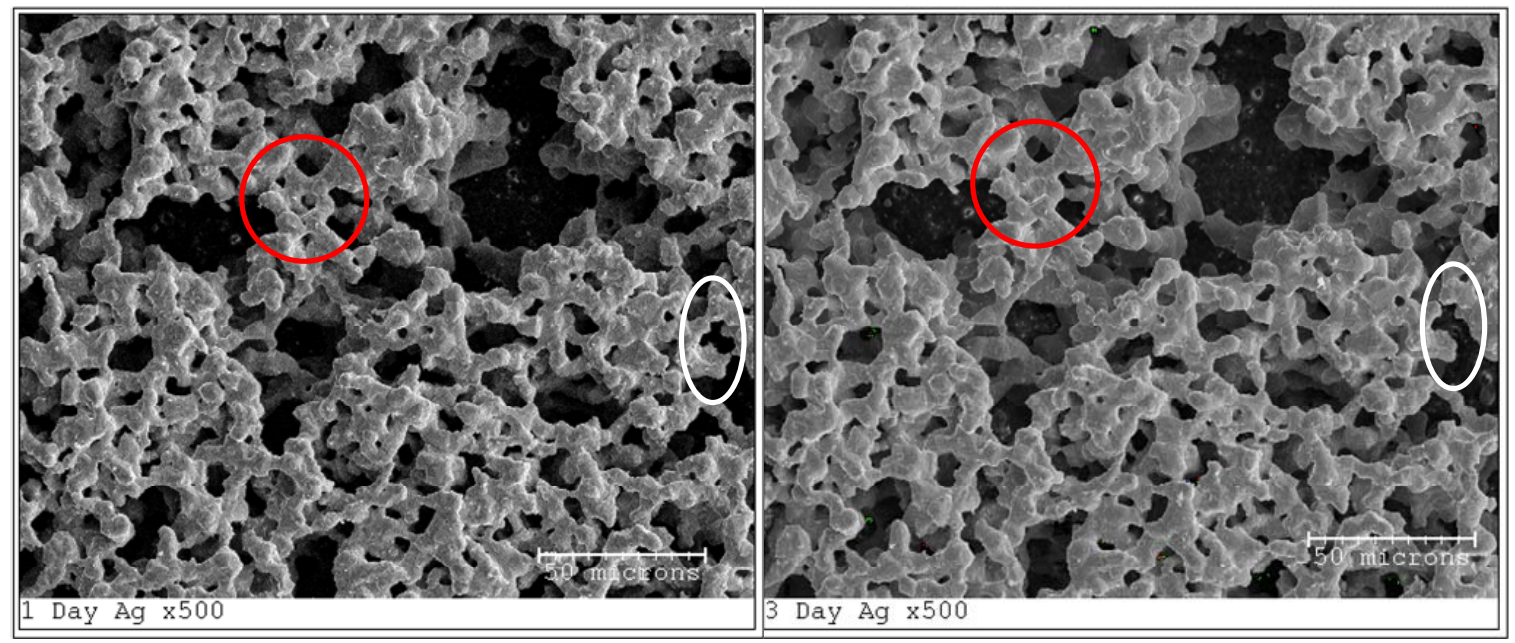

Figure 70 Silver surface comparison between day 1 and day 3

An evaluation of the same location on the printed surface between days one and three reveals that little has changed in terms of particle evaporation. There has been some noticeable evaporation and particle agglomeration, however to a minimal extent. The silver also experienced the loss of small particles that were exposed on multiple sides. While large scale mass loss was not clearly evident, small scale losses are visible. An example of particle agglomeration is circled in the figure above. The white oval shows necking that had occurred between two particles disappearing. This could be evidence of evaporation, but could also be the result of surface transport of molecules back into the larger particle, resulting in more agglomeration. Also apparent is the agglomeration of contiguous small particles into larger particles, most likely as a means of reducing the free surface energy of the sample. 


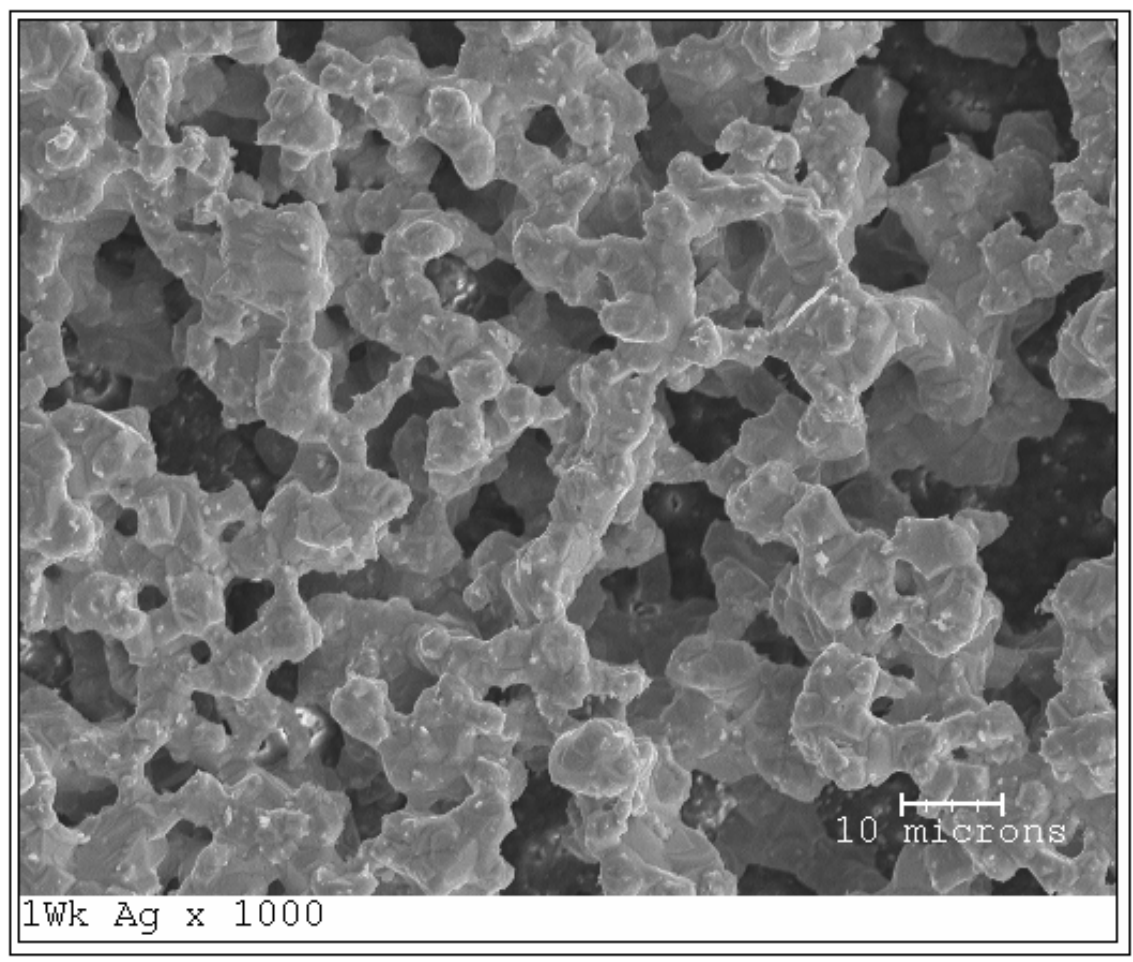

Figure 71 SEM micrograph of the silver surface (1 week exposure)

After one week of exposure it appears that little has changed in terms of surface structure of the sample. A micrograph of the surface after one week of exposure is shown in Figure 71. The appearance of the silver sample after one week of exposure to air flow at temperatures lower than the melting temperature of silver indicates that at $800{ }^{\circ} \mathrm{C}$ silver is stable enough to retain its general shape, but that particles evaporation and particle agglomeration occurs. 


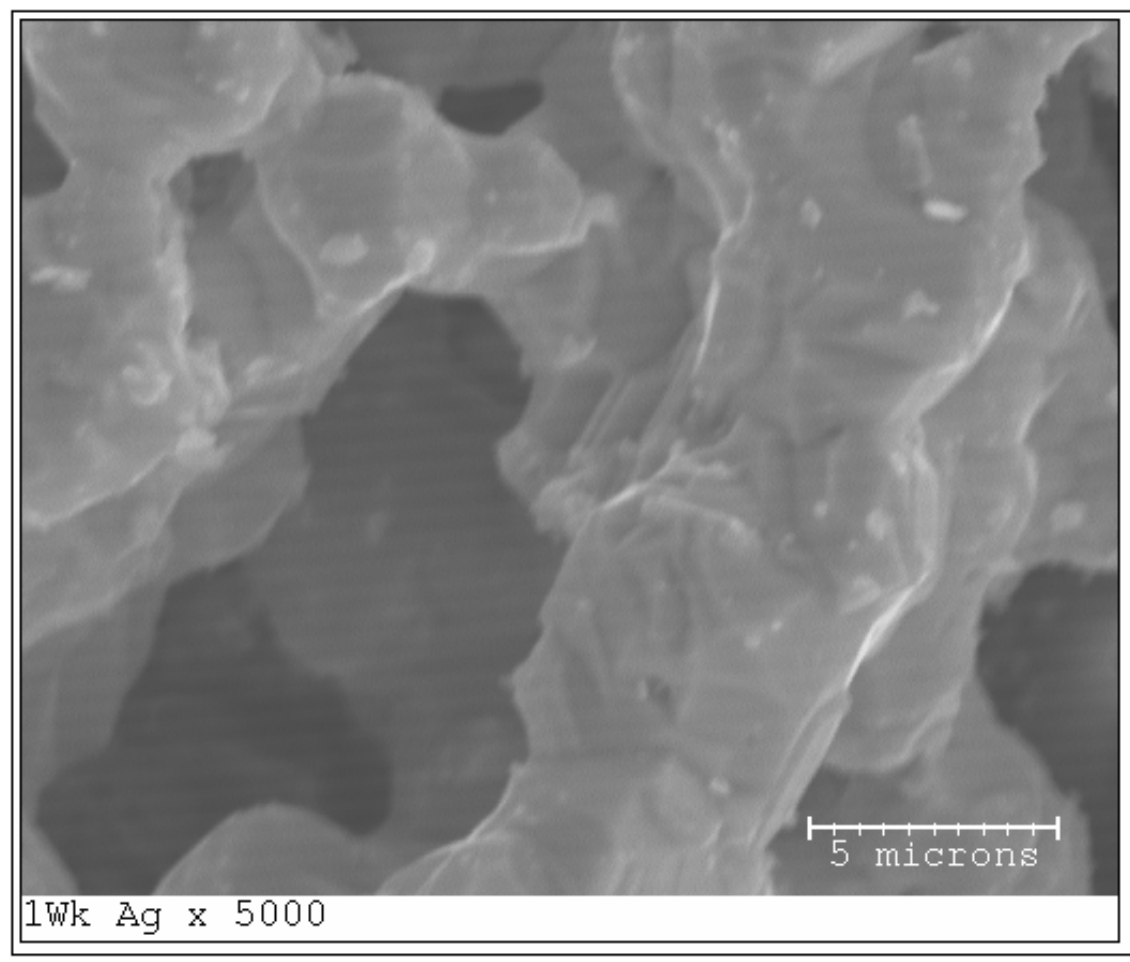

Figure 72 Printed silver after 1 week exposure showing more extensive faceting

After one week, faceting of the sample has taken place to a much greater extent on the faces of the particles. The forming of angular planes on the surface of each particle continues, resulting in a reduction in the curved facets that were visible after one and three days of exposure. 


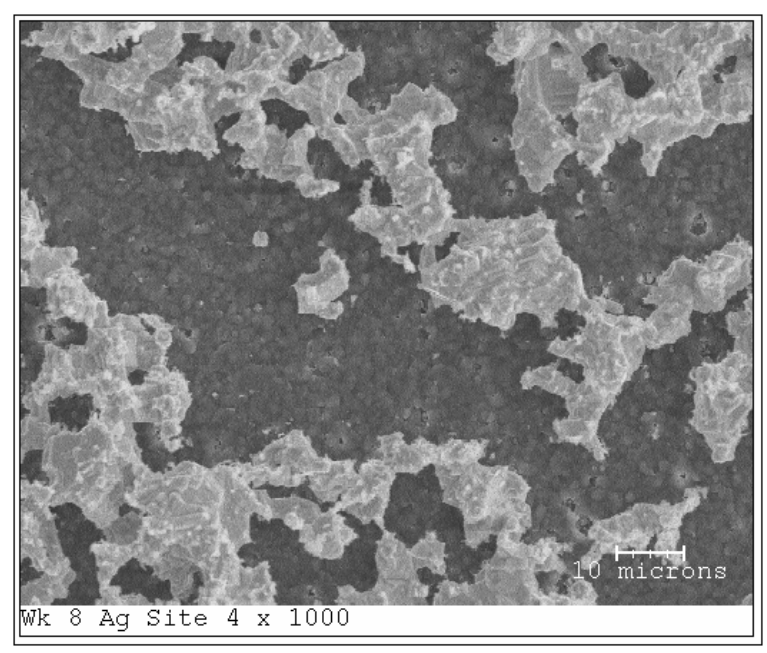

Figure 73 SEM micrograph of printed silver surface showing extensive particle loss (8 weeks exposure)

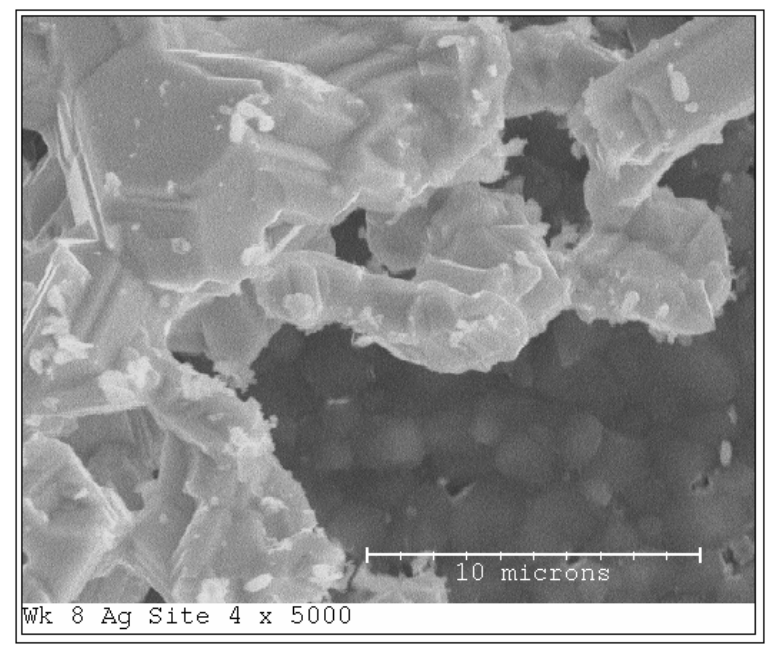

Figure 74 SEM micrograph of printed silver surface showing more extensive faceting (8 weeks exposure)

In the image taken during the eighth week of exposure, it is clear that the silver is evaporating. The gaps in the sponge-like morphology of the specimen have drastically increased in size as the silver has evaporated, which is reflected in the mass loss data. At this time the silver sample shows angular faceting over the entire surface. The facets are short in length and are not as dramatic as those that form on silver plate or foil after eight weeks. Almost all exposed surfaces of the particles consist of large faces or planes, with 
only one or two steps on each face. These steps are also larger than the ones that were visible on the sample after one week. Of note is the fact that the overall morphology of the sample retains the general structure of the original screen printed pattern. The particles are still clearly visible, having not coalesced into large particles and with the largest particles being approximately 6 microns, only slightly larger than after two weeks of exposure.

Over the course of the exposure time, the silver evaporated in a radial fashion. The radius of the circle of the screen printed silver remained relatively stable for the first 9 weeks, with only slight reduction at a linear rate. However, after 10 weeks of exposure, the sample experienced a sudden and rapid reduction in radius, such that the diameter of the sample was reduced from 0.5 " to approximately 0.20 ".

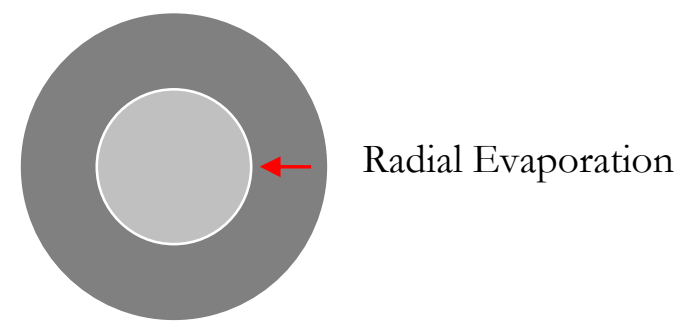

Figure 75 Description of evaporation of printed silver

This radical reduction in the radius of the sample occurred in a single week of exposure time and corroborates the samples behavior which was observed by the mass loss measurements. Also, the fact that after the sharp radial decrease, the sample retained its circular shape indicates that it was not due to particles blowing off. An unintended increase in flow rate would result in an oval shape. Also, other samples did not experience the same rapid mass loss, which discourages the idea of an unintended 
increase in temperature. The sharp loss in radius is therefore evidence of a non-linear evaporation rate.

Images taken after 16 weeks of exposure reveal more extensive faceting of the silver than the images taken after 8 weeks. While much of the silver has evaporated, the silver still maintains its general particulate morphology.

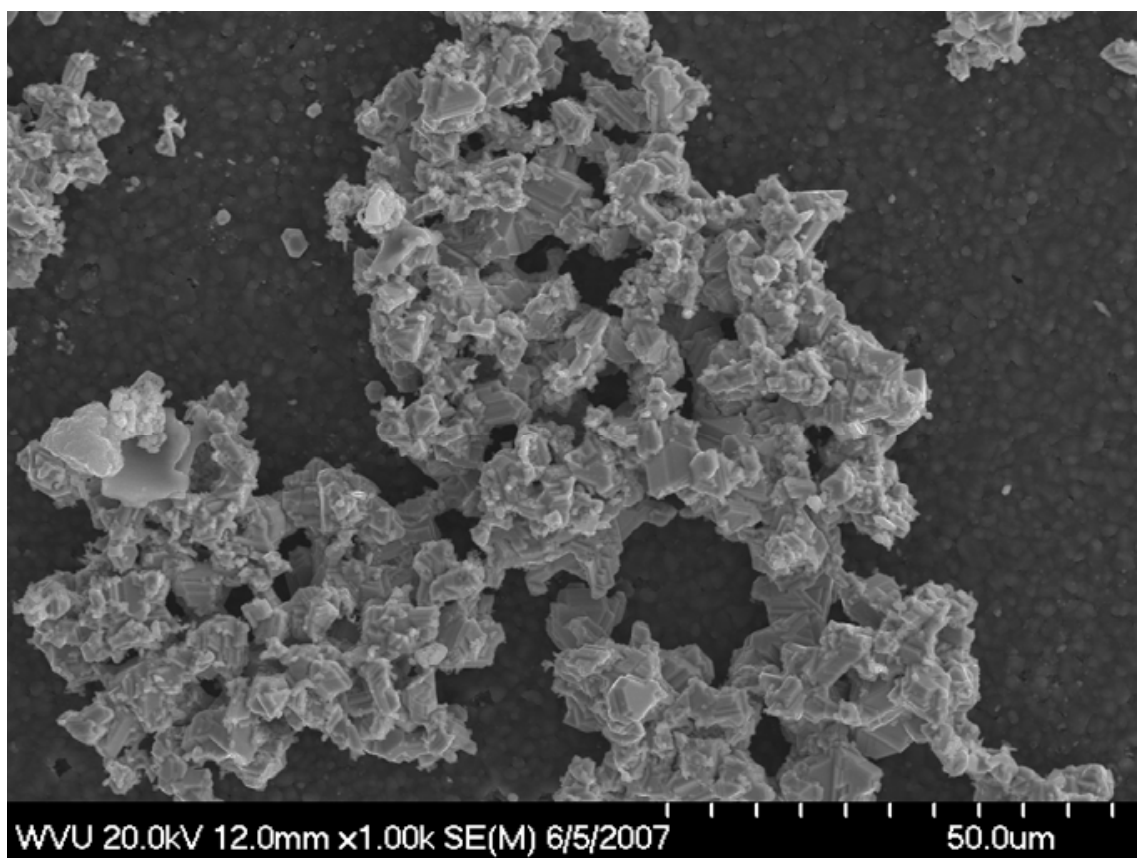

Figure 76 SEM micrograph of printed silver (16 week exposure) 


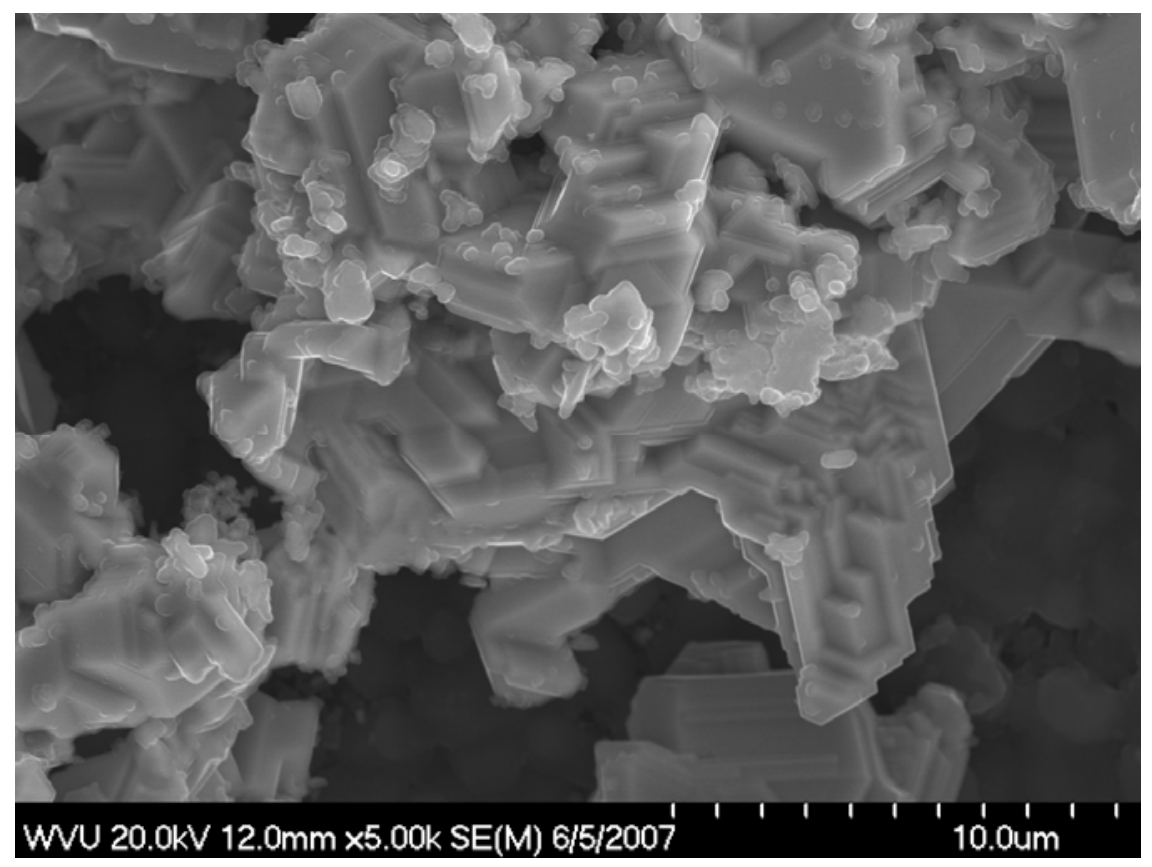

Figure 77 SEM micrograph of printed silver showing more extensive faceting (16 week exposure)

The faceting after 16 weeks is denser and more extensive across the surface. Most exposed planes of silver contain numerous facets, as opposed to the silver after 8 weeks, where many planes were unfaceted, or contained only one or two steps.

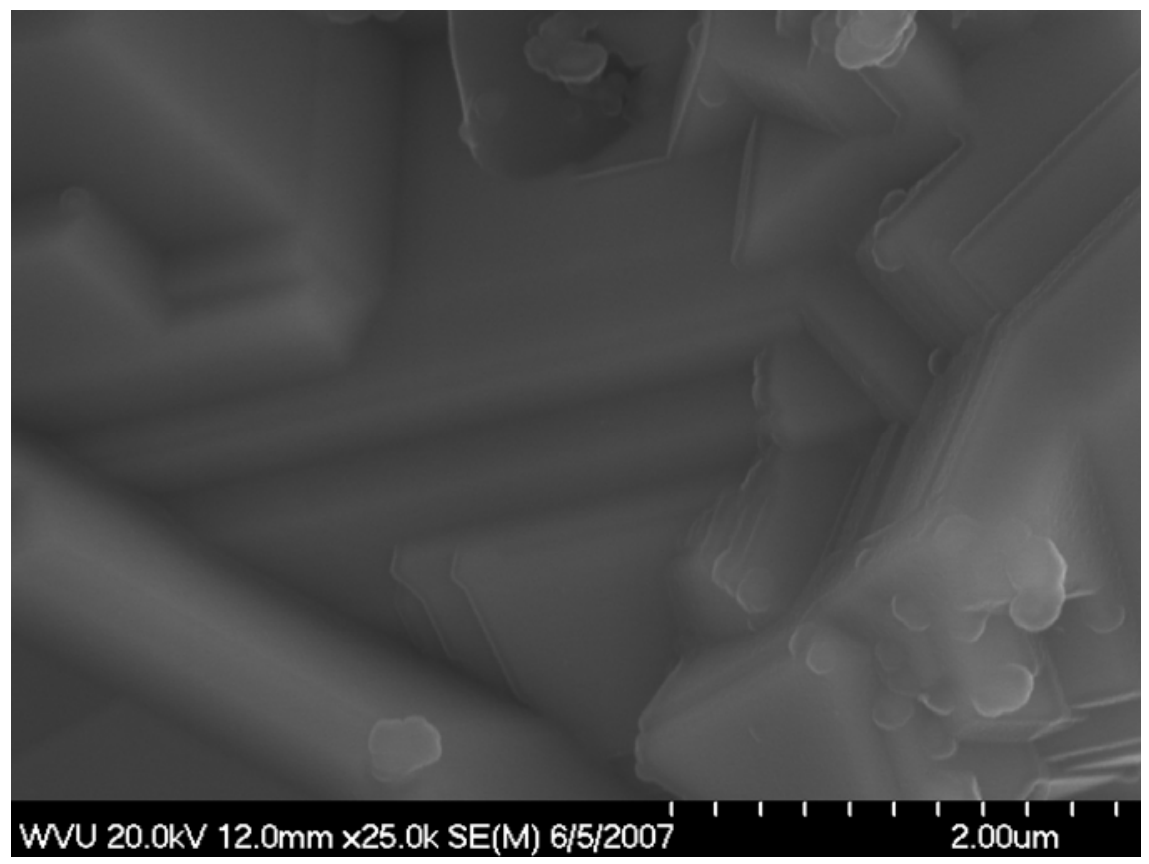

Figure 78 Evidence of small facets on surface of silver particles (16 week exposure) 
In the above micrograph very small facets are evident on the surface of the sample. Small facets are an indication of faceting that has yet to reach its most stable arrangement. This suggests that after 16 weeks, the silver surface is still undergoing changes to its surface structure in an attempt to reach a stable crystalline arrangement.

\subsubsection{Pure Silver Foil Sample}

Samples of 50 micron thick silver foil were exposed to the same conditions as the other samples. These samples were made from a pure silver foil that was purchased and cut to size. The dimensions and masses of the sample were measured prior to exposure. The surfaces of the samples were cleaned with acetone. Samples were imaged weekly using SEM to serve as a method of evaluating the faceting behavior of the screen printed samples.

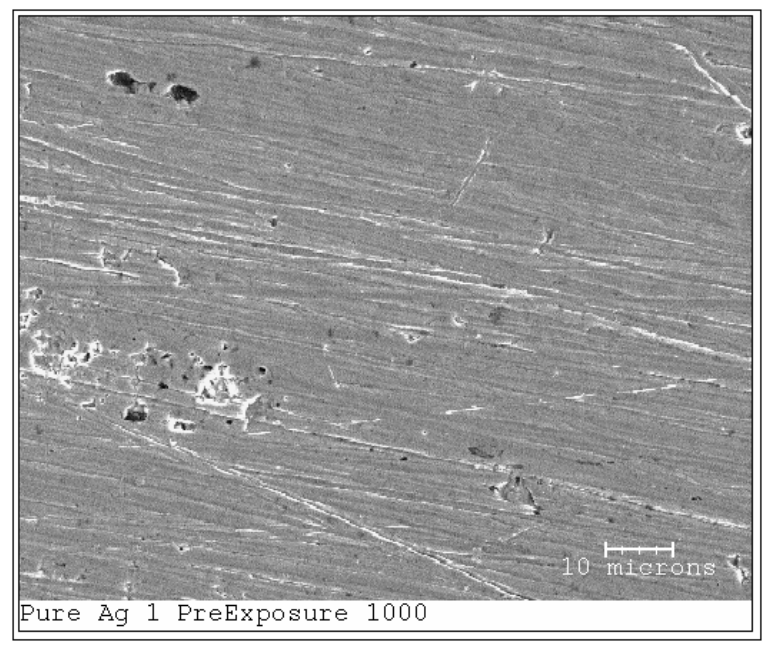

Figure 79 SEM micrograph of pre exposure silver foil surface 
There are no visible features on the surface of the pre-exposure sample. The sample was documented to be pure silver using X-ray analysis.

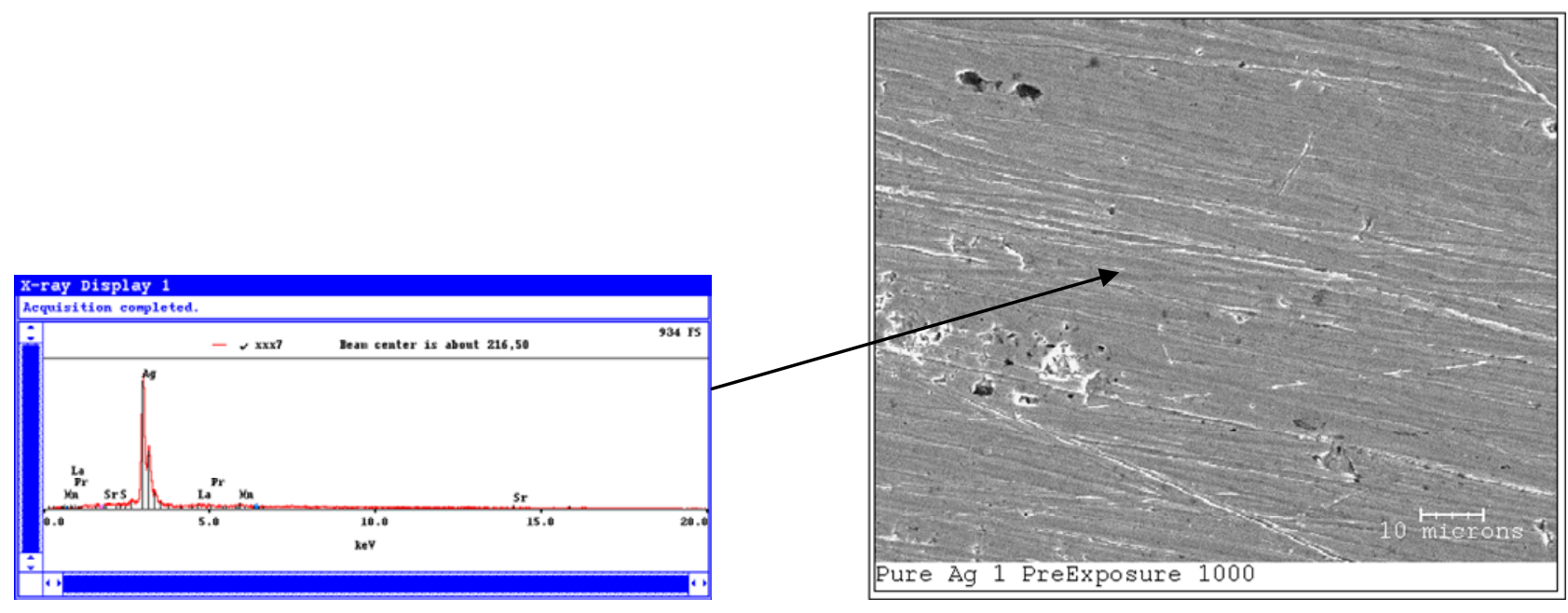

Figure $80 \mathrm{X}$-ray spot analysis of pre exposure silver foil surface

The surface of the pure silver shows significant faceting after just one week of exposure.

This is in significant contrast to the screen printed nanoparticles, which experienced faceting after two weeks, but in a far less extensive manner.
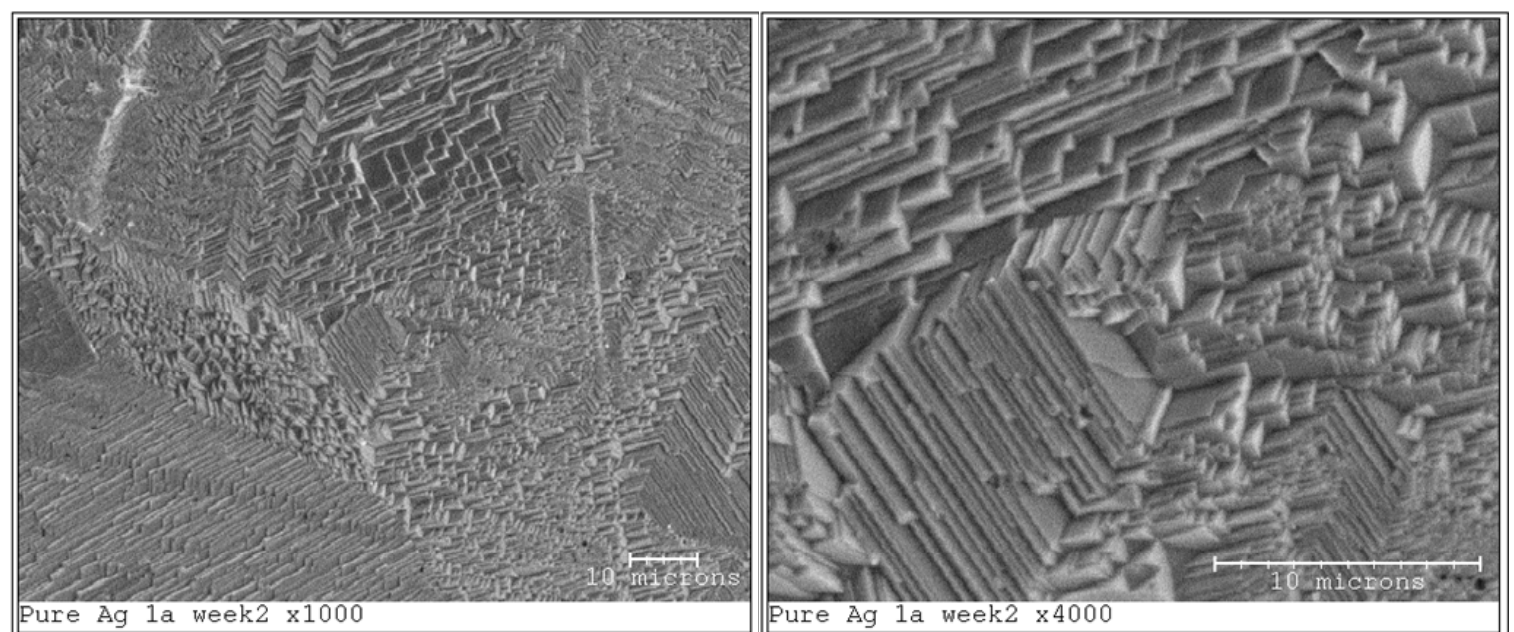

Figure 81 SEM micrograph of silver foil surface after 1 week exposure at $1 \mathrm{k}$ and $4 \mathrm{k}$ magnification 
Changes to the surface of the pure silver sample are documented in the following series of micrographs ${ }^{14}$. 


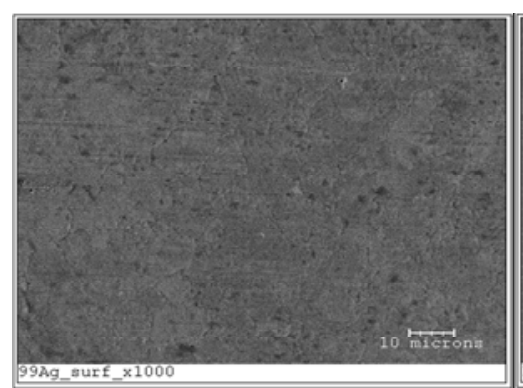

Pre-Exposure

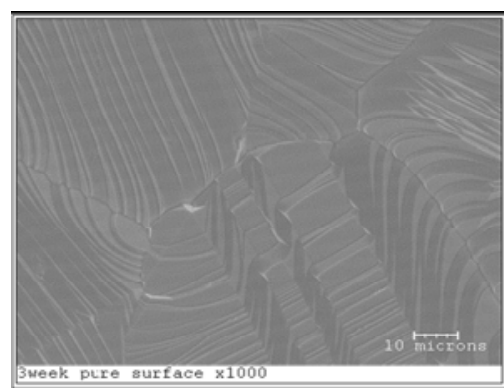

Week 3

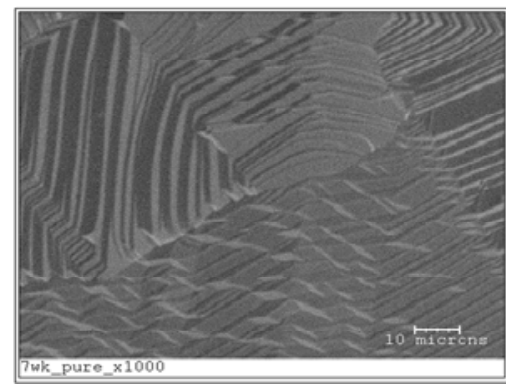

Week 7

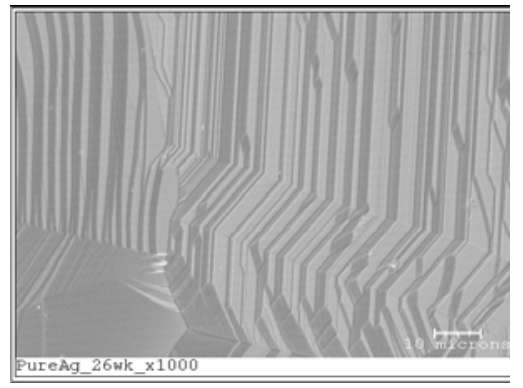

Week 26

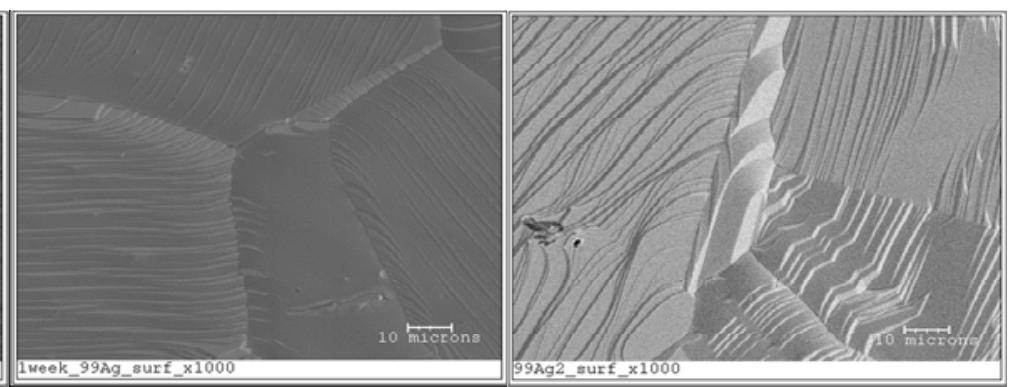

Week 1

Week 2

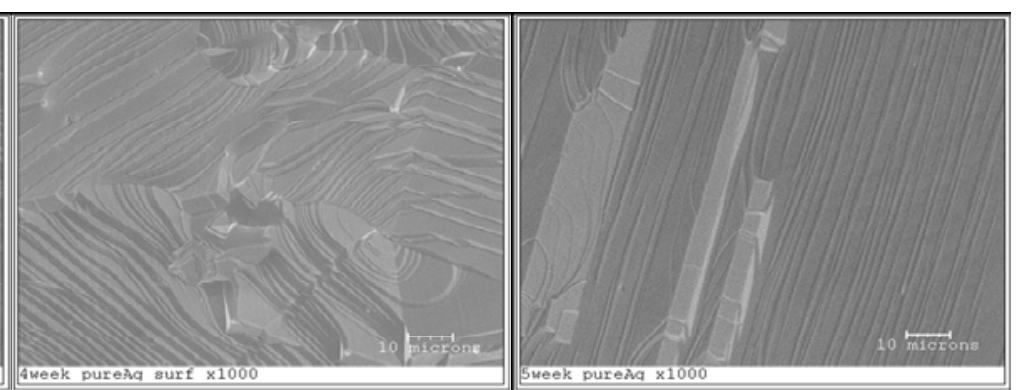

Week 5

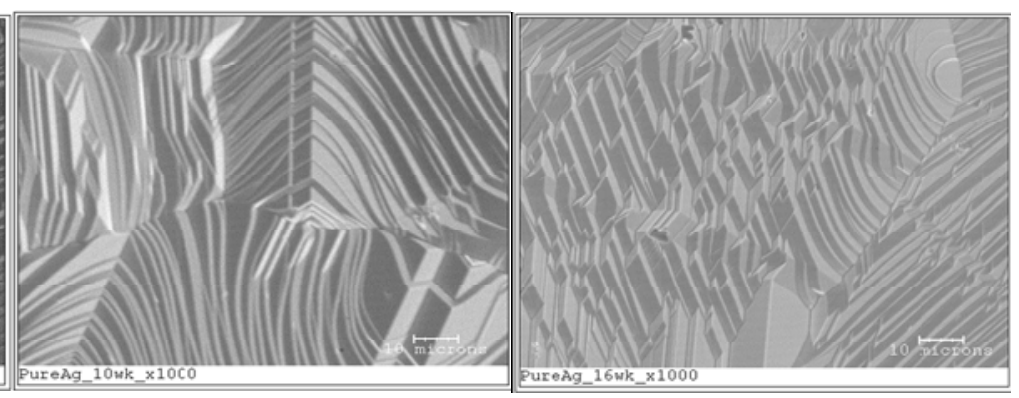

Week 10

Week 16

Figure 82 Pure silver surfaces after high temperature exposure

It is clear from the above images that after one week, the surface of the sample experiences microstructure changes only in the form of further faceting. The faceting that 
is visible after one week of exposure follows the grain structure of the foil sample, with the direction of the faceting changing at grain boundaries ${ }^{8}$. As the exposure time continues, the faceting of the surface becomes much more pronounced. The silver foil is able to form much larger facets over its surface area than the silver powder, as the cured particulate nature of the silver powder hinders the growth of larger facets that would provide thermal evaporative stability. 


\section{CHAPTER 5 DISCUSSION AND CONCLUSION}

\subsection{DISCUSSION AND CONCLUSION}

The purpose of this research was to prove that the use of a protective coating layer over screen printed silver would inhibit the evaporative loss of silver during high temperature exposure. The data collected during the course of the experiment indicates that this does in fact occur, as the coated samples have a lower mass loss rate than the uncoated samples and a lower rate than silver foil.

In was discovered that silver may have a non-linear evaporation rate, however a study using a greater number of samples would have to be conducted to determine this conclusively. Generally, silver samples lose mass at a similar, roughly uniform rate each week. However, it was noted that periods of rapid mass loss seemed to occur during the evaporation study. These periods were evidenced by the non-linear evaporation of the silver and occurred at varying times, seemingly dependent upon the composition of the sample. This dependence included whether the sample was coated with LSM or the percentage of ceria. Screen printed silver particles have the longest exposure period before non-linear evaporation occurs, which happened after twelve weeks. The silverLSM sample had the next longest exposure period before increase mass loss occured, with the non-linear evaporation occurring at the eleventh week. The LSM coated silverceria composite samples of $10 \%$ and $40 \%$ ceria had their period of rapid evaporation occur at nine and three weeks, respectively. It seems that while the use of an LSM coating layer reduces the evaporation rate of silver, it hastens the time at which non- 
linear evaporation occurs. Similarly, the addition of ceria appears to hasten the non-linear evaporation time as well.

The apparent non-linear evaporation rate of silver was noticed in the data for 50 micron silver foil, occurring after both six and eleven weeks. This provides evidence that the non-linear evaporation is not caused by any systemic problems or short term perturbation in the experiment parameters. This phenomenon is likely caused by the rearranging of the surface orientation of the silver, and is linked to the formation of facets $^{14}$.

The printed silver experiences bulk diffusion into the LSM coating layer, which begins to occur immediately upon exposure. This diffusion occurs over the entire exposure period and results in a cavity within the sample layering where the silver contact paste was originally supposed to be. Diffusion continues to occur until all silver has diffused from the deposition area into the LSM layer. The time period during which silver diffuses completely from the deposition location is believed to occur in the weeks before the non-linear mass loss, meaning that for the silver-LSM, silver/10\%ceria-LSM and silver $/ 40 \%$ ceria-LSM samples, all silver will have diffused into the LSM layer between weeks 4 and 10, 3 and 7, and 2 and 3, respectively. Cross section analysis of a silver-LSM sample indicated that some silver is still largely in the original deposition location after 2 weeks of exposure, but that diffusion had begun.

The region of maximum concentration of silver within the LSM layer was determined to move closer to the free surface of the sample each week. Most likely, the mechanism driving silver diffusion through the LSM layer is bulk transport. Fracture surface X-ray analysis reveals that as exposure time increases, silver migrates at higher 
rates into the LSM. Fracture surface analysis also showed that the use of ceria did not serve as an anchor for the silver. Contrary to what was expected, increasing percentages of ceria resulted in an increased diffusion rate of silver into the LSM layer. Mass loss studies concluded that the use of a bimetal composite of ceria and silver actually increased the mass loss rates of the silver in the sample.

It is apparent that the LSM coating layer has excellent thermal stability. The introduction of ceria into the sample is not believed to have had a causative effect with regard to cracking due to thermal expansion mismatch as no increase in the cracking of the surface of the samples containing ceria was noticed over those samples containing only silver. Cracking did occur to a similar extent on the LSM of the silver-LSM samples as it occurred on the samples containing ceria.

It is possible that the diffusion of silver into the LSM cathode could inhibit oxygen permeation of the cathode, although the conditioning time and diffusion of silver found by Simner could indicate that silver diffusion has a beneficial effect upon cell operation. Oxygen permeability of dense silver increases with temperature ${ }^{24}$, so it is possible that silver diffusion into the cathode would improve oxygen ion transport to the anode. It is also possible that due to an increase in density at the cathode electrolyte interface, oxygen transfer to the electrolyte could be hindered by silver diffusion.

\subsection{CONCLUSION}

Data collected in this investigation indicates that a layer of screen printed, submicron sized silver particles, covered by a layer of printed LSM, evaporate at a rate 
roughly half that of screen printed silver and roughly one-tenth the rate of the 50 micron thick silver foil determined by Sakacsi. While it was originally thought that the inclusion of ceria as a bimetal composite in the silver would reduce the evaporation rate of the silver, the $10 \%$ and $40 \%$ ceria samples had mass loss rates three and four times greater than the silver-LSM samples, respectively.

Silver diffusion into a pervoskite membrane, such as the LSM coating of the contact paste samples in this study would increase the density of the cathode layer. The diffusivity of the cathode could possibly be affected by this, and could restrict air access to the electrolyte. This could result in a degradation of the power density of the cell. Also, the silver diffusion noticed in this study completely defeats to original purpose of this study, which was to determine the possibility of silvers use as a contact material between the cathode and interconnect. If silver diffuses into the cathode material, then no contact material will remain to serve as a current path.

The diffusion of the screen printed silver into the LSM layer is possibly due to the higher general free surface energy of the printed sample. Evidence from the surface analysis of LSM indicates that LSM is highly stable at high temperature. Potential still remains for the use of LSM as a protective evaporative coating of silver foil, which would have a lower surface energy than the printed samples and could potentially have a lower diffusion rate as the surface exchange rate could be lowered by the reduction in interfaces between the LSM and silver foil. The use of diffusion barriers, such as thin, dense layers of manganese or cobalt, could be used to prevent silver diffusion from the contact region. Also, the coating of the free surface of the LSM layer with an electrolyte 
could help determine the mechanism behind silver diffusion and if the bulk diffusion of silver is tied to any evaporative causes at the free surface 


\section{REFERENCES}

1. W.Z. Zhu, S.C. Deevi; Develompent of Interconnect Materials for Solid Oxide Fuel Cells; Materials Science and Engineering A348 (2003) 227-243.

2. James Larminie and Andrew Dicks; Fuel Cell Systems Explained; 2nd Edition 2003.

3. Willem Joseph Quaddakkers, Javier Pirón-Abellán, and Vladimir Shemetl; Metallic Materials in Solid Oxide Fuel Cells; Forschungszentrum Julich. Institute for Materials and Processes in Energy Systems 52425 Julich, FRG.

4. S.C. Singhal, Science and Technology of Solid-Oxide Fuel Cells.

5. San Ping Jiang, Siew Hwa Chan; A review of anode materials development in solid-oxide fuel cells; Fuel Cells Strategic Research Program, School of Mechanical and Production Engineering, Nanyang Technological University; Journal of Material Sciences 39 (2004) 4405-4439.

6. Zongping Shao, Sossina M. Haile; A High-Performance Cathod for the Next Generation of Solid- Oxide Fuel Cells; Materials Science, California Institute of Technology, Pasadena, California 91125 USA.

7. W.A Meulenburg, O. Teller, U. Flesch, H.P. Buchkremer, and D. Stover; Improved Contacting by the use of Silver in Solid Oxide Fuel Cells up to an Operating Temperature of $800^{\circ} \mathrm{C}$; Journal of Materials Sciences 36 (2001) 31893195.

8. B. Chalmers, R. King, R. Shuttleworth; The thermal etching of silver; Royal Aircraft Establishment, South Farnborough, Hants (1948). 
9. Zhanguo Yang, Guanguang Xia, Jeffrey Stevenson; Electrical Contacts Between Cathodes and Metallic Interconnects in Solid-Oxide Fuel Cells; Pacific Northwest National Laboratory, 902 Battelle Blvd., Richland, WA 99352.

10. Maria Flytzani-Stephanopoulos; Nanostructured cerium oxide ecocatalysts; MRS Bulletin, November 2001.

11. Zhao Sufang; Study of the catalytic properties of ceria-based materials in the WGS reaction and hydrocarbon oxidations, University of Pennsylvania. 2005. Dissertation.

12. A.L. Barg, E. Rabkin, and W. Gust; Faceting transformation and energy of a $\Sigma 3$ grain boundary in silver; Acta metal. Mater. 43 no 11 (1995) 4067-4074.

13. Abhishek Jaiswal, Eric Wachsman; Fabrication of Anode Supported Thick Film Ceria Electrolytes for IT-SOFC's; Journal of Ionics, 11, 3-4 May 2005.

14. John Sakacsi; Evaluation of Sterling Silver as a Contacting Material for the Cathode Chamber of the Solid-Oxide Fuel Cell; Dept. of Mechanical and Aerospace Engineering, West Virginia University (2006).

15. S.P Simner, Michael D. Anderson, James Coleman, Jeffry Stevenson; Performance of a novel $\mathrm{La}(\mathrm{Sr}) \mathrm{Fe}(\mathrm{Co}) \mathrm{O}_{3}-\mathrm{Ag}$ SOFC Cathode; J. of Power Sources 161 (2006) 115-122.

16. S.P Simner, Michael D. Anderson, Jared W. Templeton, Jeffry Stevenson; $\underline{\text { Silver- }}$ perovskite composite SOFC cathodes processed via mechanofusion; J of Power Sources 168 (2007) 236-239. 
17. K. Zhang, Y.L. Yang, D. Ponnusamy, A.J. Jacobson, K. Salama; Effect of microstructure on oxygen permeation in $\mathrm{SrCO}_{0.8} \underline{\mathrm{Fe}_{0.2}} \underline{\mathrm{O}_{3-\delta}} ; \mathrm{J}$. of Materials Science 34 (1999) 1367-1372.

18. J. Bernardini, C.H. Cirardeaux, and A. Rolland; Effect of grain boundary segregation and migration on diffusion profile: analysis and experiments; Interface Science 11 (2003) 33-40.

19. Marian Chatenet, Marc Aurousseau, Robert Durand, Francoise Andolfatto; $\underline{\text { Silver- }}$ platinum bimetallic catalysts for oxygen cathodes in chlor-alkali electrolysis; J. of the Electrochemical Society 150 (2003) D47-D55.

20. B.A. McCool, Y.S. Lin; Nanostructured thin palladium-silver membranes: Effects of grain size on gas permeation properties; J of Material Science 36 (2001) 32213227.

21. Jae Bon Koo, Duk Yong Yoon; The dependence of normal and abnormal grain growth in silver on annealing temperature and atmosphere; Metallurgical and Materials Trans. 32A (2001) 469-475.

22. Randall German; Powder Metallurgy Science; Metal Powder Industries Federation, 1984

23. Ying Liu, Charles Compson, Meilin Liu Nanostructured and functionally graded cathodes for intermediate temperature solid oxide fuel cells; J. of Power Sciences 1238 (2004) 194-198.

24. YongMan Choi and Meilin Liu; Functionally Graded Cathodes for Solid Oxide $\underline{\text { Fuel Cells; }}$; Center for Innovative Fuel Cell and Battery Technologies, Georgia Tech (2006). 
25. Kwang Bo Shim, Nam Tae Cho and Seon Woo Lee; Silver diffusion and microstructure in LTCC multilayer couplers for high frequency applications; J. of Materials Science 35 (2000) 813-820.

26. John C. Vickerman; Surface Analysis The Principal Techniques; Wiley (2006). 